Marisa Schmidt Bazzi

\title{
Breakup dynamics of non-Newtonian thin \\ liquid sheets
}

Dissertation presented to the Programa de Pós-Graduação em Engenharia Mecânica of PUC-Rio in partial fulfillment of the requirements for the degree of Mestre em Engenharia Mecânica.

Advisor: Prof. Márcio da Silveira Carvalho 


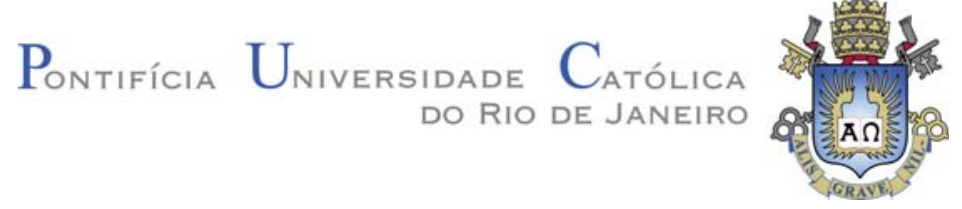

Marisa Schmidt Bazzi

\title{
Breakup dynamics of non-Newtonian thin \\ liquid sheets
}

Dissertation presented to the Programa de Pós-Graduação em Engenharia Mecânica of PUC-Rio in partial fulfillment of the requirements for the degree of Mestre em Engenharia Mecânica. Approved by the undersigned Examination Committee.

\author{
Prof. Márcio da Silveira Carvalho \\ Advisor \\ Departamento de Engenharia Mecânica - PUC-Rio \\ Prof. André Nachbin \\ Instituto de Matemática Pura e Aplicada - IMPA \\ Prof. Igor Braga de Paula \\ Departamento de Engenharia Mecânica - PUC-Rio
}

Prof. Márcio da Silveira Carvalho

Vice Dean of Graduate Studies

Centro Técnico Científico - PUC-Rio

Rio de Janeiro, April 11th, 2018 
All rights reserved.

Marisa Schmidt Bazzi

Bachelor in Engenharia Mecânica by Pontifícia Universidade Católica do Rio de Janeiro (2015)

Bibliographic data

Schmidt Bazzi, Marisa

Breakup dynamics of non-Newtonian thin liquid sheets / Marisa Schmidt Bazzi; advisor: Márcio da Silveira Carvalho. - Rio de janeiro: PUC-Rio, Departamento de Engenharia Mecânica, 2018.

v., 109 f: il. color. ; $30 \mathrm{~cm}$

Dissertação (mestrado) - Pontifícia Universidade Católica do Rio de Janeiro, Departamento de Engenharia Mecânica.

Inclui bibliografia

1. Engenharia Mecânica - Teses. 2. Modelo OldroydB;. 3. Análise de estabilidade;. 4. Simulação Numérica;. 5. Quebra de filme de liquido.. I. da Silveira Carvalho, Márcio. II. Pontifícia Universidade Católica do Rio de Janeiro. Departamento de Engenharia Mecânica. III. Título. 
To my parents and my siblings, for their support and encouragement. 


\section{Acknowledgments}

First, I would like to thank my parents for their unconditional love, for always supporting my decisions and goals, despite all the difficulties that life has imposed on them. I would like to express my deepest gratitude to them, who, with great effort and untiring dedication, have turned our house into a home, providing a great life for our family. To them, that in their simplicity taught me to fight for my goals and never give up, even when the path is not easy. Thank you for giving me life and for teaching me all the values I bring in my heart. I love you so much!

I would like to thank my old sister, Marilise Bazzi, for all her support and love, for practically being my second mother, for giving me all the assistance that I needed when I moved to Rio de Janeiro, without you none of this would be possible. My twin sister, Mariéli Bazzi, for being by my side since from the belly of our mother, for being the best friend and life partner I could have. To my brother, Mauro Bazzi, for the care and love with our parents, when we could not be present. My niece Julia Pimenta, for the incredible person that she is, for the immense care and love that she has with me. Love you all!

To my boyfriend Rafael Silva, for your care, love, and mainly for the patience and understanding during this time. Thank you for being the best word, the best shoulder, and the best hug, in my worst days. For being such an amazing and inspiring person and always encouraging me to be better. For all the deep conversations and lessons about history, politics and life. In short, thank you for being so special!

To my advisor, Prof. Marcio Carvalho, for the great opportunity to work in his group, for the magisterial classes, for the trips to conferences and for the extensive discussions, not only about academic subjects, but about many different topics. Thank you for not only guiding me but inspiring me as a professional and person.

To Nicolle Lima, for the great friendship and affection that we cultivated during these two years, for the trips and for the excellent moments that we had together. Thank you for being always available to listen to me, to support me and to advise me. I just have to thank God for including you in my path.

To Débora Nascimento for being the best footvolley partner, for always being so friendly and for listen to me and give me the best tips and advice. To Frederico Gomes, for being my lifesaver in the most different subjects.

To my friend João Virgolino, for sharing with me the best and worst experiences of master's studies, and to be always by my side, listening to me and supporting me. 
To all my fellow friends from the LMMP group, in particular, Talita, Paulo, Paula, Marcelo, Rafael, Monique, Sabrina, Gabriel, and Mariano, for the good times, laughs and knowledge shared during this two years.

In short, this work is the result of efforts and contributions, not only of the people who have been by my side in the last two years but of all those who directly or indirectly contributed to my professional, academic and personal development. Surely everybody was essential in building the person I am today. Thank you all so much.

Finally, to the National Council of Scientific and Technological Development $(\mathrm{CNPq})$ and Foundation for Research Support of the State of Rio de Janeiro (FAPERJ) for the scholarship and to all Brazilians that invest, through your taxes, in the promotion of serious research in many different fields. 


\section{Abstract}

Schmidt Bazzi, Marisa; da Silveira Carvalho, Márcio (Advisor). Breakup dynamics of non-Newtonian thin liquid sheets. Rio de Janeiro, 2018. 109p. Dissertação de Mestrado - Departamento de Engenharia Mecânica, Pontifícia Universidade Católica do Rio de Janeiro.

Thin free liquid sheets are ubiquitous in many industrial processes, such as atomization and curtain coating. Liquid sheets are susceptible to instabilities at the interface, which can grow, triggering a breakup process. This process can be divided into two different stages: the rupture stage and retraction. The first, driven by van der Waals force, occurs when a small instability grows until it pinches-off the sheet. The second, driven by capillary forces, induces the growth of the hole caused by the pinch-off, leading to the full disintegration of the liquid sheet. The stability of a liquid sheet depends on disturbance characteristics, sheet thickness, and fluid properties. Experimental analyses have shown that thinner stable liquid curtain can be obtained with viscoelastic liquids. The underlyning physical mechanisms associated with increased stability are, however, not fully understood. This work presents a theoretical and numerical analysis of the effect of viscoelasticity on the stability of a thin liquid sheet during both stages of the breakup process. We first analyze the rupture dynamics, deriving linear stability criteria for both planar and axisymmetric perturbations of Newtonian and Oldroyd-B liquids. The time evolution of planar and axisymmetric perturbations in an Oldroyd-B liquid sheet is evaluated using the asymptotic expansion of the flow variables and a fully-implicit time integration scheme. The rupture time and retraction velocity are calculated as a function of the viscoelastic properties. The results show that the liquid rheological behavior does not influence the linear stability criterion. Nevertheless, it has a strong effect on the growth rate of the disturbance and retraction velocity, increasing, thus, the breakup time. The results show that elastic forces act to hinder the rupture and retraction stages. Analysis of the temporal evolution of the thickness profile reveals that liquid rheological behavior also affects the shape of the liquid sheet. For low viscosity regime, the elastic forces damp the capillary waves that arise during the retraction of Newtonian sheets.

\section{Keywords}

Oldroyd-B model; Stability analysis; Numerical Simulation; Liquid sheet breakup. 


\section{Resumo}

Schmidt Bazzi, Marisa; da Silveira Carvalho, Márcio. Dinâmica da quebra de filmes finos não Newtonianos. Rio de Janeiro, 2018. 109p. Dissertação de Mestrado - Departamento de Engenharia Mecânica, Pontifícia Universidade Católica do Rio de Janeiro.

Filmes finos de líquidos estão presentes em uma gama de aplicações industriais, como processos de atomização e revestimento de substrato. $\mathrm{O}$ processo de quebra pode ser divido em duas etapas: o estágio de ruptura, e o estágio de retração. O primeiro, movido pelas forças de van der Waals, ocorre quando uma pequena perturbação cresce e provoca o aparecimento de um pequeno furo no filme. O segundo, movido por forças capilares, provoca o crescimento desse furo levando à desintegração do filme de líquido. A estabilidade de uma cortina de líquido depende das características da perturbação, da espessura do filme e das propriedades do fluido. Análises experimentais mostraram que uma cortina super fina pode ser obtida pela utilização de fluidos viscoelásticos. Os mecanismos físicos associados à esta estabilidade, contudo, não são totalmente compreendidos. Este trabalho apresenta um estudo numérico e teórico dos efeitos das propriedades viscoelásticas na estabilidade de uma cortina de fluido, englobando ambos os estágio do processo. As análises numéricas foram desenvolvidas através da expansão assintótica das variáveis do escoamento com aplicação de um esquema de integração no tempo totalmente implícito. A partir da análise teórica da dinâmica de ruptura foi possível obter um critério de estabilidade linear para perturbações planares e axissimétricas em fluidos Newtonianos e não-Newtonianos. O tempo de ruptura e a velocidade de retração do filme foram calculados numericamente como função das propriedades viscoelásticas do líquido. Resultados mostraram que as forças elásticas atuam de forma a dificultar o processo de quebra e retração. Análises da evolução da espessura mostraram que as propriedades reológicas do fluído também interferem no formato que o filme de fluido assume durante o processo de retração. Para regimes de baixa viscosidade, as forças elásticas atuaram evitando a formação de ondas capilares observadas em fluidos Newtonianos.

\section{Palavras-chave}

Modelo Oldroyd-B; Análise de estabilidade; Simulação Numérica; Quebra de filme de liquido. 


\section{Table of contents}

1 Introduction $\quad 16$

$\begin{array}{ll}1.1 \text { Motivation } & 16\end{array}$

$\begin{array}{ll}1.2 & \text { Literature review } \\ 1.217\end{array}$

$\begin{array}{lll}1.2 .1 & \text { Sheet rupture } & 17\end{array}$

1.2.2 Sheet retraction 21

$\begin{array}{lll}1.3 & \text { Dissertation goals } & 25\end{array}$

1.4 Scope of work 26

I Rupture process $\quad 27$

2 Mathematical modeling $\quad 28$

2.1 Conservations equations 28

2.1.1 Generalized Newtonian model 30

2.1.2 Oldroyd-B model 31

2.2 Boundary conditions 33

2.3 Lubrication approximation 35

2.3.1 Planar model 36

2.3.2 Axisymmetric model 39

3 Linear stability $\quad 42$

3.1 Planar model $\quad 42$

3.2 Axisymmetric model $\quad 45$

4 Solution method $\quad 46$

4.1 Finite difference method 46

4.2 Crack-Nicholson method 49

4.3 Newton's method 50

4.3.1 Validation 51

$5 \quad$ Results and discussion $\quad 53$

5.1 Numerical determination of stability criterion $\quad 53$

5.2 Effect of non-Newtonian properties $\quad 56$

5.2.1 Oldroyd-B model $\quad 56$

5.3 Similarity analysis $\quad 63$

5.3.1 Generalized Newtonian model 65

$\begin{array}{ll}\text { II Sheet retraction } & 70\end{array}$

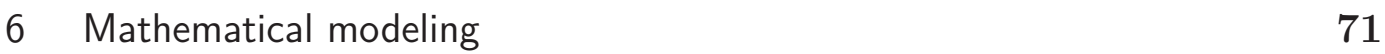

$\begin{array}{lll}6.1 & \text { Conservations equations } & 71\end{array}$

$\begin{array}{lll}6.2 & \text { Boundary condition } & 72\end{array}$

$\begin{array}{lll}6.3 & \text { Initial condition } & 74\end{array}$

$\begin{array}{ll}6.4 & \text { Lubrification approximation } \\ \end{array}$ 
6.4.1 Straight line retraction $\quad 75$

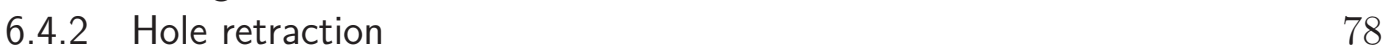

7 Solution method $\quad 81$

7.1 Velocity tip condition $\quad 81$

7.1.1 Straight line tip velocity $\quad 82$

7.1.2 Hole tip velocity 83

7.2 Curvature singularity problem $\quad 84$

$\begin{array}{ll}\text { 7.3 Mapping domain transformation } & 85\end{array}$

$\begin{array}{lll}7.4 & \text { Initial thickness profile } & 88\end{array}$

$\begin{array}{lll}7.5 & \text { Finite difference method } & 88\end{array}$

$\begin{array}{lll}7.6 & \text { Numerical validation } & 89\end{array}$

8 Results and discussions $\quad 91$

8.1 High Oh regime $\quad 91$

8.1.1 Oldroyd-B model $\quad 91$

8.1.2 Generalized Newtonian model 94

8.2 Low Oh regime 96

$\begin{array}{ll}\text { 8.2.1 Oldroyd-B model } & 97\end{array}$

9 Conclusions 103

$\begin{array}{lll}9.1 & \text { Future works } & 104\end{array}$

$\begin{array}{ll}\text { Bibliography } & 105\end{array}$

$\begin{array}{ll}\text { A Published paper } & 109\end{array}$ 


\section{List of figures}

Figure 1.1 Desintegration process in a Newtonian liquid sheet caused by the growth of a small role due to action of surface tension [17].

Figure 2.1 Sketch of the rheological behavior of Generalized Newtonian model under an extensional flow.

Figure 2.2 Sketch of flow domain and force balance for planar perturbation type.

Figure 2.3 Sketch of flow domain and force balance for axisymmetric perturbation type.

Figure 2.4 Sketch of orientation of normal unit vector $\mathbf{n}$ and tangential unit vector $\mathbf{t}$ for stress balance equation at interface of the fluid.

Figure 4.1 Staggered grid used to solve the rupture problem. The values of $h_{i}$ are prescribed at mesh points $r_{i}$, and values of $u_{i}$ are prescribed at $r_{i+1 / 2}=\left(r_{i}+r_{i+1}\right) / 2$.

Figure 4.2 Thickness evolution profile of a Newtonian sheet under a planar perturbation at $S / A=1 / \pi^{2}$. The initial perturbation grows over time leading to a sheet rupture at $t=1.52$.

Figure 4.3 Evolution of sheet thickness at $x=0$ for different meshes at $S / A=1 / \pi^{2}$, The results recover predictions of Ida and Miksis [27].

Figure 5.1 Planar and axisymmetric perturbations amplitude $\epsilon(t=$ $1)=\zeta(t=1)-\zeta_{0}$ as a function of $S / \hat{A}$ for Newtonian liquids. $\epsilon>0$ corresponds to perturbations that grow and lead to sheet rupture in a finite time.

Figure 5.2 Evolution of sheet thickness at $x=0$ or $r=0$ at $S / \hat{A}=1 / 10 \pi^{2}$ for both planar and axisymmetric perturbation.

Figure 5.3 Planar perturbation amplitude $\epsilon(t=1)=\zeta(1)-\zeta_{0}$ as a function of $S / \hat{A}$ an liquid properties. $\epsilon>0$ corresponds to perturbations that grow and lead to sheet rupture.

Figure 5.4 Axisymmetric perturbation amplitude $\epsilon(t=1)=\zeta(1)-$ $\zeta_{0}$ as a function of $S / \hat{A}$ an liquid properties. $\epsilon>0$ corresponds to perturbations that grow and lead to sheet rupture

Figure 5.5 Sheet rupture time as a function of liquid properties for $S / \hat{A}=1 / \pi^{2}$.

Figure 5.6 Thickness profile for $D e=1$ and different polymer-tosolvent viscosity ratio at $\mathrm{t}=1.5$.

Figure 5.7 Net force profile of Newtonian fluid under a planar perturbation in the vicinity of rupture point when thickness reaches $5 \%$ of the undisturbed film thickness. 
Figure 5.8 Net force profile of Newtonian fluid under an axisymmetric perturbation in the vicinity of rupture point when thickness reaches $15 \%$ of the undisturbed film thickness.

Figure 5.9 Net force profile Oldroyd fluid for a $D e=1$ and $\eta_{r}=10$ under a planar perturbation in the vicinity of rupture point when thickness reaches $5 \%$ of the undisturbed film thickness.

Figure 5.10 Velocity profile for Newtonian and Oldroyd-B fluids for planar perturbation type. Viscoelastic parameters were set to be $D e=1$ and $\eta_{r}=10$. And $t_{f}$ represents the moment when final thickness reaches $5 \%$ of the undisturbed film thickness.

Figure 5.11 Dynamics of film thickness at $x=0$ close to the rupture time $t_{b}$. The sheet thickness follows the form $h \approx\left(t_{b}-t\right)^{\alpha}$.

Figure 5.12 Temporal thickness evolution for Newtonian and GNM models for $\eta_{r}=2$. Profiles show that non-Newtonian effects are barely perceptible for low values of $\eta_{r}$.

Figure 5.13 Temporal thickness evolution for Newtonian and GNM models for $\eta_{r}=20$. Profiles show that non-Newtonian effects are more pronounced in the latter stage of rupture. Substantially effects on the rupture time are only observed for a high Deborah number.

Figure 5.14 Net force profiles of GNM fluid for a $D e=1$ and $\eta_{r}=10$ under a planar perturbation in the vicinity of rupture point when thickness reaches $5 \%$ of the undisturbed film thickness.

Figure 5.15 Net force profiles of Newtonian fluid under a planar perturbation in the vicinity of rupture point when thickness reaches $5 \%$ of the undisturbed film thickness.

Figure 5.16 Velocity profile for Newtonian and GNM fluids for planar perturbation type. Viscoelastic parameters were set to be $D e=$ 1 and $\eta_{r}=10$. And $t_{f}$ represents the moment when final thickness reaches $5 \%$ of the undisturbed film thickness.

Figure 5.17 Viscosity ratio profile along the axil direction for $D e=1$ and $\eta_{r}=10$ in an intermediate time $t=1.52$ and in the final time $t=2.15$.

Figure 6.1 Sketch of flow domain for a straight line retraction in a planar liquid sheet.

Figure 6.2 Sketch of flow domain for hole retraction in a planar liquid sheet.

Figure 6.3 Sketch of orientation of normal unit vector $\mathbf{n}$ and tangential unit vector $\mathbf{t}$ for stress balance equation at interface of the fluid.

Figure 6.4 Sketch of the initial thickness profile, which is assumed to be semicircular cap followed by a straight line. The curvature has a initial negative value for the circular cap and then jumps to zero for the straight line.

Figure 7.1 Initial thickness profile gets smoother as the value of $\alpha$ is augmented. 
Figure 7.2 Stagerred grid used to solve retraction problem. Values of $f_{i}$ are prescribed at mesh points $r_{i}$, and values of $u_{i}$ are prescribed at $r_{i+1 / 2}=\left(r_{i}+r_{i+1}\right) / 2$

Figure 7.3 Validation of the numerical procedure for straight line retraction in Newtonian fluid with $O h=10$ by comparing with Savva and Bush results [31]

Figure 7.4 Validation of the numerical procedure for hole retraction in Newtonian fluid with $O h=10$ by comparing with Savva and Bush results [31]

Figure 8.1 Tip velocity of straight line for Oldroyd-B model with $D e=1$ at $O h=10$ regime.

Figure 8.2 Tip velocity of a circular hole for Oldroyd-B model with $D e=1$ at $O h=10$ regime.

Figure 8.3 Thickness profile for Oldroyd-B model with $D e=1$ at $t=1$ in $O h=10$ regime.

Figure 8.4 Net force for Oldroyd-B model with $D e=1$ and $\eta_{r}=10$ at $t=1$

Figure 8.5 Tip velocity for GNM model with $D e=1$ at $O h=10$ regime.

Figure 8.6 Longitudinal velocities profile for GNM model with $D e=1$ and $\eta_{r}=10$ at $O h=10$ regime.

Figure 8.7 Viscosity profile for GNM model with $D e=1$ and $\eta_{r}=10$ at $O h=10$ regime.

Figure 8.8 Tip velocity of a hole for Oldroyd-B model with $D e=1$ at $O h=1$ regime.

Figure 8.9 Tip velocity of a hole for Oldroyd-B model with $D e=1$ at $\mathrm{Oh}=0.5$ regime.

Figure 8.10 Tip velocity of a hole for Oldroyd-B model with $D e=1$ at $O h=0.1$ regime.

Figure 8.11 Thickness profile for Oldroyd-B model with $D e=1$ at $t=3$ in $O h=1$ regime.

Figure 8.12 Thickness profile for Oldroyd-B model with $D e=1$ at $t=3$ in $O h=0.5$ regime.

Figure 8.13 Thickness profile for Oldroyd-B model with $D e=1$ at $t=1$ in $O h=0.1$ regime.

Figure 8.14 Thickness profile for Oldroyd-B model with $D e=1$ at $t=3$ in $O h=1$ regime.

Figure 8.15 Thickness profile for Oldroyd-B model with $D e=1$ at $t=3$ in $O h=0.5$ regime.

Figure 8.16 Thickness profile for Oldroyd-B model with $D e=1$ at $t=3$ in $O h=0.1$ regime. 
"Let us strive for the impossible. The great achievements throughout history have been the conquest of what seemed the impossible."

Charles Chaplin . 


\section{List of Abreviations}

\section{Greek Symbols}

$\rho$ - Density

$\sigma$ - Surface tension

$\eta$ - Viscosity for Newtonian fluid

$\dot{\gamma}$ - Rate-of-strain tensor

$\kappa$ - Interface curvature

$\tau$ - Stress tensor

$\tau^{p}$ - Polymeric stress tensor

$\tau^{s}$ - Solvent stress tensor

$\eta_{p}$ - Polymeric viscosity

$\eta_{s}$ - Solvent viscosity

$\eta_{r}$ - Polymer-to-solvent viscosity ratio

$\eta_{0}$ - Zero-extensional-rate viscosity

$\eta_{\infty}$ - Infinity-extensional-rate viscosity

$\dot{\epsilon}$ - Rate-of-extension

$\tau_{v i s}$ - Characteristic time for high $O h$

$\tau_{\text {inv }}$ - Characteristic time for low $\mathrm{Oh}$

$\Phi$ - Van der Waals potencial

$\lambda$ - Relaxation time

$\omega$ - Perturbation growth rate

$\alpha$ - Perturbation wave number

$\alpha_{c}$ - Critical perturbation wave number

\section{Roman Symbols}

$W e$ - Weber number

$U_{c}$ - Taylor-Cullick velocity

Oh - Ohnesorge number

De - Deborah number

UCM - Upper convected Maxwell

H - Undisturbed thickness

$u_{0}$ - Tip velocity

$L$ - Wavelength or sheet longitudinal extend

$S$ - Capillary to viscous force ratio

$\hat{A}$ - Van der waals to viscous force ratio

$P$ - Mechanical pressure 


\section{Introduction}

\section{1}

\section{Motivation}

Thin liquid sheets or curtains are ubiquitous in a wide range of natural and industrial applications, making their study interest to both scientist and engineers. The breakup of a liquid sheet is desirable in myriad applications, such as atomization process, ink-jet printers, microfluidic, novel devices, liquid propellant combustion in the aerospace industry as well as geophysical balances and ocean-atmosphere exchanges [1]. Conversely, the instabilities leading to the breakup process can be very damaging in other applications such as in curtain coating, where the stability of liquid curtain formed is crucial for the effectiveness of the coating process [2].

Seminal analyses on instability of thin liquid sheets were conducted by Lord Rayleigh in the nineteenth century. Based on Fourier's theorem, he determined the displacement of surfaces of thin jets moving in a still fluid with the same viscosity [8]. More than half a century later, in a sequence of three contributions, Taylor studied the formation [9], the propagation of waves [10] and the disintegration of liquid sheets [11]. Afterwards, motivated by curtain coating process, Brown [12] experimentally studied the shape and stability of a falling liquid curtain. By comparing the momentum flux pushing down a hole that may appear in the curtain with capillary force that tends to open the hole, he proposed a simple stability criterion which defines a critical Weber number, below which the curtain breaks. The Weber number is a dimensionaless parameter defined as the ratio between inertial and surface tension forces

$$
W e=\frac{\rho q U}{2 \sigma},
$$

where $\rho$ is the fluid density, $q$ is the flow rate per unit width, $U$ is curtain velocity and $\sigma$ is the surface tension. He observed that $q$ has to be high enough such that $W e>1$ to prevent curtain breakup. This simple criterion is not satisfied in some cases reported in the literature. Lin [13] showed that a falling curtain of finite length is linearly stable at all conditions. Finnicum et al.[14] 
were able to obtain stable curtain in flow conditions that violate Brown's criteria. Further experimental analysis of the flow response under a localized perturbation were presented by Roche et al. [15] and de Lucca [16]. Karim et al.[17] showed that viscous forces delay the retraction speed of the rim of a hole that is formed in the curtain, stabilizing the liquid curtain.

For complex fluids, as most coating liquids, the force balance that governs the flow and evolution of disturbances in a liquid sheet becomes more complicated. For liquids that contain dissolved long flexible polymer molecules, viscoelastic tensile stresses may appear because of the strong extensional flow that occurs near to the rupture region and affect the growth rate of any perturbation. Becerra and Carvalho [18] studied curtain breakup of dilute high molecular weight polymer solutions and showed that viscoelastic stresses delay the onset of curtain breakup. To understand the mechanism of atomization of gel propellants, Yang et al.[19] investigated the breakup characteristics of a power-law liquid sheet using a linear stability model applied to a two dimensional planar flow. They observed that as shear viscosity rises, the disturbance growth rate falls. Hence, the viscous dissipation substantially affects the flow stability. Non-Newtonian properties may play an important role in the stabilization of a thin liquid sheet, but little is known about the physical mechanisms associated with the stabilization.

The breakup process of a liquid sheet can be separated in two distinct and consecutive steps: the growth of a small perturbation until the sheet pinchesoff forming a hole and the hole expansion until the complete disintegration of the liquid sheet. The two steps are governed by different flow mechanisms with capillary forces having different roles. The flow that may lead to the sheet pinch-off is driven by van der Walls attraction and resisted by capillary and viscous forces. The growth of a hole that eventually may lead to the curtain breakup is driven by surface tension forces acting on the rim.

\section{2}

\section{Literature review}

\subsection{1}

\section{Sheet rupture}

A free liquid film is composed by a thin liquid sheet sandwiched between two fluid bulk phases. This is vulnerable to the existence of spontaneous fluctuations and waves at its surfaces. In very thin layers, van der Walls attraction becomes strong enough that even those small fluctuation can destabilize the liquid sheet, leading to its complete breakup. Rupture of soap 
films, coalescence of emulsions and fusion os lipid bilayers or of biological membranes all involve such a rupture process.

Lifshitz [20] developed a macroscopic theory of the attractive van der Waals force between bodies of large characteristic dimensions compared to interatomic distances. In his studies, he concludes that the force of mutual attraction on the surface of each body is inversely proportional to $h^{3}$, where $h$ is the film thickness. Based on the quantum field theory method, Dzyaloshinskii and Pitaevskii have found a general formula for the calculations of the van der Waals force for an arbitrary inhomogeneous medium [21] and later extended the theory of Lifshitz to study liquid films [22].

Theoretical analysis has been presented seeking the fundamental understanding of the dynamics of free thin liquid sheets rupture driven by van der Waals attraction. Vrij [23] investigated the stability of free a thin liquid sheet against small and spontaneous thickness fluctuations. Based on simple pressure balance and mass conservation, he proposed that films are unstable with respect to fluctuations with wavelength larger than a critical value given by $\alpha_{c}=\left[\left(2 \pi^{2} \sigma\right) /\left(\frac{d^{2} \Phi}{d h^{2}}\right)\right]^{1 / 2}$ where $\phi$ is the potencial associated with van der Waals force. He also calculated a lifetime and critical thickness of an unstable film.

Given that free boundaries further increase the complexity of the problem, mathematical tools have been incorporated in the studies. Taking the advantage that thin liquid sheets have the distinct characteristic of being slender, the long wavelength theory can be used to simplify equations. A remarkable and seminal work using this tool was developed by Prevost and Gallez [24]; they took into account the long-wave nature of the response of liquid film under a periodic disturbance and derived a partial differential equation to describe the interface shape evolution subjected to viscous force, surface tension and van der Waals force. Based on this simplification, they performed a nonlinear stability analysis to explore the effect of hydrodynamics nonlinearities on the rupture process of a thin free film. Results showed that nonlinearities accelerate the rupture process.

Following this work, Erneux and Davis [25] used the full Navier-Stokes equations for free films with an extra term to incorporate the van der Waals attraction to derive a two coupled nonlinear evolution equations for longitudinal component of velocity $(u)$ and thickness of the film $(h)$

$$
\begin{aligned}
& \frac{\partial h}{\partial t}+\frac{\partial}{\partial x}(u h)=0 \\
& \frac{\partial u}{\partial t}+u \frac{\partial u}{\partial x}+\frac{\partial \Phi}{\partial x}-3 S \frac{\partial^{3} h}{\partial x^{3}}-\frac{4}{h} \frac{\partial}{\partial x}\left(h \frac{\partial u}{\partial x}\right)=0
\end{aligned}
$$


where $\Phi=A(2 \pi h)^{-3}$ is the van der Waals potencial, $A$ is the non-dimensional van der Waals coefficient and $S$ is non-dimensional surface tension.

Using the set of Eqs. 1-1 and 1-2, they first performed the linear stability analysis, which consists of finding a characteristic equation for the growth rate, $\omega$, of an imposed perturbation. For a Newtonian fluid under a planar perturbation, it is given by

$$
\omega^{2}+4 \alpha^{2} \omega+\frac{3}{2} \alpha^{2}\left(S \alpha^{2}-2 \hat{A}\right)=0
$$

The critical parameters that mark the transition from stable to unstable films are those that make $\omega=0$ in Eq. 1-3. For a critical wave number ${ }^{1} \alpha_{c}=\pi$, the linear stability criterion proposed by Erneux Davis [25] is given by the ratio between surface tension and van der Waals forces

$$
\frac{S}{\hat{A}}=\frac{2}{\pi^{2}}
$$

Based on this results, they used $S$ as the bifurcation point and investigated the nonlinear problem in the vicinity of this point and confirmed that nonlinear terms contribute to the acceleration of the rupture phenomenon, as suggested by Prevost and Gallez [24].

Effects of surface tension gradient on the rupture dynamics were investigated by Wit and Gallez [54]. Extending the analysis presented by Erneux and Davis [25], they studied the planar rupture of a free film with insoluble surfactants. From a linear stability analysis, they concluded that Marangoni effect does not affect the stability criterion, however increasing the surfactant concentration, the growth rate of the instabilities decreases and thus increases the rupture time of the film. Results were also observed in the numerical solution of the full set of equations.

Looking to delineate the influence of different nonlinearities, Sharma et al. [55] investigated the dynamics of free film rupture in a simplified Erneux and Davis's [25] equations, where inertia and unsteady effects were neglected. They concluded that the influence of inertia and nonlinear viscous correction are found not to be very significant for small amplitudes, and so the simplified model is useful since it reduces the computational time by orders of magnitude. However, for relatively large amplitude disturbances and close to the critical wave number, they observed that destabilization due to inertia and nonlinearity of van der Waals forces plays an important role.

A more general and realistic approach was used by Shugai et al. [56];

${ }^{1}$ Critical wave number is the wave number that admits $\omega=0$. 
waves were treated as packets localized in space rather than monochromatic waves prescribed by temporal stability approach. Based on Erneux and Davis's equations [25], they rewrote evolution equations in an equivalent form by introducing disjoining pressure instead of van der Waals potential. Performing a spacial-temporal stability analysis, they observed that, even in linear approximation, the long-range intermolecular forces strongly affect the evolution of initially localized disturbances; a negative disjoining pressure caused by van der Waals forces strongly promotes instability. Consequently, the absolute growth rate and speed of spreading of disturbances increase as the thickness of undisturbed film decreases.

Presence of chemical reactions in a liquid film gives rise to variations in density and viscosity since these properties depend on the components concentration and temperature of the liquid. Matar and Spelt [57] explored the dynamics of thin free films with reaction-driven density and viscosity variations and observed the existence of a number of competing effects; the heat released during the reactions processes decreases the density and viscosity of the liquid and gives rise to thermocapillary effects. Viscosity reduction and thermocapillary effects are destabilizing, promoting film thinning and van der Waals-driven rupture. However, the decrease in density leads to film expansion to satisfy mass conservation, which is stabilizing mechanism.

Effects of non-newtonian properties on the stability of thin free liquid sheets were recently investigated by Yang et al. [19]. Performing a linear stability analysis and numerical investigation in a power-law fluid, they observed that increasing non-Newtonian properties caused two different impacts in sheet instability; velocity at the interface increases with non-Newtonian effects, and larger velocities augment aerodynamics instabilities, in the other hand, disturbances can be damped out due by the viscous dissipation effects. The resulting effect of non-Newtonian properties on the liquid sheet stability depends on the combination of two aspects.

Self-similarity analysis is another important mathematical tool to study long wavelength models for van der Waals-driven rupture of free thin liquid sheets. Using this tool, Vaynblat et al. [43] investigated the influence of disturbances type in the rupture sheet dynamics. They performed an analysis of planar and axisymmetric perturbation evolution near a rupture singularity and were able to obtain the corresponding similarity solutions. From this analysis, they observed that van der Waals forces, viscosity, and inertia are asymptotically significant, but the action of surface tension is negligible near to rupture point.

Based on the set of equations proposed by Erneux and Davis [25], Ida 
and Miksis [27] studied the dominant force balances in the evolution equation using similarity-type solution in the temporal and spatial vicinity of rupture. Assuming that close to breaking point van der Waals and viscous forces are dominant, with inertia and surface tension being negligible, they found an analytical result for values of the exponents for the similarity solutions. They were able to confirm this assumption by solving numerically the set of partial differential equations; by examining the relative size of each term of equation near to breaking, they observed that van der Waals and viscous forces dominate the evolution.

Self-similarity analyses for non-Newtonian fluids were addressed by Thete et al. [44]. They investigated the evolution of a power-law liquid film toward the space-time singularity and were able to determine scaling exponent governing the time evolution of the film thickness based on non-Newtonian properties of the fluid. From the analysis, they were able to construct similarity profiles for the interface shape close to rupture point.

Complementing Vaynblat et al. [43] work, Thete et al. [58] recently studied the dynamics of film rupture in two limit case; inertialess and inviscid flow regime. The analysis revealed that the scaling exponents for the selfsimilarity solution of the film thickness in any of three regimes, i.e Stokes, inertial or inertial-viscous, has the same value regardless of the disturbance type - planar or axisymmetric. They also observed that inviscid limit agrees with Thete et al. [44] results for a power-law fluid under non-Newtonian conditions where viscous forces become negligible.

\section{2 .2}

\section{Sheet retraction}

After the pinch-off, a small hole (axisymmetric perturbation) or straight line (planar perturbation) appears in the liquid curtain, which may grow with time and lead it to full disintegration of the sheet as shown in the snapshot sequence presented by Karim et al. [17] during an experimental investigation of the effect of viscosity on liquid curtain stability. 

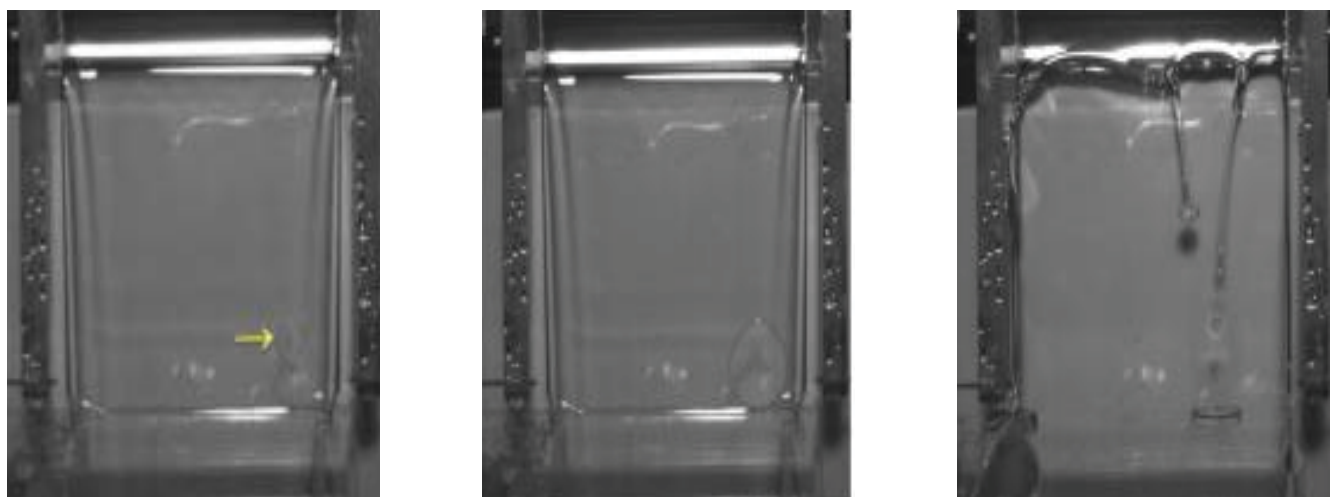

Figure 1.1: Desintegration process in a Newtonian liquid sheet caused by the growth of a small role due to action of surface tension [17].

Pioneer studies of film retraction were developed by Dupré [60] in the nineteenth century. Based on the assumption that surface energy is purely converted into kinetic energy, he deduced the retraction speed of the film. Later, using global mass and momentum balance, Taylor [9] and Cullick [62] independently studied the motion of the free edge of a circular hole in a liquid sheet. They calculate the film retraction speed, $U_{c}$, which was a factor of $\sqrt{2}$ smaller than the velocity predicted by Dupré [60]

$$
U_{c}=\sqrt{\frac{2 \sigma}{\rho H}}
$$

Experimental results obtained by McEntee and Mysels [63] for soap film with a thickness greater than $0.1 \mu \mathrm{m}$ confirmed Cullick-Taylor theory.

Seeking to understand the effect of viscosity on film retraction, Debrégeas et al. [65] experimentally investigated the bursting of a very viscous free liquid film, with the absence of any surfactant. Experiments were performed by forming circular suspended films of polymer polydimethylsiloxane (PDMS). They observed that the retraction speed was not constant, rather the hole radius grows exponentially

$$
r_{0}(t)=R_{0} \exp \left(\frac{t}{\tau}\right)
$$

here $R_{0}$ is initial size of the role and $\tau=1.4 \mu H / 2 \sigma$ is defined as the exponential rise time. Debrégeas et al. [65] also observed that the fluid is no longer collected by the rim, which is a new behavior in the thin liquid films retraction. They attributed the absence of the rim and the exponential behavior of retraction velocity to the viscoelastic behavior of the fluid.

Following this work, Brenner and Gueyffier [66] have numerically investigated the planar retraction of thin free planar films. The mathematical formu- 
lation was based on Erneux and Davis's equation [25] modified to include the complete formula for the mean curvature. Three distinct regimes were studied: low viscosity, intermediate viscosity (viscosity equals to twenty times of the water) and high viscosity (viscosity equal to one million times of the water). They observed that for the low viscosity case, the retraction speed was constant and agreed with Taylor-Culick law, also there was the formation of a cylindrical rim and capillary wave disturbances are generated ahead of the rim. For the case of intermediate viscosity, they observed that, after a transient period, the retraction velocity reached Taylor-Culick law, and there was a non-cylindrical rim formation with the absence of capillary waves. In this case, they noted that the rim presented a horizontal length prportional to the characteristic dimension $\mu / \rho U 0$. In the case of very high viscosity, as previously observed by Debrégeas et al [65], the rim completely disappeared. However, they were not able to find the exponential retraction velocity as proposed by Debrégeas et al. [65].

Song and Tryggvason [67] numerically studied the influence of surrounding fluid in the film retraction dynamics. They observed that for a finite-size domain, there is a reduction in the edge velocity as the rim grows, however, for a larger domain, the retraction velocity was not affected by the surrounding fluid.

Complementing Brenner and Gueyffier [66] and Song and Tryggvason [67] studies, Sunderhauf et al. [41] numerically investigated the acceleration of the rim during the planar retraction process for inertia- and viscositydominated flow regimes. Looking to capture more detailed flow information in the transient region, they performed the numerical studies for full NavierStokes equations. The inverse of the Ohnesorge $(O h)$ number was used to characterize the flow

$$
\Gamma_{v}=\frac{1}{O h}=\sqrt{\frac{2 \rho H \sigma}{\mu^{2}}} .
$$

They observed that in high viscosity regimes, or for a small $\Gamma_{v}$, the motion of the rim affects a large mass of liquid, which slows down the rim acceleration. They also observed that considering a long time limit, all cases reached the Taylor-Cullick velocity.

Through lubrication theory, Savva and Bush [31] examined the influence of viscosity and geometry in the transient phase of film retraction. Using the Ohnesorge number (Oh) as the main flow parameter, they were able to identify the three regimes presented Brenner and Gueyffier [66] in the planar geometry. Based on this results, they proposed an analytical prediction for the temporal 
edge speed $\left(u_{0}\right)$ and displacement $\left(x_{0}\right)$ in high $O h$ condition for planar sheet retraction

$$
\begin{aligned}
& u_{0}(t)=U_{c} \sqrt{\frac{t}{\pi \tau_{v i s}}}, \\
& x_{0}(t)=\frac{2}{3 \sqrt{\pi}}\left(\frac{t}{\tau_{\text {vis }}}\right)^{3 / 2} H,
\end{aligned}
$$

here $\tau_{v i s}=O h H / U_{c}$ is the characteristic retraction time, for high $O h$ regime. They also observed that the maximum film thickness had linear temporal growth regardless of $O h$ number.

For a circular geometry, Savva and Bush [31] observed that the retraction dynamics are markedly different, which confirms the relevance of geometry in sheet retraction. In high $O h$ limit, they found an analytical expression for the temporal displacement of the hole $\left(r_{0}\right)$ in the early stages of retraction:

$$
r_{0}(t)=R_{0} \exp \left(\frac{t}{2 \tau_{v i s}}\right)
$$

The expression proposes an exponential behavior for the hole growth, as predicted by Debregeas et al.'s studies. In this sense, this behavior is not associated with viscoelasticity as reported Debregeas et al., but a generic feature of the hole expansion in a viscous liquid sheet. The difference between Eqs. 1-9 and 1-10 lies on the contribution of surface tension coming from the extra curvature in the azimuthal direction; the extra curvature opposes to expansion during the early stage of retraction.

Gordillo et al. [70] studied numerically and analytically the capillary retraction phenomenon of a viscous fluid in a film surrounded by a viscous and an inviscid gas. They performed the numerical simulation for full Navier-Stokes equations and for an approximation using long wavelength theory. Results showed a good agreement between both methods. Based on the asymptotic method, they developed an analytical solution for the interface shape for viscous and inviscid surrounding.

Recently, through finite element numerical simulation, Vilonne et al. [68] investigated the retraction of a circular viscoelastic liquid film for confined and unconfined geometry. Three constitutive models were evaluated: Oldroyd-B, Giesekus, and Phan-Thien-Tanner. Results for the Oldroyd-B model showed that, when inertia is relevant, the absence of wall effects caused moderate viscoelasticity influence and the film retraction dynamics tends to TaylorCullick law. However, in the presence of wall-effects oscillations appeared in the flow which enhanced viscoelasticity effects. For viscous dominant flows, 
the unconfined geometry tends to dynamics predicted by Debregeas et al. with some deviation due to viscoelasticity effects. In the case of confined geometry, viscous dominant flow presented the absence of oscillations, with the hole speed going through a maximum and then monotonically decaying to zero. Models that took into account non-constant viscosity, such as Geisekus and PhanThien-Tanner, showed no significant difference in the dynamics of the film retraction compared to Oldroyd-B model.

\section{3}

\section{Dissertation goals}

Most of the available studies on the stability of thin liquid sheets consider the liquid as Newtonian. In the case of non-Newtonian fluids, analyses were limited to power-law fluids, especially in the rupture process.

In this sense the goal of this work is to provide a complete study of the breakup process of viscoelastic thin liquid sheets. The investigation is performed for both stages; perturbations growth in the liquid sheet and subsequent sheet retraction process. For the first stage, the investigation of perturbation growth, the goals of this work are:

- Develop a linear stability analysis and propose a stability criterion for axisymmetric perturbations in a Newtonian fluid film based on Erneux and Davis's work [25].

- Solve the full set of the evolution equation numerically, find the stability criteria for planar and axisymmetric perturbation and compare the prediction with the analytical results of linear stability analysis. Also analyze theoretically and numerically the effects of non-Newtonian properties on the stability criteria, for both types of perturbations.

- Investigate the nonlinear influence of non-Newtonian properties in the perturbation growth rate and rupture time.

- Study the effect of non-Newtonian properties on the self-similarity solution.

For the sheet retraction, the objectives of this work are:

- Develop a mathematical formulation based on long-wavelength approximation taking into account the non-Newtonian properties of the fluids.

- Implement a numerical methodology based on Finite Difference Method to solve the set of equations.

- Investigate the influence of non-Newtonian properties in the retraction speed and in the thickness profile. 


\section{4 \\ Scope of work}

This work is organized in Part I and Part II. Part I is devoted to present the dynamics of rupture of non-Newtonian thin free liquid sheets. This part is divided into chapters as follow: Chapter $\S 2$ presents the complete mathematical formulation of the problem. Chapter $\S 3$ describes the stability analysis for the Newtonian axisymmetric and non-Newtonian planar problem. Chapter $\S 4$ describes the aspects of the numerical methodology employed. And Chapter $\S 5$ presents and discusses extensively the results obtained in this part of the research.

Part II is devoted to present the dynamics of the retraction of a nonNewtonian liquid sheet. It is split into following chapters: Chapter $\S 6$ presents the mathematical formulation used to describe the problem. Chapter $\$ 7$ brings numerical strategy necessary to solve the retraction problem, given that the velocity boundary condition at the tip is part of the solution. And Chapter $\S 7$ presents and discusses the results obtained in this part.

Finally, Chapter $\$ 9$ presents the general conclusion and suggestions for future works. 


\section{Part I}

\section{Rupture process}




\section{2}

\section{Mathematical modeling}

Flows of non-Newtonian fluids, such as viscoelastic fluids, have a very particular dynamics. The complexity associated with the stress field and the vast number of different mechanical responses to imposed rate-of-strains do not allow a single and simple mathematical formulation to describe the flow behavior of non-Newtonian fluids. In this sense, the choice of a suitable model becomes essential for the correct flow description and model. This chapter describes the mathematical formulation used to describe the rupture of a thin liquid sheet, which includes the mass and momentum conservation equations, stress tensor model with the associated boundary and initial conditions.

\section{1}

\section{Conservations equations}

A liquid sheet, bounded by a passive gas, is considered thin enough that van der Waals forces are significant, while thick enough that continuum hypothesis is valid. The flow, considered two-dimensional and isothermal, is described by the mass and momentum conservation equations for incompressible liquids

$$
\nabla \cdot \mathbf{u}=0
$$

and

$$
\rho \frac{D \mathbf{u}}{D t}=\nabla \cdot \mathbf{T}+\nabla \Phi
$$

$\rho$ is the liquid density, and $\mathbf{u}$ is the velocity field. The van der Waals potential $\Phi$ is defined as

$$
\Phi=\frac{A}{2 \pi h^{3}},
$$

$A$ is the Hamaker constant and $h$ is the film thickness.

The stress tensor $\mathbf{T}=-p \mathbf{I}+\boldsymbol{\tau}$ is split into an isotropic ${ }^{1}$ and a deviatoric component. The isotropic component $-p \mathbf{I}$ is the mechanical pressure and

\footnotetext{
${ }^{1}$ Isotropic component is invariant under rotation of the axes of reference [26].
} 
defined as $p=-1 / 3 \operatorname{tr}(\mathbf{T})$. The non-isotropic or deviatoric stress tensor has the distinctive property of being due entirely to the existence of the motion of the fluid [26]. For Newtonian fluids the deviatoric component is linearly proportional to the rate-of-strain tensor $\dot{\gamma}$

$$
\tau=\mu \dot{\gamma}
$$

where $\mu$ is a parameter of proportionality between rate-of-strain and stress tensor called viscosity, which depends significantly on the temperature; for isothermal flow it becomes a constant [26].

The Newtonian constitutive equation is the simplest equation for a viscous fluid and accurately describes the rheological behavior of low molecular weight liquids and even high polymers at a very slow rate of deformation [48]. However, for liquids of complex molecular structure, especially for those consisting in long molecular chains, some emulsion and mixtures, the Newtonian expression (2-3) is not accurate to represent the deviatoric stress at only moderate rate of deformation. Stress anisotropy, flow deformation history, and strain rate-dependent viscosity are flow characteristics that invalidate the linear Newtonian behavior.

A large number of constitutive models have been proposed in order to incorporate non-Newtonian rheological behavior of fluids in the stress tensor. Developing a constitutive model is a combination of art and science to look for an appropriate tensorial expression for stress as a function of deformation to match observed behavior, where there is no recipe to follow, however, there are some constraints to ensure the physical sense of the equations. The constraints are [49];

- Stress is independent of the coordinate system used to describe it, thus, the stress is a second-order tensor where the constitutive equation must be frame invariant.

- The stress tensor is symmetric for most materials and for all conventional polymer melts and solutions;

- The response of a material to imposed stress or to imposed deformation is the same for all observers, thus there is a requirement of material objectivity.

Section 2.1.1 presents one model where using a minor modification of the Newtonian fluid constitutive equation, one can include the idea of rateof-deformation dependent viscosity in the stress tensor. Although this model cannot describe memory flow effect and time-dependent elastic effects, there 
are many industrial flow problems in which the non-Newtonian viscosity effects are of paramount importance, and hence the model is useful [39].

Section 2.1.2 presents a simple, although still complex, model to describe the rheological behavior of a viscoelastic fluid. As viscoelastic liquids have a more convoluted molecular structure, a more complex stress tensor models is required in order to include memory flow effect and stress anisotropy.

\subsection{1}

\section{Generalized Newtonian model}

For many engineering problems, the most important property of macromolecular fluid is the dependence of viscosity to the deformation rate. Given that viscosity can change up to two orders of magnitude with the rate of strain, it cannot be ignored in flow calculations, lubrications problems, extruder operations and polymer processing calculations. Therefore, one earliest empiricism to be introduced was a modification of Newton's law of viscosity, where viscosity is allowed to vary with the rate-of-strain, called Generalized Newtonian model, GNM. GNM is phenomenological model that assumes that applied flow only changes the dissipation rate in the fluid [69] i.e, its viscosity, and does not change the tensorial structure of the Newtonian constitutive model, so the constitutive law can be written as:

$$
T=-p \mathbf{I}+\eta \dot{\gamma}
$$

Here $\eta$ is the viscosity written as a function of magnitude of rate-of-strain tensor $\dot{\gamma}$, i.e $\eta=\eta(\dot{\gamma})$, were $\dot{\gamma}$ is second tensorial invariant of rate of strain tensor $I I$.

$$
\dot{\gamma}=\sqrt{\frac{1}{2} I I}
$$

Typically, polymer solutions have a viscosity that falls with increasing shear rate, which is called shear-thinning behavior. For flows where there is no shear stress, the rate-of-strain $\dot{\gamma}$ reduces to a rate-of-extension. Usually, the extensional viscosity of polymer solutions increases with the rate-of-extension, which is called an extensional-thickening behavior. The empirical viscosity model presented in Eq. 2-6 describes that behavior.

$$
\eta(\dot{\epsilon})=\eta_{\infty}+\left(\eta_{0}-\eta_{\infty}\right)\left(1+(\lambda \dot{\epsilon})^{2}\right)^{\frac{n-1}{a}}
$$

Thus viscosity model interpolates between the zero-extensional-rate vis- 
cosity $\eta_{0}$ to the infinity-extensional-rate viscosity $\eta_{\infty}$. A relaxation time $\lambda$ sets the crossover extensional rate, $n$ is the power-law index and the constant $a$ sets the size and the curvature of the crossover region from the Newtonian-plateau with viscosity $\eta_{0}$ to a high-viscosity-plateau $\eta_{\infty}$, as sketched in Fig. 2.1.

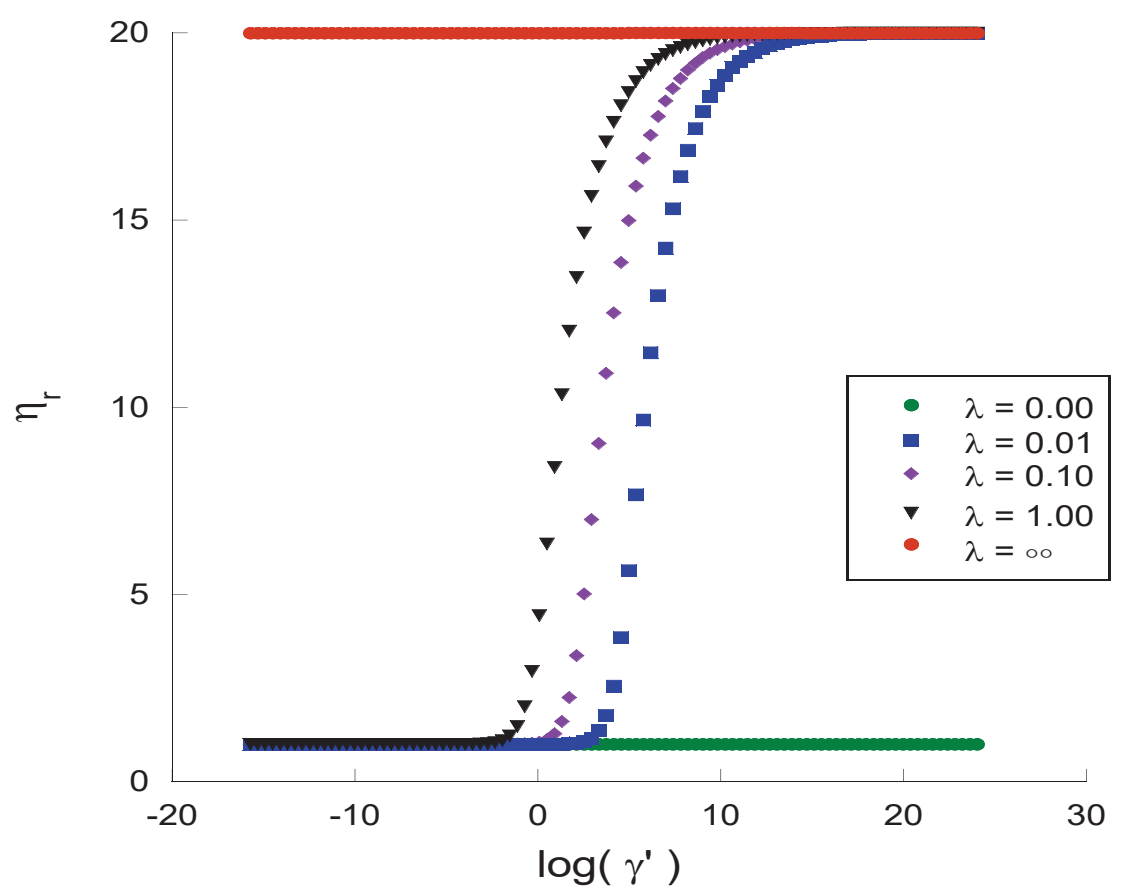

Figure 2.1: Sketch of the rheological behavior of Generalized Newtonian model under an extensional flow.

Generalized Newtonian model is a simple extension of the linear relation between the stress and the velocity gradient in the classical Newtonian constitutive law.

\subsection{2}

\section{Oldroyd-B model}

For a viscoelastic material, constitutive equations are mathematical relationships that allow one to calculate the stress in a liquid, given the flow history. They are often derived from constitutive models, i.e a set of assumptions and idealizations about the molecular or structural forces and motions that produces stress. By comparing a constitutive equation derived from a given model with measured stresses, it is possible to test the model and further use it to predict a flow or test ideas of polymer molecular physics [46].

An idealization of a viscoelastic fluid was proposed by Maxwell over a century ago [39]. In his theory, Newton's law of viscosity and Hooke's law of elasticity are combined to arrive at a simple equation that describes a 
viscoelastic liquid, which in simple shear flow is given as

$$
\tau_{x y}+\frac{\mu}{G} \frac{\partial \tau_{x y}}{\partial t}=\mu \dot{\gamma}_{x y}
$$

where $\dot{\gamma}_{x y}$ is $x y$-component of the rate-of-strain tensor, $\mu$ is the fluid viscosity and $G$ is the elastic modulus. For steady-state flow this equation simplifies into the Newtonian fluid with viscosity $\mu$, i.e $\tau_{x y}=-\mu \dot{\gamma}_{x y}$. For sudden changes in stress, time derivative term dominates, and one has an expression that, integrated with respect to time, gives the Hookean solid with elastic modulus $G$, i.e $\tau_{x y}=-G \gamma_{x y}$.

Equation 2-7 can be generalized and written in the tensor form, where $\mu$ is replaced by $\eta_{0}$, which is the zero-shear-rate viscosity and $\mu / G$ is by a time constant $\lambda$, often called relaxation time

$$
\boldsymbol{\tau}+\lambda \frac{\partial}{\partial t} \boldsymbol{\tau}=\eta_{0} \dot{\gamma}
$$

However, Hooke's law is valid only for infinitesimal displacement gradients, in this sense it is reasonable to expect that Maxwell's equation is subjected to the same restriction. For large strain rates, Eq. 2-8 fails in taking into account the principle of frame invariance (one of the constrains presented in the begin of this section), which is one of chief among of principles of continuum mechanics. The frame invariance requires that the relationship between the stress tensor and deformation gradient tensor be independent of any rotation of the body or the observer [46]. In this sense, Oldroyd proposed that the frame independence could be recovered by writing the Maxwell's equation 2-8 in a frame of reference that is convected or deformed with the material element. To do so, one must reinterpret the time derivative $\partial / \partial t$ as a time derivative in a convected coordinate system. There are two simple convected coordinate systems; one where the base coordinate vectors are parallel to material lines, or contravariant base vectors, an other the set of base vectors are normal to material planes, or covariant base vectors. The contravariant base vector, also named upper-convected derivative is, in general, preferable since it has molecular basis and a better agreement with experimental data [46]. In this sense, time derivative of Eq. 2-8 is rewritten as

$$
\frac{\partial \boldsymbol{\tau}}{\partial t}=\stackrel{\nabla}{\boldsymbol{\tau}}=\frac{D}{D t} \boldsymbol{\tau}-(\boldsymbol{\tau} \cdot \nabla \boldsymbol{u})^{T}-(\boldsymbol{\tau} \cdot \nabla \boldsymbol{u})
$$

Writing the deviatoric stress tensor $\boldsymbol{\tau}$ as a superposition of solvent and polymer contributions, $\tau=\tau_{s}+\tau_{p}$, gives rise to the simplest, yet complex, model that introduces memory and stress anisotropy and also maintains 
the independence of the framework: the Oldroyd-B model. In this model, the solvent constitutive equation follows Newton's law, $\tau_{s}=\eta_{s} \dot{\gamma}$, and the polymeric term is given by Eq. 2-8 written in a convected coordinate system described in Eq. 2-9 named the upper-convected Maxwell model (UCM) [46]

$$
\tau^{p}+\lambda \stackrel{\nabla}{\tau^{p}}=\eta_{p} \dot{\gamma}
$$

where $\eta_{p}$ is the polymeric viscosity.

\section{2}

\section{Boundary conditions}

Equations (2-1, 2-2 and 2-10) are solved using Cartesian coordinate system for planar perturbation, and cylindrical coordinate system for axisymmetric perturbation, as sketched in Figs 2.2 and 2.3. The local sheet thickness is defined by the function $h=h(x, t)$ for the planar case and $h=h(r, t)$ for the axisymmetric problem.

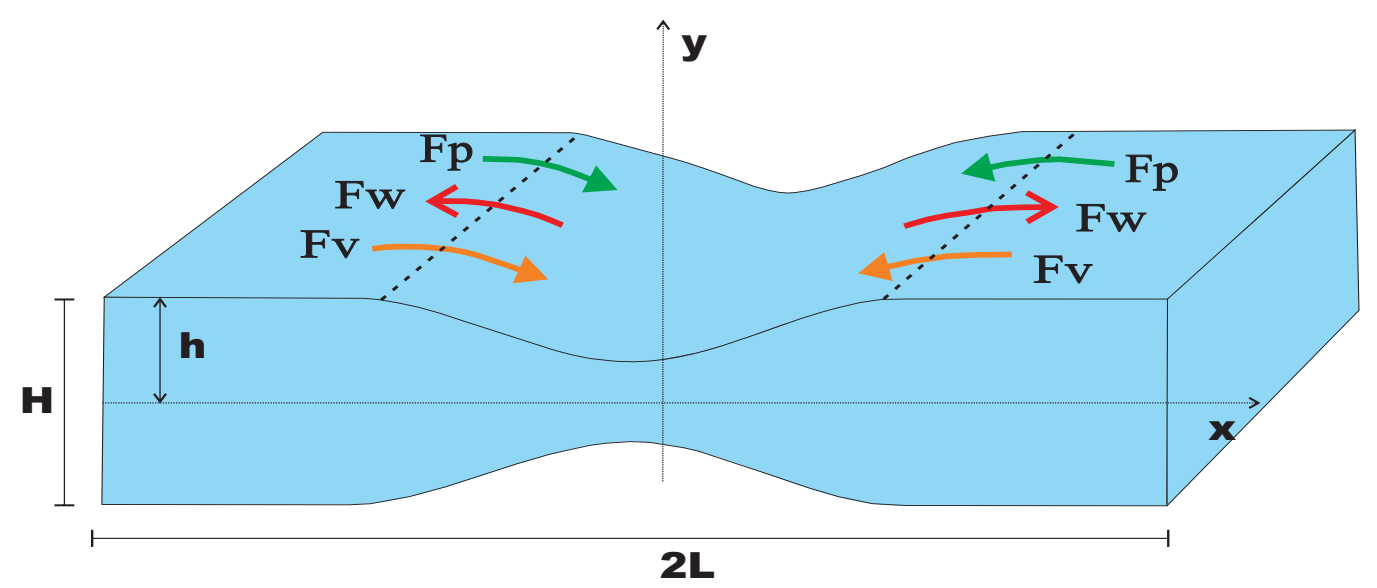

Figure 2.2: Sketch of flow domain and force balance for planar perturbation type. 


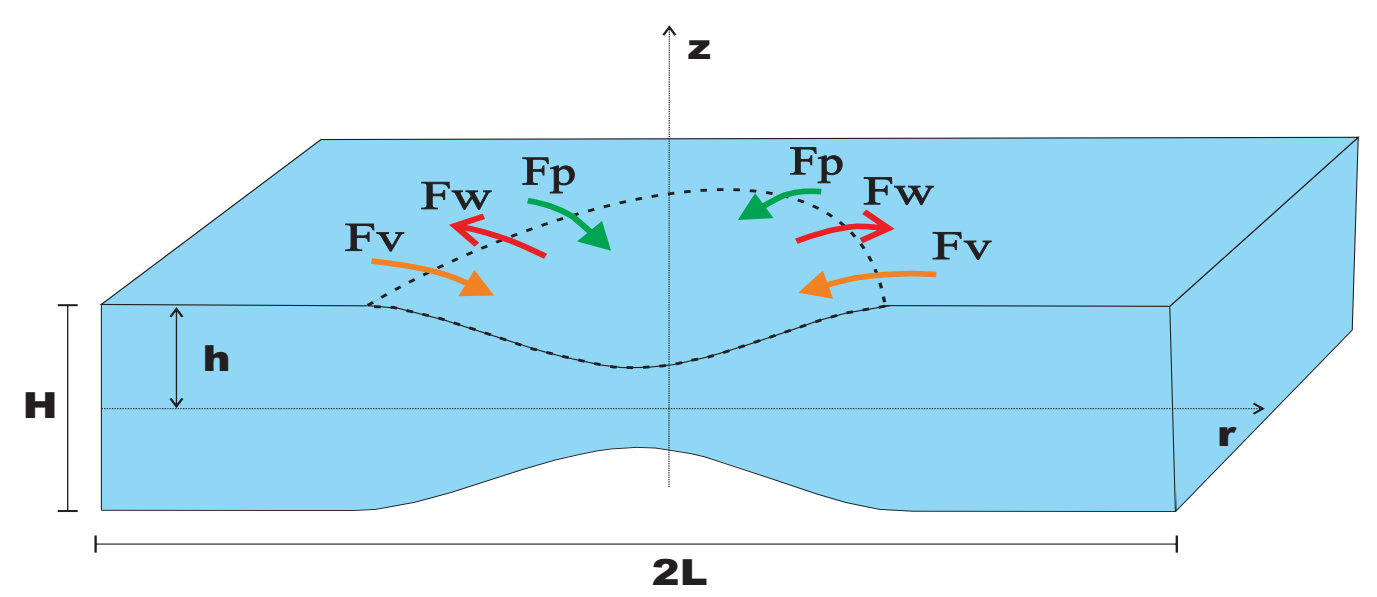

Figure 2.3: Sketch of flow domain and force balance for axisymmetric perturbation type.

Boundary conditions along the liquid-gas interface are defined at $z=h$ and correspond to force balance along the normal and tangential directions, and kinematic condition, as sketched in Fig 2.4.

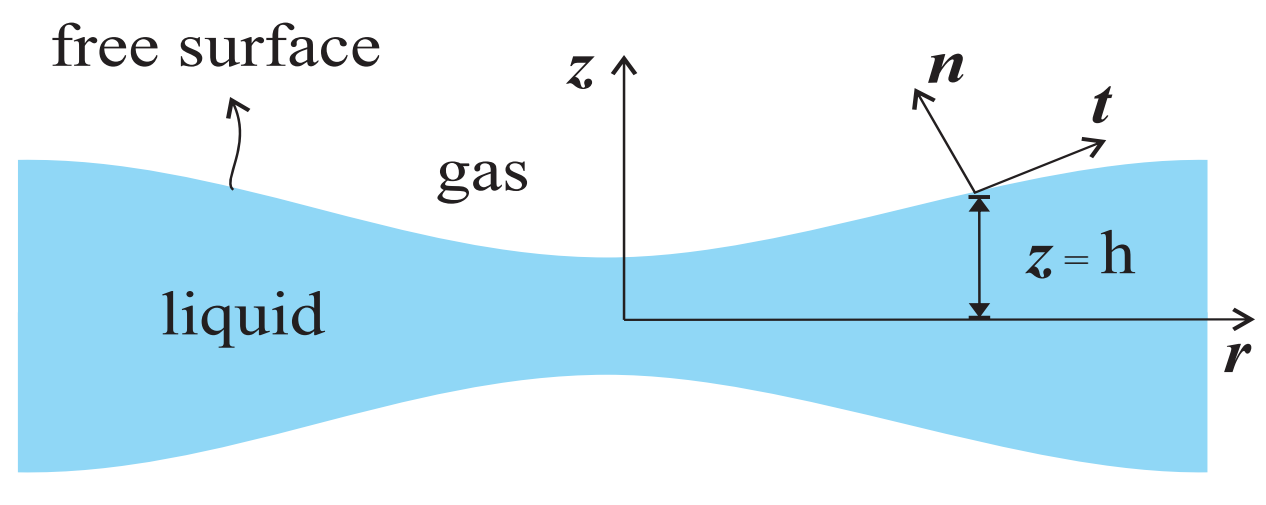

Figure 2.4: Sketch of orientation of normal unit vector $\mathbf{n}$ and tangential unit vector $\mathbf{t}$ for stress balance equation at interface of the fluid.

For force balance, considering that the gas viscosity is much smaller than liquid viscosity, thus $\mu_{\text {gas }} / \mu_{\text {liquid }} \approx 0$, and free of inertial effects, the shear stress at gas-liquid interface is zero and hence the normal liquid stress must be balanced with capillarity stress.

$$
\begin{aligned}
& \mathbf{n} \cdot(\mathbf{n} \cdot \mathbf{T})=\sigma \kappa, \\
& \mathbf{t} \cdot(\mathbf{n} \cdot \mathbf{T})=0 .
\end{aligned}
$$


Here $\sigma$ is the surface tension, which is assumed to be constant, $\kappa$ is the curvature of the free surface. Kinematic condition imposes that there is no flow through the liquid-gas interface in such way that the surface must be moving with the liquid velocity along the free surface:

$$
\mathbf{n} \cdot \mathbf{u}=\frac{\partial h}{\partial t}
$$

$\mathbf{n}$ and $\mathbf{t}$ are the normal and tangential unit vectors, which for the axisymmetric problem, are written as:

$$
\begin{aligned}
& \mathbf{t}=\left[\left(1+\left(\frac{\partial h}{\partial r}\right)^{2}\right)^{-1 / 2} \mathbf{e}_{r}, \quad \frac{\partial h}{\partial r}\left(1+\left(\frac{\partial h}{\partial r}\right)^{2}\right)^{-1 / 2} \mathbf{e}_{z}\right] \\
& \mathbf{n}=\left[-\frac{\partial h}{\partial r}\left(1+\left(\frac{\partial h}{\partial r}\right)^{2}\right)^{-1 / 2} \mathbf{e}_{r}, \quad\left(1+\left(\frac{\partial h}{\partial r}\right)^{2}\right)^{-1 / 2} \mathbf{e}_{z}\right]
\end{aligned}
$$

The initial condition assumes a stationary sheet of constant thickness $H$, which is perturbed by a cosine function with amplitude $H^{\prime}$ and wavelength $L$, centered at $x=0$ or $r=0$ :

$$
h(x, t=0)=H-H^{\prime} \cos \left(\frac{\pi x}{L}\right)
$$

\section{3}

\section{Lubrication approximation}

The presence of free boundaries increases the complexity of the mathematical problem. However, the slenderness of the fluid film leads to a large ratio of characteristics lengths along the directions parallel and perpendicular to the flow. In this sense, the system of equations can be simplified using long wavelength asymptotic expansions $[42,31]$.

Section 2.3.1 describes the mathematical procedures for planar perturbation model, based on the two-dimensional Cartesian conservation equations and 2.3.2 for axisymmetric perturbation model, based on the two-dimensional cylindrical conservations equations. 


\subsection{1}

\section{Planar model}

For a two dimensional planar flow, Eqs.(2-1) and (2-2) in Cartesian coordinates are written as:

$$
\begin{aligned}
& \frac{\partial U}{\partial x}+\frac{\partial V}{\partial y}=0 \\
& \rho\left(\frac{\partial U}{\partial t}+U \frac{\partial U}{\partial x}+V \frac{\partial U}{\partial y}\right)=-\frac{\partial P}{\partial x}-\frac{\partial \Phi}{\partial x}+\frac{\partial T_{x x}}{\partial x}+\frac{\partial T_{x y}}{\partial y} \\
& \rho\left(\frac{\partial V}{\partial t}+U \frac{\partial V}{\partial x}+V \frac{\partial V}{\partial y}\right)=-\frac{\partial P}{\partial y}-\frac{\partial \Phi}{\partial y}+\frac{\partial T_{x y}}{\partial x}+\frac{\partial T_{y y}}{\partial y}
\end{aligned}
$$

For an planar slender sheet, symmetric about $y=0$, all the fields can be expanded about $y=0[31]$ :

$$
\begin{aligned}
& U(x, y, t)=u(x, t)+u_{2}(x, t) y^{2}+\ldots \\
& V(x, y, t)=v_{1}(x, t) y+v_{3}(x, t) y^{3}+\ldots \\
& P(x, y, t)=p(x, t)+p_{2}(x, t) y^{2}+\ldots \\
& \Phi(x, y, t)=\phi(x, t)+\phi_{2}(x, t) y^{2}+\ldots \\
& T_{x x}(x, y, t)=\tau_{x x}(x, t)+\tau_{x x, 2}(x, t) y^{2}+\ldots \\
& T_{x y}(x, y, t)=\tau_{x y}(x, t) y+\tau_{x y, 2}(x, t) y^{3}+\ldots \\
& T_{y y}(x, y, t)=\tau_{y y}(x, t)+\tau_{y y, 2}(x, t) y^{2}+\ldots
\end{aligned}
$$

Replacing the above expansions in Eqs.(2-16), (2-17) and (2-38), matching the powers of $y$ and taking only the leading order terms, the equations are reduced to:

$$
\begin{aligned}
& v_{1}=-\frac{\partial u}{\partial x} \\
& \rho\left(\frac{\partial u}{\partial t}+u \frac{\partial u}{\partial x}\right)=-\frac{\partial p}{\partial x}-\frac{\partial \phi}{\partial x}+\frac{\partial \tau_{x x}}{\partial x}+\tau_{y x} .
\end{aligned}
$$

Combining Taylor expansions with Eqs.(2-11), (2-12) and (2-13), using unit normal $\mathbf{n}$ and tangential $\mathbf{t}$ defined in 2-14, the boundary conditions along 
the free surface are written as:

$$
\begin{aligned}
& {\left[\left(\frac{\partial h}{\partial x}\right)^{2}\left(\tau_{x x}-p\right)+\frac{\partial h}{\partial r} \tau_{y x} h+\tau_{y y}-p\right]\left[1+\left(\frac{\partial h}{\partial r}\right)^{2}\right]^{-1}=\sigma \kappa} \\
& {\left[-\frac{\partial h}{\partial x} \tau_{x x}+\left[1-\left(\frac{\partial h}{\partial x}\right)^{2}\right] \tau_{y x} h+\frac{\partial h}{\partial x} \tau_{y y}\right]\left[1+\left(\frac{\partial h}{\partial x}\right)^{2}\right]^{-1}=0} \\
& \frac{\partial h}{\partial t}+u \frac{\partial h}{\partial x}-v_{1} h=0
\end{aligned}
$$

Given a slender liquid sheet, the long wavelength approximation leads to $\left(\frac{\partial h}{\partial r}\right)^{2} \approx 0$, consequentially $\left[1+\left(\frac{\partial h}{\partial r}\right)^{2}\right] \approx 1$, hence Eqs. 2-22 and 2-23 can be simplified to

$$
\begin{aligned}
& -p=\sigma \kappa-\frac{\partial h}{\partial r} \tau_{y x} h-\tau_{y y} \\
& \tau_{y x} h=\frac{\partial h}{\partial x}\left(\tau_{x x}-\tau_{y y}\right) .
\end{aligned}
$$

Pressure gradient can be found by combining the derivative of Eqs. 2-25 and 2-26

$$
-\frac{\partial p}{\partial x}=\sigma \frac{\partial \kappa}{\partial x}-\frac{\partial \tau_{y y}}{\partial x}
$$

Replacing Eqs. 2-26 and 2-27 into 2-21 one can find the lubrication equation for radial velocity

$$
\frac{\partial u}{\partial t}+u \frac{\partial u}{\partial x}-\frac{\sigma}{\rho} \frac{\partial \kappa}{\partial x}+\frac{1}{\rho} \frac{\partial \phi}{\partial x}-\frac{1}{\rho} \frac{\partial \tau_{x x}}{\partial x}+\frac{1}{\rho} \frac{\partial \tau_{y y}}{\partial x}-\frac{1}{\rho h} \frac{\partial h}{\partial x}\left(\tau_{x x}-\tau_{y y}\right)=0
$$

In the long wave expansion, curvature $\kappa$ is approximated as:

$$
\kappa=\frac{\partial^{2} h}{\partial x^{2}}\left(1+\left(\frac{\partial h}{\partial x}\right)^{2}\right)^{-3 / 2} \approx \frac{\partial^{2} h}{\partial x^{2}}
$$

Combining Eqs.(2-20) and (2-24) one can find the thickness evolution equation

$$
\frac{\partial h}{\partial t}+u \frac{\partial h}{\partial x}+h \frac{\partial u}{\partial x}=0
$$

Equations 2-28 and 2-30 form the general lubrication system that can be applied to any type of fluid. Using Oldroyd-B model presented in subsection 2.1.2, one can split the stress tensor into the solvent component $\tau^{s}$ and a polymeric stress $\tau^{p}$. 
The set of equations may be written in terms of the following dimensionless variables:

$$
\begin{gathered}
x^{*}=\frac{x}{L}, \quad h^{*}=\frac{h}{H}, \quad u^{*}=\frac{u}{\frac{\eta_{s}}{\rho L}}, \quad t^{*}=\frac{t}{\frac{\rho L^{2}}{\eta_{s}}} \\
\tau_{x x}^{p *}=\frac{\tau_{x x}^{p}}{\frac{\eta_{s}^{2}}{\rho L}}, \quad \tau_{y y}^{p *}=\frac{\tau_{y y}^{p}}{\frac{\eta_{s}^{2}}{\rho L}} .
\end{gathered}
$$

Dropping the ${ }^{*}$ to simplify the notation, the evolution equations for dimensionless longitudinal velocity $u$, film thickness $h$ and polymeric stress tensor components are:

$$
\begin{aligned}
\frac{\partial h}{\partial t}+u \frac{\partial h}{\partial x}+h \frac{\partial u}{\partial x}=0 & \begin{array}{r}
\frac{\partial u}{\partial t}+u \frac{\partial u}{\partial x}-3 S \frac{\partial^{3} h}{\partial x^{3}}-\frac{3 \hat{A}}{8 h^{4}} \frac{\partial h}{\partial x}-\frac{4}{h} \frac{\partial h}{\partial x} \frac{\partial u}{\partial x}-4 \frac{\partial^{2} u}{\partial x^{2}}-\frac{\partial \tau_{x x}^{p}}{\partial x} \\
+\frac{\partial \tau_{y y}^{p}}{\partial x}-\frac{\tau_{x x}^{p}}{h} \frac{\partial h}{\partial x}+\frac{\tau_{y y}^{p}}{h} \frac{\partial h}{\partial x}=0 \\
\tau_{x x}^{p}+D e\left(\frac{\partial \tau_{x x}^{p}}{\partial t}+u \frac{\partial \tau_{x x}^{p}}{\partial x}-2 \tau_{x x}^{p} \frac{\partial u}{\partial x}\right)
\end{array}=-2 \eta_{r} \frac{\partial u}{\partial x} \\
\tau_{y y}^{p}+D e\left(\frac{\partial \tau_{y y}^{p}}{\partial t}+u \frac{\partial \tau_{y y}^{p}}{\partial x}+2 \tau_{y y}^{p} \frac{\partial u}{\partial x}\right) & =2 \eta_{r} \frac{\partial u}{\partial x}
\end{aligned}
$$

The problem is governed by the following dimensionless parameters: (a) $S=\frac{\sigma H \rho}{6 \eta_{s}^{2}}$ that represents the ratio of capillary and inertial to viscous forces; (b) $\hat{A}=\frac{A \rho l^{2}}{6 \pi H^{3} \eta_{s}^{2}}$ that represents the ratio of van der Walls to viscous forces; (c) Deborah number $D e=\frac{\lambda \eta_{s}}{L^{2} \rho}$, which measures the ratio of the liquid relaxation time to a characteristic time of the flow, and (d) $\eta_{r}=\frac{\eta_{p}}{\eta_{s}}$ that is the ratio of the polymeric and solvent viscosity. Newtonian flow is recovery by setting $D e=0$ and $\eta_{r}=0$. 


\subsection{2}

\section{Axisymmetric model}

For a two dimensional axisymmetric flow, Eqs.(2-1) and (2-2) in cylindrical coordinates are written as:

$$
\begin{aligned}
& \frac{\partial}{\partial r}(U r)+\frac{\partial}{\partial z}(V r)=0 \\
& \rho\left(\frac{\partial U}{\partial t}+U \frac{\partial U}{\partial r}+V \frac{\partial U}{\partial z}\right)=-\frac{\partial P}{\partial r}-\frac{\partial \Phi}{\partial r}+\frac{1}{r} \frac{\partial}{\partial r}\left(r T_{r r}\right)+\frac{\partial}{\partial z} T_{z r}-\frac{T_{\theta \theta}}{r} \\
& \rho\left(\frac{\partial V}{\partial t}+U \frac{\partial V}{\partial r}+V \frac{\partial V}{\partial z}\right)=-\frac{\partial P}{\partial z}-\frac{\partial \Phi}{\partial z}+\frac{1}{r} \frac{\partial}{\partial r}\left(r T_{z r}\right)+\frac{\partial}{\partial z} T_{z z}
\end{aligned}
$$

Following the approached detailed in subsection 2.3.1, now for an axisymmetric slender sheet, symmetric about $z=0$, where expansion for $T_{r r}$ is equal to expansion for $T_{x x}$ and $T_{r z}$ is equal to $T_{x y}$, it is possible to replace the expansions in Eqs.(2-36), (2-37) and (2-38), match powers of $z$ and take the lowest order terms, to reduce equations as following:

$$
\begin{aligned}
& v_{1}=-\frac{\partial u}{\partial r}-\frac{u}{r} \\
& \rho\left(\frac{\partial u}{\partial t}+u \frac{\partial u}{\partial r}\right)=-\frac{\partial p}{\partial r}-\frac{\partial \phi}{\partial r}+\frac{1}{r} \frac{\partial}{\partial r}\left(r \tau_{r r}\right)+\tau_{z r}-\frac{\tau_{\theta \theta}}{r} .
\end{aligned}
$$

Similarly to subsection 2.3.1, boundary conditions along the free surface are written as:

$$
\begin{aligned}
& {\left[\left(\frac{\partial h}{\partial r}\right)^{2}\left(\tau_{r r}-p\right)+\frac{\partial h}{\partial r} \tau_{z r} h+\tau_{z z}-p\right]\left[1+\left(\frac{\partial h}{\partial r}\right)^{2}\right]^{-1}=\sigma \kappa} \\
& {\left[\frac{\partial h}{\partial r} \tau_{r r}+\left[1-\left(\frac{\partial h}{\partial r}\right)^{2}\right] \tau_{r z} h-\frac{\partial h}{\partial r} \tau_{z z}\right]\left[1+\left(\frac{\partial h}{\partial r}\right)^{2}\right]^{-1}=0} \\
& \frac{\partial h}{\partial t}+u \frac{\partial h}{\partial r}-v_{1} h=0
\end{aligned}
$$

In the long wavelength limit, $\left(\frac{\partial h}{\partial r}\right)^{2} \approx 0$, therefore Eqs.(2-41) and (2-42) can be written as:

$$
\begin{aligned}
-p & =\sigma \kappa-\tau_{z z} \\
\tau_{r z} & =\frac{1}{h} \frac{\partial h}{\partial r}\left(\tau_{r r}-\tau_{z z}\right) .
\end{aligned}
$$

Taking the derivative of Eq. 2-44 and plugging it and Eq. 2-45 into Eq. 
2-40, the radial velocity is described by the following transport equation

$$
\begin{aligned}
\frac{\partial u}{\partial t}+u \frac{\partial u}{\partial r}-\frac{\sigma}{\rho} \frac{\partial \kappa}{\partial r}+\frac{1}{\rho} \frac{\partial \phi}{\partial r}-\frac{1}{r \rho} \frac{\partial}{\partial r}\left(r \tau_{r r}\right)+\frac{1}{\rho} \frac{\partial \tau_{z z}}{\partial r} \\
-\frac{\tau_{r r}}{h \rho} \frac{\partial h}{\partial r}+\frac{\tau_{z z}}{h \rho} \frac{\partial h}{\partial r}+\frac{\tau_{\theta \theta}}{r}=0
\end{aligned}
$$

where the curvature $\kappa$ is approximated to

$$
\begin{aligned}
\kappa & =\frac{\partial^{2} h}{\partial r^{2}}\left(1+\left(\frac{\partial h}{\partial r}\right)^{2}\right)^{-3 / 2}-\frac{\partial h}{\partial r}\left[r\left(1+\left(\frac{\partial h}{\partial r}\right)^{2}\right)^{1 / 2}\right]^{-1 / 2}, \\
\kappa & \approx \frac{\partial^{2} h}{\partial r^{2}}-\frac{1}{r} \frac{\partial h}{\partial r}
\end{aligned}
$$

Equation(2-39) combined with Eq. (2-43) give the thickness time evolution of the liquid sheet

$$
\frac{\partial h}{\partial t}+h \frac{\partial u}{\partial r}+u \frac{\partial h}{\partial r}+\frac{h u}{r}=0
$$

Equations 2-46 and 2-48 form the general set of lubrication equations for an axisymmetric flow. The Oldroyd-B model is used to describe the stress field.

The set of equations can be written in terms of the following dimensionless variables:

$$
\begin{aligned}
& r^{*}=\frac{r}{L}, \quad h^{*}=\frac{h}{H}, \quad u^{*}=\frac{u}{\frac{\eta_{s}}{\rho L}}, \quad t^{*}=\frac{t}{\frac{\rho L^{2}}{\eta_{s}}}, \\
& \tau_{r r}^{p *}=\frac{\tau_{r r}^{p}}{\frac{\eta_{s}^{2}}{\rho L}}, \quad \tau_{z z}^{p *}=\frac{\tau_{z z}^{p}}{\frac{\eta_{s}^{2}}{\rho L}}, \quad \tau_{\theta \theta}^{p *}=\frac{\tau_{\theta \theta}^{p}}{\frac{\eta_{s}^{2}}{\rho L}} .
\end{aligned}
$$

Dropping the ${ }^{*}$ to simplify the notation, the evolution equations for dimensionless longitudinal velocity $u$, film thickness $h$ and polymeric stress 
tensor components are:

$$
\begin{aligned}
& \frac{\partial h}{\partial t}+\frac{1}{r} \frac{\partial}{\partial r}(h r u)= 0 \\
& \frac{\partial u}{\partial t}+u \frac{\partial u}{\partial r}-3 S\left(\frac{\partial^{3} h}{\partial r^{3}}+\frac{1}{r} \frac{\partial^{2} h}{\partial r^{2}}-\frac{1}{r^{2}} \frac{\partial h}{\partial r}\right)-\frac{4}{h}\left[\frac{\partial}{\partial r}\left(\frac{h}{r} \frac{\partial}{\partial r}(u r)\right)-\frac{u}{2 r} \frac{\partial h}{\partial r}\right] \\
& \quad-\frac{3 \hat{A}}{8 h^{4}} \frac{\partial h}{\partial r}+\frac{1}{r} \frac{\partial}{\partial r}\left(h \tau_{z z}^{p}\right)-\frac{1}{h r} \frac{\partial}{\partial r}\left(h r \tau_{r r}^{p}\right)+\frac{\tau_{\theta \theta}^{p}}{r}=0 \\
& \tau_{r r}^{p}+D e\left(\frac{\partial \tau_{r r}^{p}}{\partial t}+u \frac{\partial \tau_{r r}^{p}}{\partial r}-2 \tau_{r r}^{p} \frac{\partial u}{\partial r}\right)=2 \eta_{r} \frac{\partial u}{\partial r} \\
& \tau_{z z}^{p}+D e\left(\frac{\partial \tau_{z z}^{p}}{\partial t}+u \frac{\partial \tau_{z z}^{p}}{\partial r}+\frac{\tau_{z z}^{p}}{r} \frac{\partial}{\partial r}(r u)\right)=-\frac{2 \eta_{r}}{r} \frac{\partial}{\partial r}(r u) \\
& \tau_{\theta \theta}^{p}+D e\left(\frac{\partial \tau_{\theta \theta}^{p}}{\partial t}+u \frac{\partial \tau_{\theta \theta}^{p}}{\partial r}-2 \tau_{\theta \theta}^{p} \frac{u}{r}\right)=2 \eta_{r} \frac{u}{r}
\end{aligned}
$$

Dimensionless parameters that govern the axisymmetric flow, $S, \hat{A}, D e$ and $\eta_{r}$, are the same ones that govern the planar flow and defined in section 2.3.1. 


\section{Linear stability}

Linear stability analysis is usually the first step taken to investigate flow stability. This analysis helps to understand the dynamics of the linearized flow. It results in the determination of a critical parameter that determines whether the perturbation grows with time or ultimately decays to zero.

Erneux and Davis [25] have performed the stability analysis for Newtonian sheet subjected to a small perturbations, and they have shown that a uniform thickness sheet of a Newtonian liquid is linearly stable to planar perturbation when the capillary forces are strong enough when compared to the van der Waals forces. Following the same procedure, section 3.1 presents an evaluation of the linear stability analysis for a viscoelastic fluid under a planar perturbations, and section section 3.2 for Newtonian fluid under an axisymmetric perturbations.

\section{1}

\section{Planar model}

The first approach to perform linear stability analysis is to consider the steady-state solutions of the problem; radial velocity $\bar{u}$, thickness profile $\bar{h}$ and stress components $\bar{\tau}_{x x}, \bar{\tau}_{y y}$. Steady-state solutions only depend on axial position and satisfy the governing equations. Afterwards, a time- and spatial-dependent perturbation of some type is added in such way that solutions are represented as

$$
\begin{aligned}
& h(x, t)=\bar{h}(x)+h^{\prime}(x, t), \\
& u(x, t)=\bar{u}(x)+u^{\prime}(x, t), \\
& \tau_{x x}(x, t)=\bar{\tau}_{x x}(x)+\tau_{x x}^{\prime}(x, t), \\
& \tau_{y y}(x, t)=\bar{\tau}_{y y}(x)+\tau_{y y}^{\prime}(x, t) .
\end{aligned}
$$

For this problem, stability analysis is performed for an static film 
submitted to an infinitesimal disturbance. The steady-state solution is:

$$
\begin{aligned}
& \bar{u}=0, \\
& \bar{h}=\frac{1}{2}, \\
& \bar{\tau}_{x x}=\bar{\tau}_{y y}=0 .
\end{aligned}
$$

The perturbed time-dependent solutions are written as:

$$
\begin{aligned}
& h(x, t)=\frac{1}{2}+h^{\prime}(x, t) \\
& u(x, t)=u^{\prime}(x, t) \\
& \tau_{x x}(x, t)=\tau_{x x}^{\prime}(x, t) \\
& \tau_{y y}(x, t)=\tau_{y y}^{\prime}(x, t)
\end{aligned}
$$

In linear theory, perturbations $h^{\prime}, u^{\prime}, \tau_{x x}^{\prime}$ and $\tau_{y y}^{\prime}$ are taken to have much smaller amplitudes than mean quantities ${ }^{1}$, i.e $|\bar{h}|>>\left|h^{\prime}\right|^{2}$. Substituting the perturbed solutions into the set of Eqs. 2-32 to 2-35, one have a system that describe the evolution of the perturbations. Doing that, products of perturbated terms emerge in the equations (for instance $u^{\prime} \partial h^{\prime} / \partial r$ ), however, as the amplitude of perturbations are considered small ${ }^{3}$ those terms are discarded, and the set of equations are written as

$$
\begin{aligned}
& \frac{\partial h^{\prime}}{\partial t}+\bar{h} \frac{\partial u^{\prime}}{\partial x}=0 \\
& \frac{\partial u^{\prime}}{\partial t}-3 S \frac{\partial^{3} h^{\prime}}{\partial x^{3}}-\frac{3}{8} \frac{A}{\bar{h}} \frac{\partial h^{\prime}}{\partial x}-4 \frac{\partial^{2} u^{\prime}}{\partial x^{2}}-\frac{\tau_{x x}^{\prime p}}{\partial x}+\frac{\partial \tau_{y y}^{\prime p}}{\partial x}=0 \\
& \tau_{x x}^{\prime p}+D e \frac{\partial \tau_{x x}^{\prime p}}{\partial t}=-2 \eta_{r} \frac{\partial u^{\prime}}{\partial x} \\
& \tau_{y y}^{\prime p}+D e \frac{\partial \tau_{y y}^{\prime p}}{\partial t}=2 \eta_{r} \frac{\partial u^{\prime}}{\partial x}
\end{aligned}
$$

Eqs. 3-1 to 3-4 form the linearized system for perturbed quantities which is the base of linear stability. Since it came from the hypothesis of small perturbations, the solutions of the linear set of equations is only valid in the beginning of growth of any disturbances, a large perturbation amplitudes

${ }^{1}$ When mean quantities are not zero due to the problem dynamics.

${ }^{2}$ Another way to represent the small amplitude of perturbations that can be found in the literature is including a parameter $\epsilon<<1$ in front of the perturbed term of the equation, i.e $h(x, t)=\bar{h}(x)+\epsilon h^{\prime}(x, t)$. However, as we work only to first order term of $\epsilon$, for simplification, we choose to neglect the parameter $\epsilon$.

${ }^{3}$ Thinking in terms of $\epsilon$, product of perturbations are of order of $\epsilon^{2}$, in this sense those terms are approximated zero and so can be negligible. 
invalidates the theory.

Since the set of equations is linear, the disturbances can be decomposed into normal modes. For static flow, the normal-mode disturbances are given by a stationary wave. Taking the dominant mode, the set of equations allow solutions in the form:

$$
\left(h^{\prime}, u^{\prime}, \tau_{x x}^{\prime}, \tau y y^{\prime}\right)=\left(h_{0}, u_{0}, \tau_{x x 0}, \tau_{y y 0}\right) e^{\omega t+i \alpha x}
$$

where $h_{0}, u_{0}, \tau_{x x 0}$ and $\tau_{y y 0}$ are eigenfunctions from the solution of this eigenvalue problem, $\alpha$ is the wavenumber in $x$-direction and $\omega$ is represented here as temporal growth/decay rate. For the stability question, the most important quantity is $R e(\omega)$, which is the real part of the exponential function. Small disturbances grow if $\operatorname{Re}(\omega)>0$ and decay if $\operatorname{Re}(\omega)<0 ; \omega=0$ impose a neutrally stable condition, marking the boundary between stable and unstable condition.

Replacing 3-5 into the set of linear Eqs. 3-1 to 3-4 one can find the algebraic characteristic equations for the growth rate of perturbations

$$
\omega^{2}+\left(4 \alpha^{2}+\frac{4 \eta_{r}}{1+D e \omega} \alpha^{2}\right) \omega+\frac{3}{2} \alpha^{2}\left(S \alpha^{2}-2 \hat{A}\right)=0
$$

which admits a root $\omega=0$ if the last coefficient is equal to zero. In this condition there is a "cutoff" wave number at which the perturbation is neutrally stable:

$$
\alpha_{c}=\sqrt{\frac{2 \hat{A}}{S}} .
$$

For a bounded domain $-1 \leq x \leq 1$, with periodic boundary conditions, the wavenumber $\alpha$ only takes distinct values given by $\alpha=n \pi$ where $(n=$ $1,2, .$.$) . Assuming the parameter \mathrm{S}$ as the bifurcation parameter ${ }^{3}$ [25], one can find the stability criterion, that for $\alpha_{c}=\pi$, is given by $S=2 \hat{A} \pi^{-2}$, which is exactly the same condition derived by Erneux and Davis [25] for a Newtonian liquid. Here it is important to note that the non-Newtonian parameters, Deborah number $D e$ and polymer-to-solvent viscosity ratio $\eta_{r}$, only appear in the term that multiplies $\omega$ and therefore have no effect on the critical stability condition.

\footnotetext{
${ }^{3} \mathrm{~A}$ bifurcation occurs when a change in a parameter causes a change in the stability of system in equilibrium. In continuous systems, this corresponds to the real part of an eigenvalue of the system passing through zero [47]; the parameter responsible for causes the change is call bifurcation parameter.
} 


\section{2}

\section{Axisymmetric model}

In order to investigate the affect of perturbation type on linear stability criterion, the same procedure presented in section 3.1 is used in this section to performance a linear stability analysis for axisymmetric perturbations. Using the same thickness and radial velocity for the steady-state solutions of section 3.1, the linearized evolution equations for Newtonian liquids are:

$$
\begin{aligned}
& \frac{\partial h^{\prime}}{\partial t}+\bar{h} \frac{\partial u^{\prime}}{\partial r}=0 \\
& \frac{\partial u^{\prime}}{\partial t}-3 S\left(\frac{\partial^{3} h^{\prime}}{\partial r^{3}}+\frac{1}{r} \frac{\partial^{2} h^{\prime}}{\partial r^{2}}-\frac{1}{r^{2}} \frac{\partial h^{\prime}}{\partial r}\right)-\frac{3 A}{8 h^{4}} \frac{\partial h^{\prime}}{\partial r}-4 \frac{\partial^{2} h^{\prime}}{\partial r^{2}}-\frac{4}{r} \frac{\partial u^{\prime}}{\partial r}=(33-9)
\end{aligned}
$$

Similarly to section 3.1, Eqs. (3-8) and (3-9) accept solutions of the form:

$$
\left(h^{\prime}, u^{\prime}\right)=\left(h_{0}, u_{0}\right) e^{\omega t+i \alpha r},
$$

which can be substituted in the evolution equations and, taking the real part, an algebraic equation for the growth rate $\omega$ for a Newtonian fluid under an axisymmetric perturbation is obtained:

$$
\omega^{2}+4 \omega \alpha^{2}+\frac{3}{2} \alpha^{2}\left(S \alpha^{2}+\frac{4 S}{L^{2}}-2 \hat{A}\right)=0
$$

The root of Eq. 3-11, $\omega=0$, only exist if the last coefficient is equal to zero, so the "cutoff" wave number at which the perturbation is neutrally stable is:

$$
\alpha_{c}=\sqrt{\frac{2 \hat{A}}{S}-\frac{4}{L^{2}}} .
$$

A critical ratio of capillary to van der Waals force $S / \hat{A}$ can be derived as a function of the perturbation wave number $\alpha_{c}$, and for $\alpha_{c}=\pi$ is given by:

$$
\frac{S}{\hat{A}}=\frac{2}{\pi^{2}+\frac{4}{L^{2}}} .
$$

The additional term that appears in the denominator in the axisymmetric problem is associated to the azimuthal curvature of the free surface. The results suggests that a smaller surface tension is needed to stabilize an axisymmetric perturbation. 


\section{4}

\section{Solution method}

This chapter presents the numerical methodology used to solve the full set of coupled non-linear equations that describes the rupture process. A secondorder finite difference method was used to discretize the spacial derivatives and an implicit Crank-Nicholson method to discretize the time derivative terms. The result is a sparse set of coupled, nonlinear algebraic system equations, which was solved using Newton's method, at each time step.

\section{1}

\section{Finite difference method}

The Finite Difference Method (thereafter FDM) is the oldest method for numerical solution of partial difference equations (PDE's), believed to have been introduced by Euler [28] in the eighteenth century. The method consist on covering the solution domain with a grid and, at each grid point, approximate the derivatives using truncated Taylor series expansion.

A staggered mesh over the interval $0<x<1$ or $0<r<1$ was used to avoid spurious oscillations in the curvature term in the long-time limit, as suggested by Savva and Bush [31]; the values of $h_{i}, \tau_{r r, i}, \tau_{z z, i}$ and $\tau_{\theta \theta, i}$ are prescribed at $r_{i}$ and the values of $u_{i}$ are prescribed at $r_{i+1 / 2}=\left(r_{i}+r_{i+1}\right) / 2$, as sketched in Fig 4.1. 


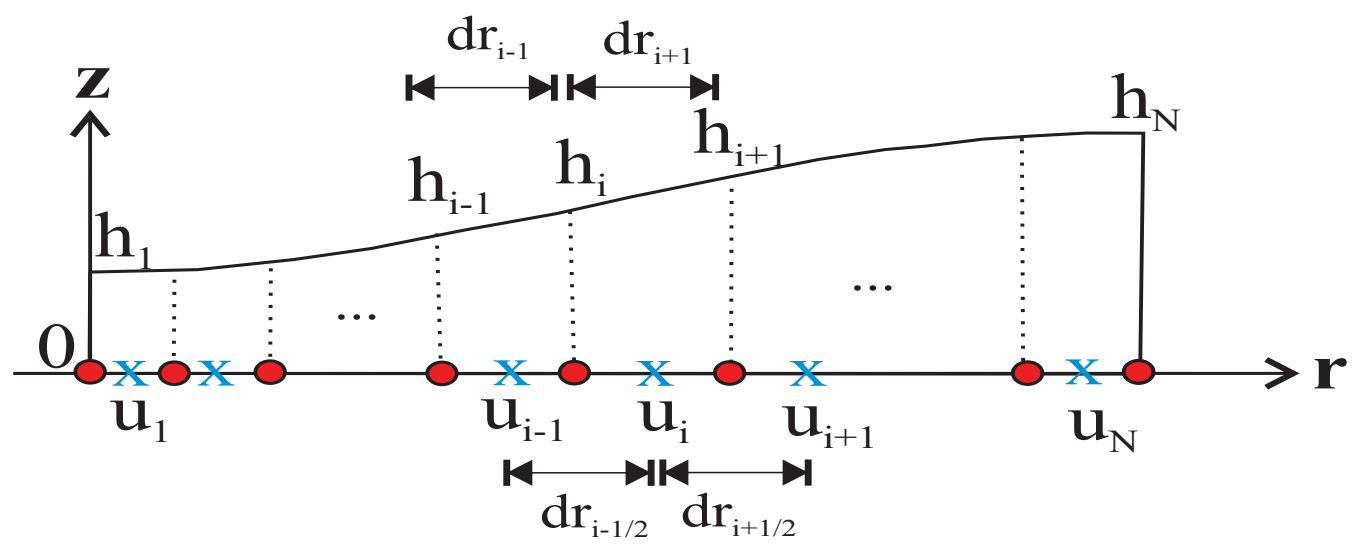

Figure 4.1: Staggered grid used to solve the rupture problem. The values of $h_{i}$ are prescribed at mesh points $r_{i}$, and values of $u_{i}$ are prescribed at $r_{i+1 / 2}=\left(r_{i}+r_{i+1}\right) / 2$.

Taylor series expansion is used to obtain approximations to first and second derivative of the variable in respect to the spatial coordinate. To performance the expansions, we consider $u(x, t), h(r, t), \tau_{r r}(r, t)$ and $\tau_{z z}(r, t)$ as continuous differentiable function, so they can be, in the vicinity of $r_{i}$, expressed as [28]:

$$
\begin{aligned}
& u(r)=u\left(r_{i+1 / 2}\right)+\left(r-r_{i+1 / 2}\right)\left(\frac{\partial u}{\partial r}\right)_{i+1 / 2}+\frac{\left(r-r_{i+1 / 2}\right)^{2}}{2}\left(\frac{\partial^{2} u}{\partial r^{2}}\right)_{i+1 / 2}+\mathcal{O} \\
& h(x)=h\left(x_{i}\right)+\left(r-r_{i}\right)\left(\frac{\partial h}{\partial r}\right)_{i}+\frac{\left(r-r_{i}\right)^{2}}{2}\left(\frac{\partial^{2} h}{\partial r^{2}}\right)_{i}+\mathcal{O} \\
& \tau_{r r}(x)=\tau_{r r}\left(x_{i}\right)+\left(r-r_{i}\right)\left(\frac{\partial \tau_{r r}}{\partial r}\right)_{i}+\frac{\left(r-r_{i}\right)^{2}}{2}\left(\frac{\partial^{2} \tau_{r r}}{\partial r^{2}}\right)_{i}+\mathcal{O} \\
& \tau_{z z}(x)=\tau_{z z}\left(x_{i}\right)+\left(r-r_{i}\right)\left(\frac{\partial \tau_{z z}}{\partial r}\right)_{i}+\frac{\left(r-r_{i}\right)^{2}}{2}\left(\frac{\partial^{2} \tau_{z z}}{\partial r^{2}}\right)_{i}+\mathcal{O} \\
& \tau_{\theta \theta}(x)=\tau_{\theta \theta}\left(x_{i}\right)+\left(r-r_{i}\right)\left(\frac{\partial \tau_{\theta \theta}}{\partial r}\right)_{i}+\frac{\left(r-r_{i}\right)^{2}}{2}\left(\frac{\partial^{2} \tau_{\theta \theta}}{\partial r^{2}}\right)_{i}+\mathcal{O}
\end{aligned}
$$

where $\mathcal{O}$ represents higher order terms. Replacing $r$ by $r_{i+1}$ and $r_{i-1}$, for $h$, $\tau_{r r}, \tau_{z z}$ and $\tau_{\theta \theta}$, and $r_{i+3 / 2}$ and $r_{i-1 / 2}$, for $u$, one can obtain expressions for the derivative values at these points. The same can be extended to any other point, such as $r_{i-2}$ and $r_{i+2}$. Expansion for $u_{i+1}$ and $u_{i-1}$ can be combined to create a central-difference scheme (CDS). Given a staggered grid, first and 
second derivatives for $u$ and $h$ are written as:

$$
\begin{aligned}
& \left(\frac{\partial u}{\partial r}\right)_{i+1 / 2}=\frac{u_{i+1}-u_{i-1}}{d r_{i+1 / 2}+d r_{i+1 / 2}} \\
& \left(\frac{\partial^{2} u}{\partial r^{2}}\right)_{i+1 / 2}=\frac{d r_{i+1 / 2} u_{i+1}+\left(d r_{i+1 / 2}+d r_{i+3 / 2}\right) u_{i}-d r_{i+3 / 2} u_{i-1}}{d r_{i+1 / 2}^{2} d r_{i+3 / 2}+d r_{i+1 / 2} d r_{i+3 / 2}^{2}} \\
& \left(\frac{\partial h}{\partial r}\right)_{i}=\frac{h_{i+1}-h_{i-1}}{d r_{i-1}+d r_{i}} \\
& \left(\frac{\partial^{2} h}{\partial r^{2}}\right)_{i}=\frac{d r_{i} h_{i+1}+\left(d r_{i}+d r_{i+1}\right) h_{i}-d r_{i+1} h_{i-1}}{d r_{i}^{2} d r_{i+1}+d r_{i} d r_{i+1}^{2}}
\end{aligned}
$$

Terms that were deleted from the right side of the expansions are called truncation errors; they measure the accuracy of the approximations and at which rate the error decreases as the spacing between two points is reduced [28]. However, truncation error depends not only on grid spacing but also on the derivative of the variables. Non-uniform grid uses a smaller $d r$ in regions where the derivative of the functions are large and larger $d r$ in regions where the function is smooth to obtain a more accurate approximation. In the present problem, the mesh was concentrated in the region near to the rupture point $r=0$, by using the function

$$
r_{i}=1-\left[\frac{N-i}{N-1}\right]^{a}
$$

where $a$ is the parameter that controls the nodal points distribution; $a=1$ recovers an uniform mesh and $a<1$ leads to a concentrated mesh in the vicinity of $r=0$.

For a matter of definitions, Eqs. 2-50 to 2-54 are written as

$$
\begin{aligned}
& \frac{\partial h}{\partial t}=-\mathcal{H}(r, t) \\
& \frac{\partial u}{\partial t}=-\mathcal{U}(r, t) \\
& \frac{\partial \tau_{r r}}{\partial t}=-\mathcal{R}(r, t) \\
& \frac{\partial \tau_{z z}}{\partial t}=-\mathcal{Z}(r, t) \\
& \frac{\partial \tau_{\theta \theta}}{\partial t}=-\mathcal{T}(r, t)
\end{aligned}
$$

where $\mathcal{R}, \mathcal{U}, \mathcal{R}, \mathcal{Z}$ and $\mathcal{T}$ are functions where the derivatives are computed in 
terms of mesh points values of the functions, as follows

$$
\begin{gathered}
\mathcal{H}=\hat{u}_{i} \frac{h_{i+1}-h_{i-1}}{d r_{i}+d r_{i+1}}+h_{i} \frac{u_{i}-u_{i-1}}{d r_{i}+d r_{i+1}}+\frac{\hat{u}_{i}}{r_{i}}, \\
\mathcal{U}=u_{i} \frac{u_{i+1}-u_{i-1}}{d r_{i-1 / 2}+d r_{i+1 / 2}}-3 S\left(\frac{\kappa_{i+1}-\kappa_{i}}{d r_{i}}\right)+\frac{3}{8} \frac{\hat{A}}{\hat{h}_{i}}\left(\frac{h_{i+1}-h_{i}}{d r_{i}}\right)+\frac{\hat{\tau}_{z z, i}}{\hat{h}_{i}}\left(\frac{h_{i+1}-h_{i}}{d r_{i}}\right) \\
\quad+\frac{\tau_{z z, i+1}-\tau_{z z, i}}{d r_{i}}-\frac{\hat{\tau}_{r r, i}}{r_{i+1 / 2}}-\frac{\hat{\tau}_{r r, i}}{\hat{h}_{i}}\left(\frac{h_{i+1}-h_{i}}{d r_{i}}\right)-\frac{\tau_{\theta \theta, i+1}-\tau_{\theta \theta, i}}{d r_{i}} \\
\quad-\frac{4}{r_{i+1 / 2}}\left(\frac{u_{i+1}-u_{i-1}}{d r_{i-1 / 2}+d r_{i+1 / 2}}\right)-\frac{4}{\hat{h}_{i}}\left(\frac{h_{i+1}-h_{i}}{d r_{i}}\right)\left(\frac{u_{i+1}-u_{i-1}}{d r_{i-1 / 2}+d r_{i+1 / 2}}\right) \\
+4\left(\frac{d r_{i+1 / 2} u_{i+1}+\left(d r_{i+1 / 2}+d r_{i+3 / 2}\right) u_{i}-d r_{i+3 / 2} u_{i-1}}{d r_{i+1 / 2}^{2} d r_{i+3 / 2}+d r_{i+1 / 2} d r_{i+3 / 2}^{2}}\right)+\frac{4 u_{i}}{r_{i+1 / 2}^{2}} \\
\left.\mathcal{R}=2 \frac{h_{i+1}-h_{i}}{d r_{i}}\right) \\
\mathcal{Z}=-2 \frac{\eta_{r}}{D e}\left(\frac{u_{i}-u_{i-1}}{d r_{i-1 / 2}}\right)-\frac{\tau_{r r, i}}{D e}-\hat{u}_{i}\left(\frac{\tau_{r r, i+1}-\tau_{r r, i-1}}{d r_{i+1}+d r_{i}}\right)+2 \tau_{r r, i}\left(\frac{u_{i}-u_{i-1}}{d r_{i-1 / 2}}\right) \\
\mathcal{T}=2 \frac{\eta_{r}}{D e} \frac{\hat{u}_{i}}{r_{i}}-\frac{\hat{\tau}_{\theta \theta, i}}{D e}-\hat{u}_{i}\left(\frac{\tau_{\theta \theta, i+1}-\tau_{\theta \theta, i-1}}{d r_{i+1}+d r_{i}}\right)+2 \tau_{r r, i} \frac{\hat{u}_{i}}{r_{i}} .
\end{gathered}
$$

here $\hat{u}, \hat{h}, \hat{\tau}_{r r}, \hat{\tau}_{z z}$ and $\hat{\tau}_{\theta \theta}$ are linear interpolated values of $u, h, \tau_{r r}, \tau_{z z}$ and $\tau_{\theta \theta}$ at $r_{i}$ and $r_{i+1 / 2}$, respectively.

\section{2}

\section{Crack-Nicholson method}

In computing unsteady flows, time derivatives also need to be discretized. A "time grid", with discrete points in time, can be used to perform the discretization using the finite difference approach. The major difference between space and time coordinates lies in the direction at which information is propagated; whereas a force at any spatial location may affect (in elliptic problems) all extension of the flow, forcing in a given instant affect only in the future - there is no backward influence. Therefore, unsteady flows are parabolic-like in time, which means that no conditions can be imposed on the solution (except at the boundaries) at any time after the initialization, which has a strong influence on the choice of solution strategy [28]. The time discretization was performed with the implicit Crank-Nicholson method, which is a second order accuracy of the trapezoidal rule method. This scheme is a mix of first-order explicit and implicit Euler method.

Performing the described space and time discretization schemes in Eqs. 
Eqs. 2-50 to 2-54 we have:

$$
\begin{aligned}
& \frac{h_{i}^{t}-h_{i}^{t-1}}{\Delta t}+\frac{1}{2} \mathcal{H}_{i}\left(\mathbf{h}^{t}, \mathbf{u}^{t}\right)+\frac{1}{2} \mathcal{H}_{i}\left(\mathbf{h}^{t-1}, \mathbf{u}^{t-1}\right)=0, \\
& \frac{u_{i}^{t}-u_{i}^{t-1}}{\Delta t}+\frac{1}{2} \mathcal{U}_{i}\left(\mathbf{h}^{t}, \mathbf{u}^{t}, \boldsymbol{\tau}_{\boldsymbol{r} r}{ }^{t}, \boldsymbol{\tau}_{\boldsymbol{z} z}{ }^{t}, \boldsymbol{\tau}_{\boldsymbol{\theta} \boldsymbol{\theta}}{ }^{t}\right)+\frac{1}{2} \mathcal{U}_{i}\left(\mathbf{h}^{t-1}, \mathbf{u}^{t-1}, \boldsymbol{\tau}_{\boldsymbol{r} \boldsymbol{r}}{ }^{t-1}, \boldsymbol{\tau}_{\boldsymbol{z} \boldsymbol{z}}{ }^{t-1}, \boldsymbol{\tau}_{\boldsymbol{\theta} \boldsymbol{\theta}}{ }^{t-1}\right)=0, \\
& \frac{\tau_{r, i}^{p, t}-\tau_{r r, i}^{p, t-1}}{\Delta t}+\frac{1}{2} \mathcal{R}_{i}\left(\mathbf{u}^{t}, \boldsymbol{\tau}_{\boldsymbol{r} r}{ }^{t}\right)+\frac{1}{2} \mathcal{R}_{i}\left(\mathbf{u}^{t-1}, \boldsymbol{\tau}_{\boldsymbol{r} \boldsymbol{r}}{ }^{t-1}\right)=0, \\
& \frac{\tau_{z z, i}^{p, t}-\tau_{z z, i}^{p, t-1}}{\Delta t}+\frac{1}{2} \mathcal{Z}_{i}\left(\mathbf{u}^{t}, \boldsymbol{\tau}_{\boldsymbol{z}}{ }^{t}\right)+\frac{1}{2} \mathcal{Z}_{i}\left(\mathbf{u}^{t-1}, \boldsymbol{\tau}_{\boldsymbol{z} \boldsymbol{z}}{ }^{t-1}\right)=0, \\
& \frac{\tau_{\theta \theta, i}^{p, t}-\tau_{\theta \theta, i}^{p, t-1}}{\Delta t}+\frac{1}{2} \mathcal{R}_{i}\left(\mathbf{u}^{t}, \boldsymbol{\tau}_{\boldsymbol{\theta} \boldsymbol{\theta}}{ }^{t}\right)+\frac{1}{2} \mathcal{T}_{i}\left(\mathbf{u}^{t-1}, \boldsymbol{\tau}_{\boldsymbol{\theta} \boldsymbol{\theta}}{ }^{t-1}\right)=0 .
\end{aligned}
$$

where $\mathbf{h}^{t}, \mathbf{u}^{t}, \boldsymbol{\tau}_{\boldsymbol{r} \boldsymbol{r}}{ }^{t}, \boldsymbol{\tau}_{\boldsymbol{z} \boldsymbol{z}}$ and $\boldsymbol{\tau}_{\boldsymbol{\theta} \boldsymbol{\theta}}{ }^{t}$ correspond to the solution vectors at time $t$, $\Delta t$ is the time step of the computation.

\section{3}

\section{Newton's method}

The discretization leads to a sparse set of coupled, nonlinear algebraic equations, that need to be solve at each time step. The method of choice to solve nonlinear system equations is Newton's method. The method consists of using the first two terms of multiple-variable Taylor series to linearize the functions.

Suppose $\mathbf{f}\left(x_{1}, x_{2}, \ldots x_{n}\right)$ is a set of non-linear equations that one need to find the roots, i.e, $\mathbf{f}\left(x_{1}, x_{2}, \ldots x_{n}\right)=0$, which can be approximated in term of first two terms of the Taylor series;

$$
f_{i}\left(x_{1}, x_{2}, \ldots, x_{n}\right) \approx f_{i}\left(x_{1}^{k}, x_{2}^{k}, \ldots, x_{n}^{k}\right)+\sum_{j=1}^{n}\left(x_{j}^{k+1}-x_{j}^{k}\right) \frac{\partial f_{i}\left(x_{1}^{k}, x_{2}^{k}, \ldots, x_{n}^{k}\right)}{\partial x_{j}}=0
$$

for $i=1,2 \ldots, n$. The system can be written as

$$
\sum_{j=1}^{n} A_{i, j}\left(x_{j}^{k+1}-x_{j}^{k}\right)=-f_{i}\left(x_{1}^{k}, x_{2}^{k}, \ldots, x_{n}^{k}\right)
$$

The term $f_{i}\left(x_{1}^{k}, x_{2}^{k}, \ldots, x_{n}^{k}\right)=R_{i}$ is the residual of the system and $A_{i, j}$ is the matrix of the system composed by the set of partial derivatives and also called Jacobian matrix:

$$
A_{i, j}=\frac{\partial f_{i}\left(x_{1}^{k}, x_{2}^{k}, \ldots, x_{n}^{k}\right)}{\partial x_{j}}
$$

For an initial estimative close to the solution, the method converges quadratically, i.e, the error at each iteration $k+1$ is proportional to the square 
of the error at iteration $k[28]$.

The Jacobian matrix was compute numerically via central-difference scheme

$$
J_{i, j}(\mathbf{x})=\frac{R_{i}\left(\mathbf{x}+\epsilon \mathbf{e}_{j}\right)-R_{i}\left(\mathbf{x}-\epsilon \mathbf{e}_{j}\right)}{2 \epsilon}
$$

where $\epsilon$ is a small positive number used to perturb the residual forward and backward. The tolerance on the L2-norm of the residual was set to $10^{-6}$.

\subsection{1}

\section{Validation}

In order to validate our numerical procedure, we compare our numerical prediction of the evolution of a planar perturbation in a stationary Newtonian liquid sheet with the benchmark results presented by Ida and Miksis [27] at $\frac{S}{\hat{A}}=\frac{1}{\pi^{2}}$. Figure 4.2 shows the free surface profile at different times. At this value of $\frac{S}{\hat{A}}$, the sheet is unstable and the perturbation grows until it pinches-off the liquid sheet. The numerical solution proceeds until $h(x=0, t)<0.01$. This time is considered the breakup time $t_{p}$. In the case shown in Fig.4.2, $t_{b}=1.52$. The time-evolution of the film thickness at $x=0$ is presented in Fig.4.3 for different number of discretization nodes. The plot also shows the predictions of Ida and Miksis as basis for comparison. For a number of nodes larger than 200 and with a parameter that controls the nodal points distribution $a=0.5$, the solution shows to be mesh independent and able to recover the literature data. 


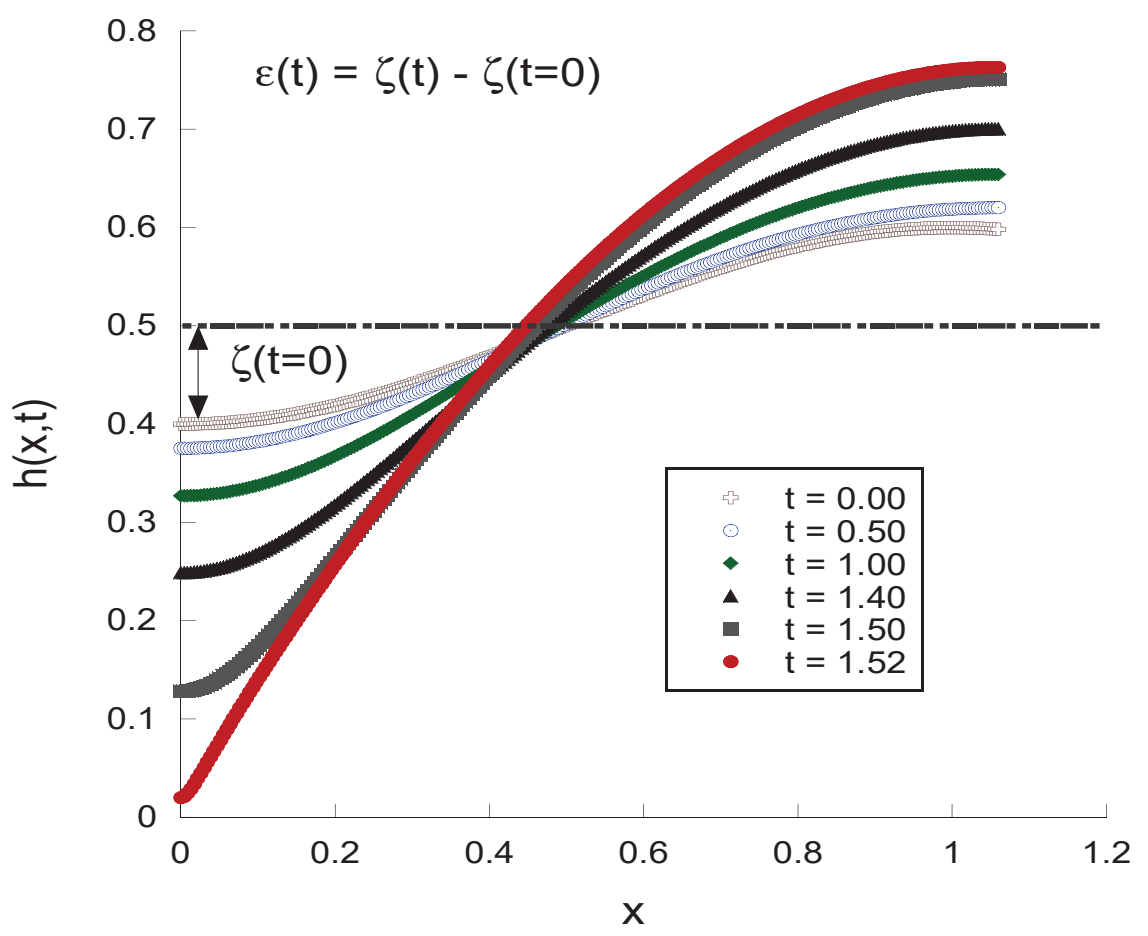

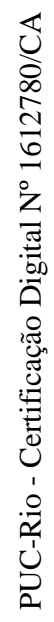

Figure 4.2: Thickness evolution profile of a Newtonian sheet under a planar perturbation at $S / A=1 / \pi^{2}$. The initial perturbation grows over time leading to a sheet rupture at $t=1.52$.

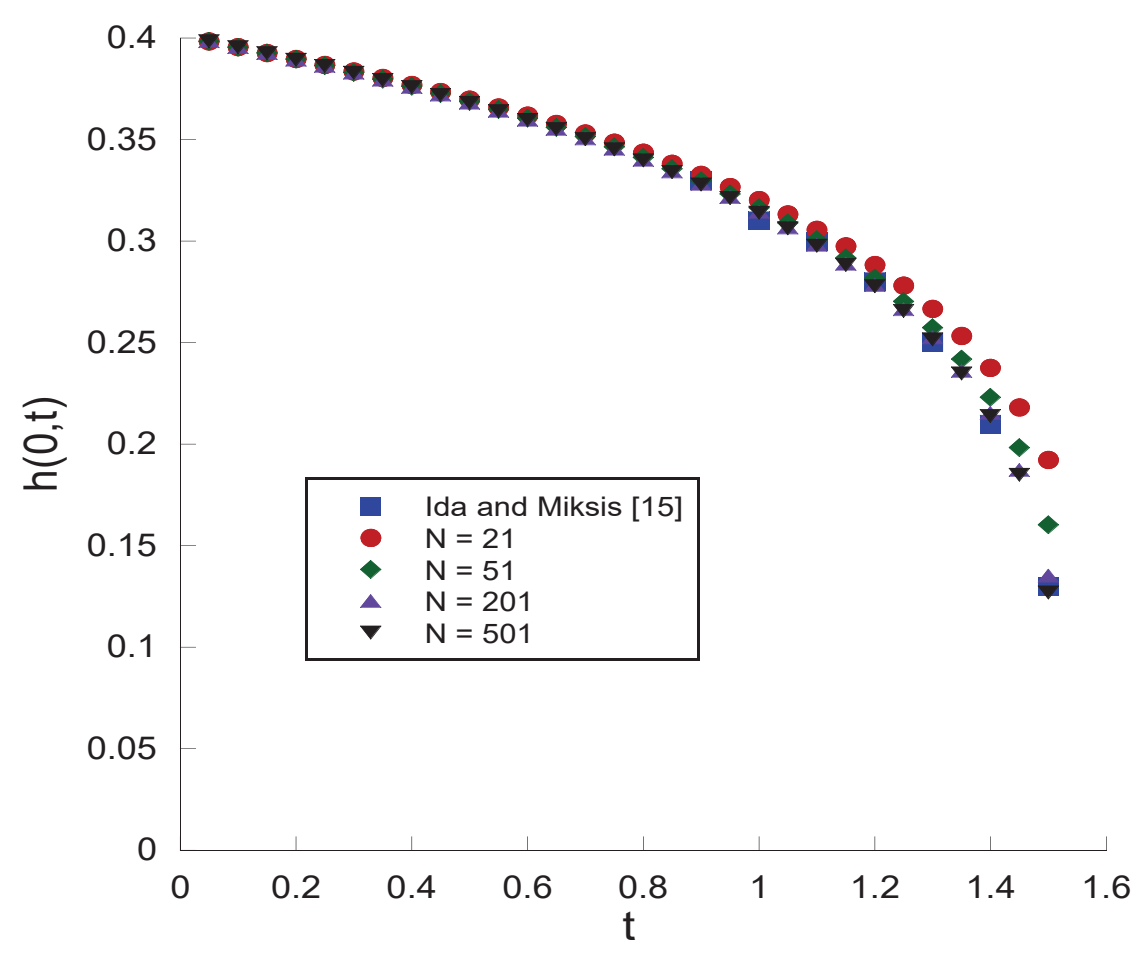

Figure 4.3: Evolution of sheet thickness at $x=0$ for different meshes at $S / A=1 / \pi^{2}$, The results recover predictions of Ida and Miksis [27]. 


\section{5 \\ Results and discussion}

This chapter presents the results of this work concerning to the analysis of time evolution of Newtonian and non-Newtonian thin liquids sheets under planar and axisymmetric perturbations. The dynamics can be represented in terms of the evolution of the film thickness variations with respect to the steady-state value at the perturbation point $x=0$ or $r=0$

$$
\zeta(t)=\bar{h}-h(t, r=0)
$$

where $\bar{h}$ is the steady-state uniform film thickness. The response of stationary liquid curtains under both type of disturbances with an initial amplitude equal to two percent of liquid sheet thickness, $\zeta_{0}=\zeta(t=0)=0.02$, are investigated.

Complementing the theoretical analysis developed in Chapter 3 , and looking to evaluate how precise is the linear stability analysis in predicting the stability criteria, Section 5.1 presents the results of numerical investigation of the stability criteria for Newtonian liquid sheet under planar and axysimmetric perturbations. The investigation is performed by varing the parameter $S / A$. Temporal evolution of perturbations are presented in terms of the initial perturbation, i.e $\varepsilon(t)=\zeta(t)-\zeta_{0}$, as illustrated in Fig. 4.2. Thus, if $\varepsilon(t)>0$, the perturbation grows over time, and the liquid curtain ruptures in a finite time.

Section 5.2 presents the results for non-Newtonian liquids. Generalized Newtonian Model (GNM) and Oldroyd-B model are used to describe different non-Newtonian behaviors. Based on those models, stability criteria and growth rate are analysed for planar and axisymmetric perturbations.

Finally, in section 5.3 we briefly discuss non-Newtonian effects on similarity solution.

\section{1}

\section{Numerical determination of stability criterion}

In chapter 3 we discussed stability criteria using linear stability analysis as proposed by Erneux and Davis [25]. Using this analysis we were able to find a critical ratio between capillary and van der Waals forces $S / A$ at which the 
flow changes from stable to unstable. Athought linear stability analysis can provide good insights about the flow behavior under certain flow conditions, one should carefully evaluate the results, due to the method limitations: results are based on linear equation, and negleting non-linear effects can lead to inaccurrate conclusion. In this section we numerically solve the full nonlinear lubrication equations derivated in Section 2 to examine the effect of the parameter $S / \hat{A}=3 \pi \sigma H^{4} / L^{2} A$ on the growth rate of the imposed disturbances in the early stage of the transient process. We propose a stability criterion based on these numerical predictions and compare it with the results from linear stability analysis.

For thin sheets of water-like liquids, $0.01 \pi^{2} \lesssim S / \hat{A} \lesssim 10 / \pi^{2}$, depending on the thickness $H$ and the wavelength $L$ of the perturbation. Based on these limits, we explored a range of values from $S / \hat{A}=0.1 \pi^{2}$ to $S / \hat{A}=3 / \pi^{2}$ in our study.

Figure 5.1 presents the value of $\varepsilon(t=1)$ as a function of $S / \hat{A}$ for both planar and axisymmetric perturbations. The value of $S / \hat{A}$ at which $\varepsilon(t=1)=0$ corresponds to the neutral stability point: $S / \hat{A}_{c} \approx 0.1442$ and $S / \hat{A}_{c} \approx 0.2026$, for axisymmetric and planar perturbations, respectively. These values match the critical parameters predicted by the stability analysis. The critical value of $S / \hat{A}$ is smaller for axisymmetric disturbances; the free surface curvature is higher in the axisymmetric case, leading to stronger capillary forces that stabilize the liquid sheet. 


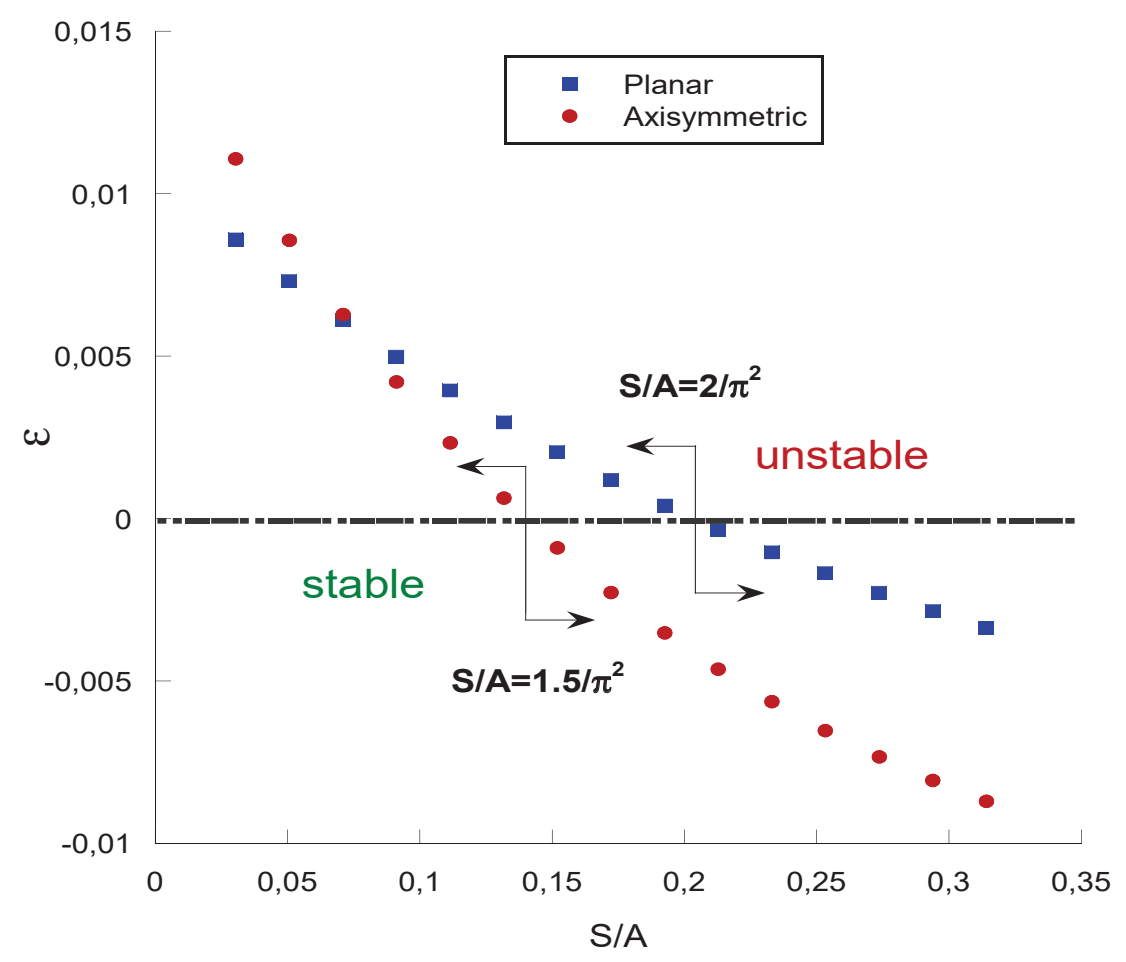

Figure 5.1: Planar and axisymmetric perturbations amplitude $\epsilon(t=1)=\zeta(t=$ 1) $-\zeta_{0}$ as a function of $S / \hat{A}$ for Newtonian liquids. $\epsilon>0$ corresponds to perturbations that grow and lead to sheet rupture in a finite time.

At low values of $S / \hat{A}$, e.g. $S / \hat{A} \lesssim 0.05$, the liquid curtain is unstable for both planar and axisymmetric disturbances, and the growth rate of axisymmetric perturbations is higher. Figure 5.2 presents the evolution of the film thickness at $x=0$ or $r=0$, for both planar and axisymmetric disturbances, for $S / \hat{A}=1 / 10 \pi^{2}$. The curtain rupture time is shorter for the axisymmetric disturbance. This may explain why thin liquid sheets pinch-off forming a circular hole not a straight line. 


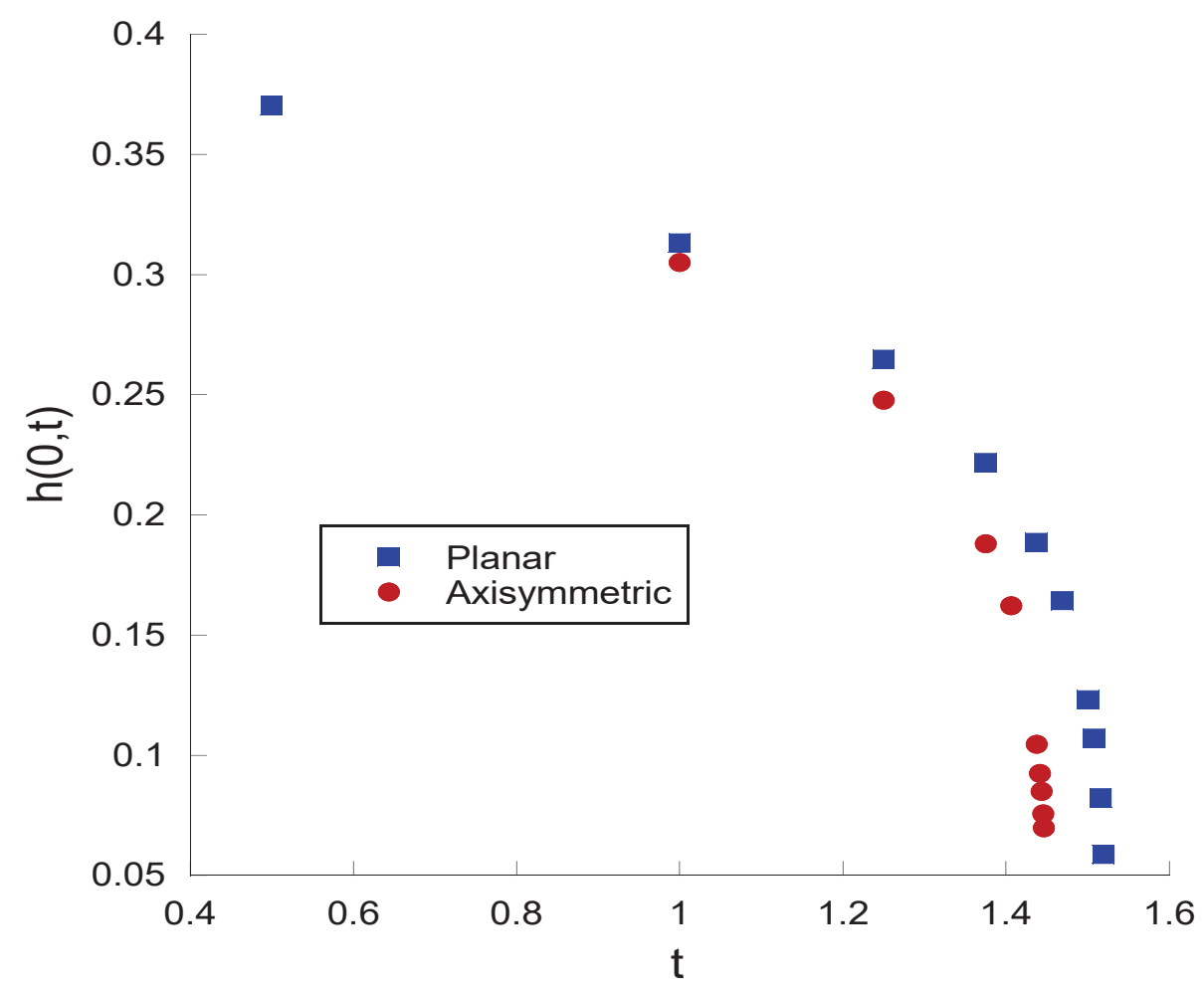

Figure 5.2: Evolution of sheet thickness at $x=0$ or $r=0$ at $S / \hat{A}=1 / 10 \pi^{2}$ for both planar and axisymmetric perturbation.

\section{2}

\section{Effect of non-Newtonian properties}

\subsection{1}

\section{Oldroyd-B model}

Oldroyd-B model is one of the simplest models that includes anisotropy and flow memory effects. The stress field is given by a transport equation that respect the frame-invariance principle. The stress equations of Oldroyd-B model must be solved coupled with radial velocity and thickness evolution. This section presents numerical results for an Oldroyd-B fluid as follow: first, an analysis of the effect of non-Newtonian properties in the stability criteria and perturbation growth rate, for planar and axisymmetric perturbation is presented, then, we investigate the influence of nonlinearities of the viscoelastic model in the rupture dynamics.

The dimensionless parameters that characterize the viscoelastic behavior of Oldroyd-B liquids are the Deborah number De and the polymer-to-solvent viscosity ratio $\eta_{r}=\eta_{p} / \eta_{s}$. Deborah number is defined as the ratio between the liquid relaxation time $t_{c}$, or the time that a given material takes to adjust 
to an applied stress and time of observation $t_{p}$, or characteristic timescale of an experiment or simulation: $D e=t_{c} / t_{p} . D e=0$ and $\eta_{r}=0$ recovers the Newtonian behavior.

In order to evaluate the effects of viscoelastic properties in the stability criteria, values of $\varepsilon(t=1)$ were calculated as a function of the parameter $S / \hat{A}$ at different $D e$ and $\eta_{r}$. Figures 5.3 for planar and 5.4 for axisymmetric perturbations show that, as revealed by the linear stability analysis, the viscoelastic behavior of the liquid does not affect the critical value of $S / \hat{A}$. However, it has a tremendous effect on the time-evolution of the imposed perturbation. The amplitude of the perturbation at $t=1$ falls significantly as Deborah number and polymer-to-solvent viscosity ratio rise.

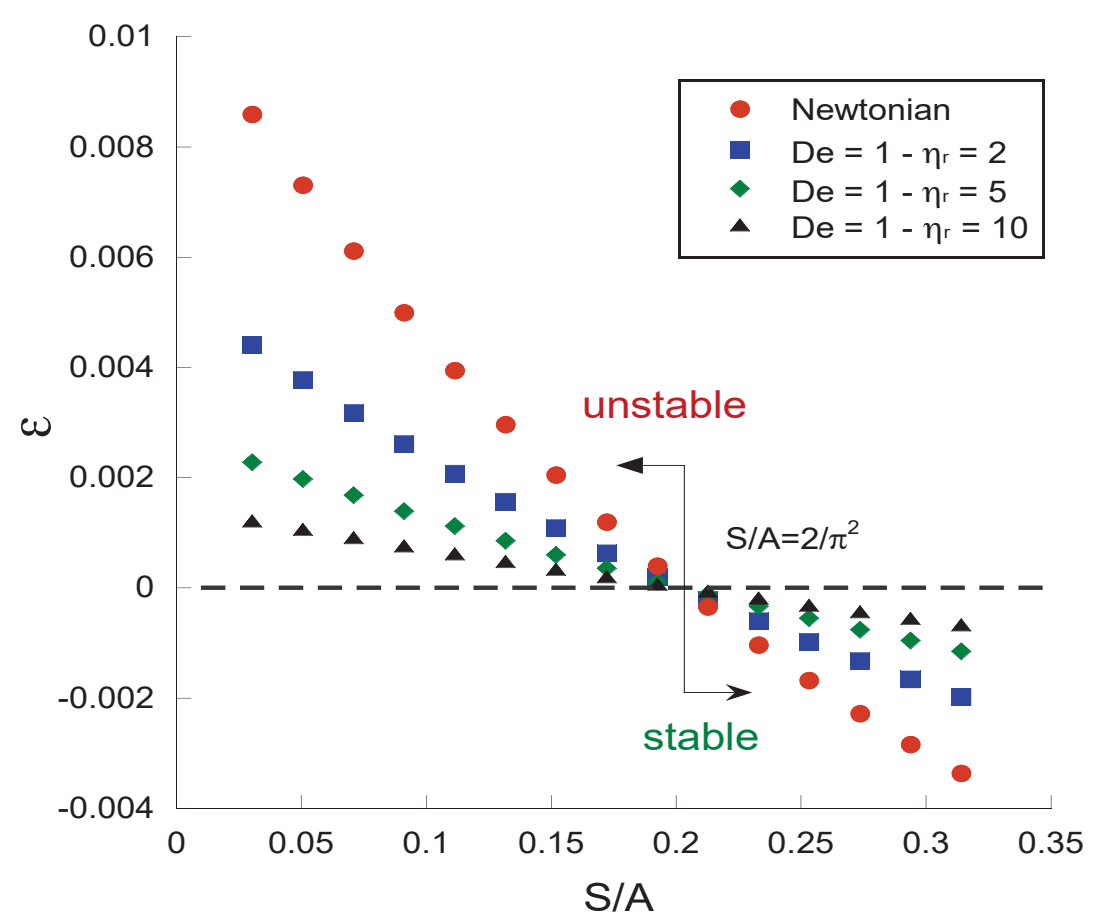

Figure 5.3: Planar perturbation amplitude $\epsilon(t=1)=\zeta(1)-\zeta_{0}$ as a function of $S / \hat{A}$ an liquid properties. $\epsilon>0$ corresponds to perturbations that grow and lead to sheet rupture. 


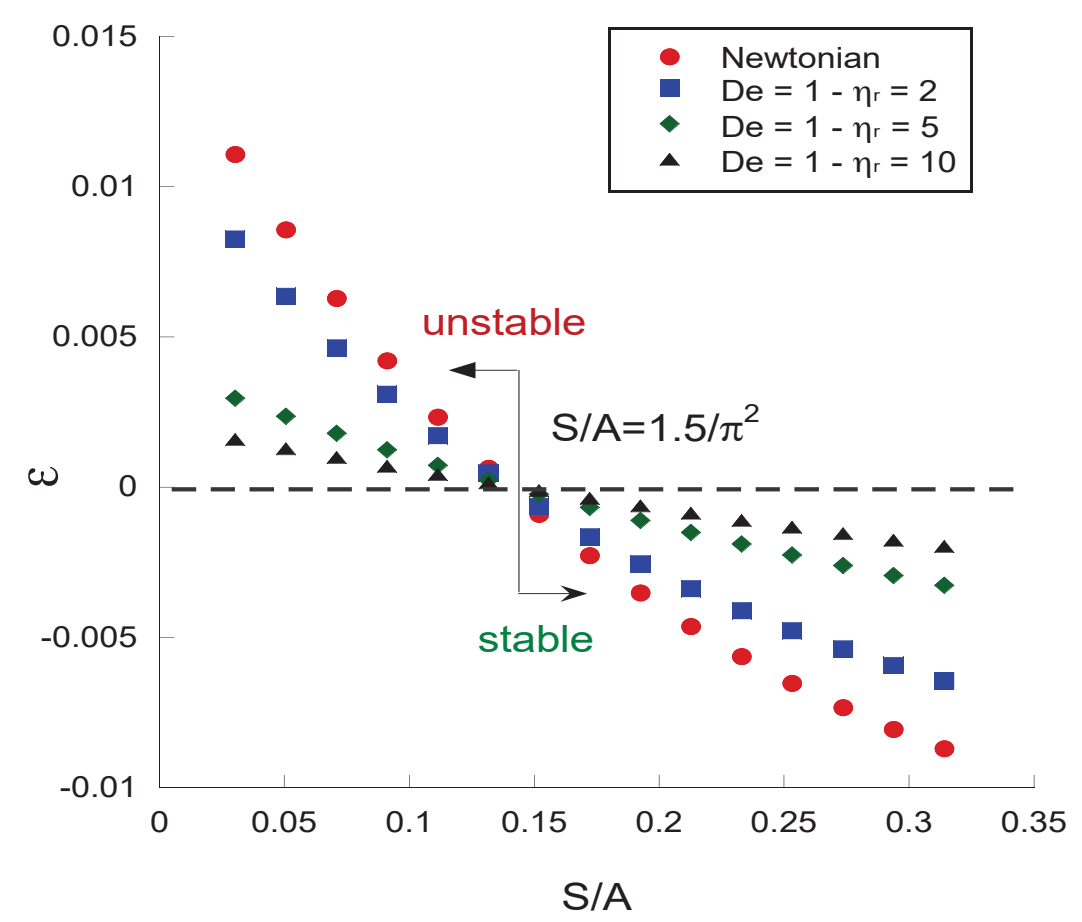

Figure 5.4: Axisymmetric perturbation amplitude $\epsilon(t=1)=\zeta(1)-\zeta_{0}$ as a function of $S / \hat{A}$ an liquid properties. $\epsilon>0$ corresponds to perturbations that grow and lead to sheet rupture

Figures 5.3 and 5.4 clearly show that elastic forces slow down the temporal growth of the perturbations. The slower growth rate of the perturbation leads to longer sheet rupture time, as it is clear in Fig. 5.5 The plot shows the predicted rupture time for planar perturbation as a function of the viscoelastic parameters at $S / \hat{A}=1 / \pi^{2}$. The rupture time at $D e=1$ and $\eta_{r}=10$ is close to $10 \times$ the rupture time of a Newtonian liquid sheet. In curtain coating applications, if the rupture time is longer than the residence time of the liquid in the curtain, the perturbation does not have enough time to grow and create a hole in the curtain. This may explain the experimental observations of Becerra and Carvalho [18] that the addition of high molecular weight polymer drastically delays the critical condition for curtain breakup. 


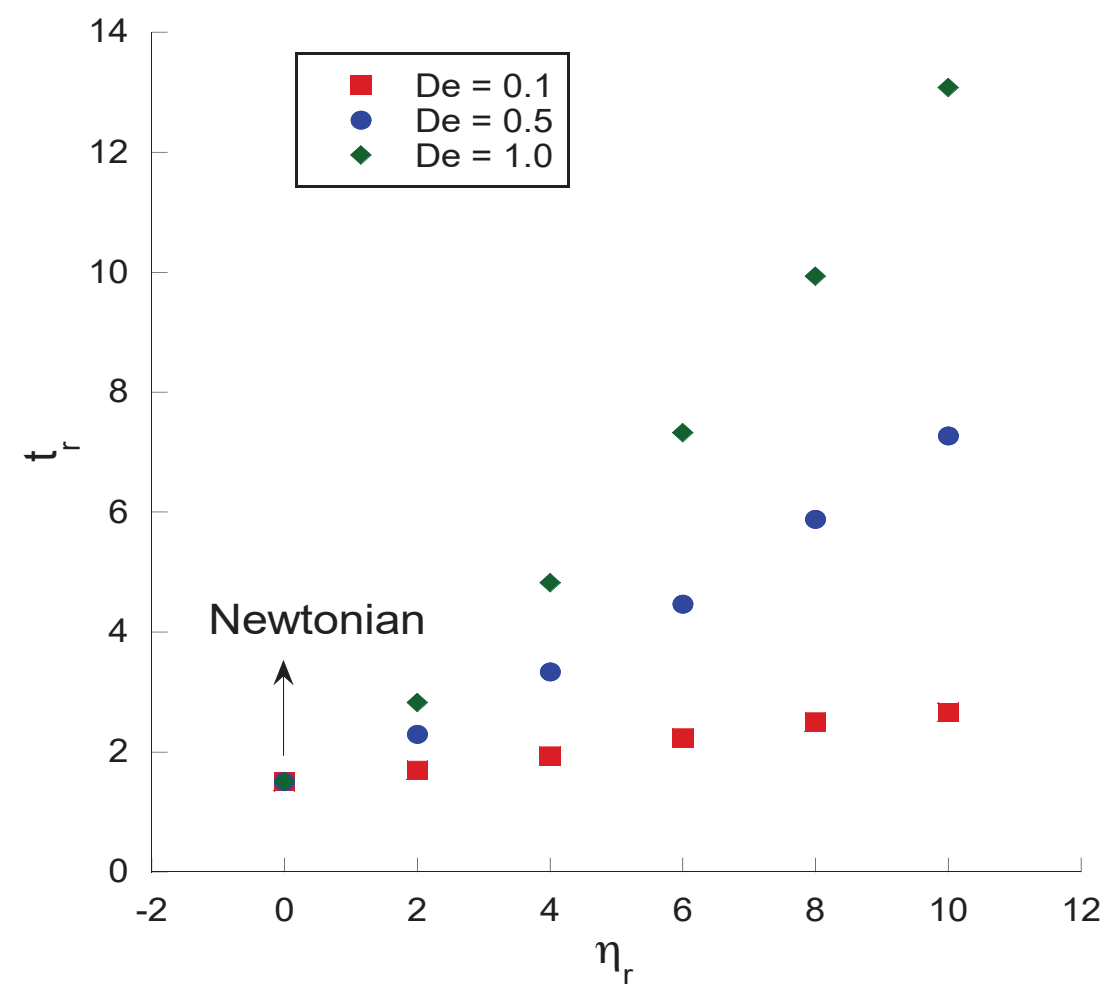

Figure 5.5: Sheet rupture time as a function of liquid properties for $S / \hat{A}=$ $1 / \pi^{2}$.

The delay in the rupture time can be clearly observed in the thickness evolution profiles at $t=1.5$ for different values of $\eta_{r}$, shown in Fig 5.6. While the curve representing the Newtonian fluid is almost at the point of sheet pinches-off, the curve with the strong viscoelastic properties $(D e=1$ and $\left.\eta_{r}=20\right)$ is still close to the amplitude of the imposed perturbance, which suggests a really slow growth of the perturbation. 


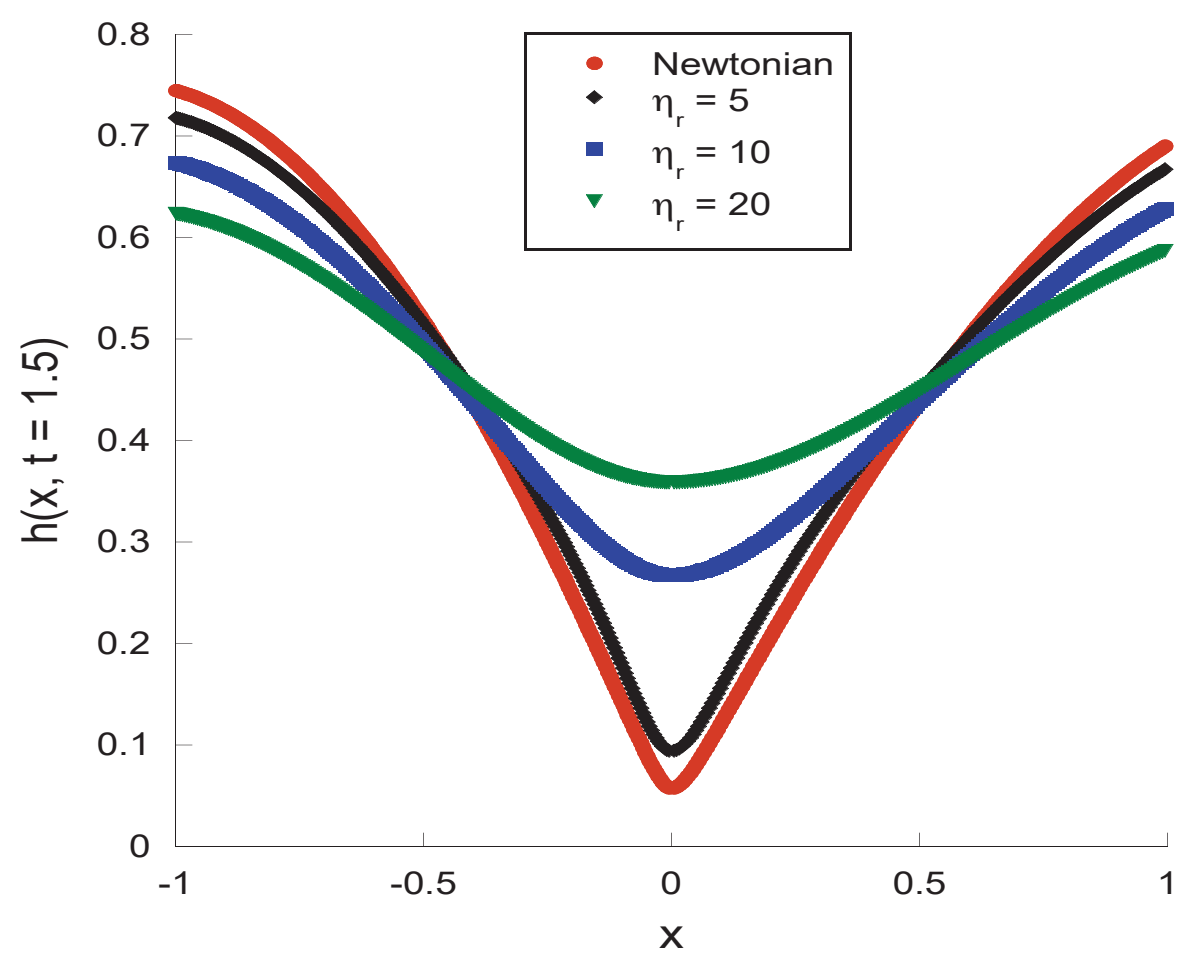

Figure 5.6: Thickness profile for $D e=1$ and different polymer-to-solvent viscosity ratio at $\mathrm{t}=1.5$.

An analysis of net force balance close to rupture time can provide interesting insights about the influence of viscoelasticity on the rupture dynamics. The terms associated with each force on the momentum conservation equation for planar and axisymmetric flow are shown below:

$$
\begin{aligned}
& \frac{\partial u}{\partial t}+\underbrace{u \frac{\partial u}{\partial x}}_{\text {Inertial }}-\underbrace{3 S \frac{\partial^{3} h}{\partial x^{3}}}_{\text {Capillary }}-\underbrace{\frac{3 \hat{A}}{8 h^{4}} \frac{\partial h}{\partial x}}_{\text {Van der Waals }}-\underbrace{\frac{4}{h} \frac{\partial h}{\partial x} \frac{\partial u}{\partial x}-4 \frac{\partial^{2} u}{\partial x^{2}}}_{\text {Viscous }}-\underbrace{\frac{\partial \tau_{x x}^{p}}{\partial x}-\frac{\tau_{x x}^{p}}{h} \frac{\partial h}{\partial x}}_{\mathbf{T}_{x x}^{p}} \\
& +\underbrace{\frac{\partial \tau_{y y}^{p}}{\partial x}+\frac{\tau_{y y}^{p}}{h} \frac{\partial h}{\partial x}}_{\mathrm{T}_{y y}^{p}}=0 \\
& \frac{\partial u}{\partial t}+\underbrace{u \frac{\partial u}{\partial r}}_{\text {Inertial }}-\underbrace{3 S\left(\frac{\partial^{3} h}{\partial r^{3}}+\frac{1}{r} \frac{\partial^{2} h}{\partial r^{2}}-\frac{1}{r^{2}} \frac{\partial h}{\partial r}\right)}_{\text {Capillary }}-\underbrace{\frac{4}{h}\left[\frac{\partial}{\partial r}\left(\frac{h}{r} \frac{\partial}{\partial r}(u r)\right)-\frac{u}{2 r} \frac{\partial h}{\partial r}\right]}_{\text {Viscous }} \\
& -\underbrace{\frac{3 \hat{A}}{8 h^{4}} \frac{\partial h}{\partial r}}_{\text {Van der Waals }}+\underbrace{\frac{1}{r} \frac{\partial}{\partial r}\left(h \tau_{z z}^{p}\right)}_{\mathrm{T}_{z z}^{p}}-\underbrace{\frac{1}{h r} \frac{\partial}{\partial r}\left(h r \tau_{r r}^{p}\right)}_{\mathrm{T}_{r r}^{p}}+\underbrace{\frac{\tau_{\theta \theta}^{p}}{r}}_{\mathrm{T}_{\theta \theta}^{p}}=0
\end{aligned}
$$

The values of each force along the liquid sheet in the vicinity of the pinch-off point, for a Newtonian liquid, when thickness reaches $h(x=0, t)=0.025$ for 
planar and $h(r=0, t)=0.075$ for axisymmetric are shown in Figs. 5.7 and 5.8. As proposed by Ida and Mikis [27] and Vaynblat et al. [43], tension profiles suggest that viscous and van der Waals force dominate the dynamics in the final stage of the rupture process in both cases. Figure 5.9 presents tension profiles for a planar perturbation type in very viscoelastic regime, with $D e=1$ and $\eta_{r}=10$. One can observe that elastic force rises in the flow, and contributes with viscous forces to hindering the perturbation growth. This behavior can explain the increase in the rupture time observed in Fig. 5.5.

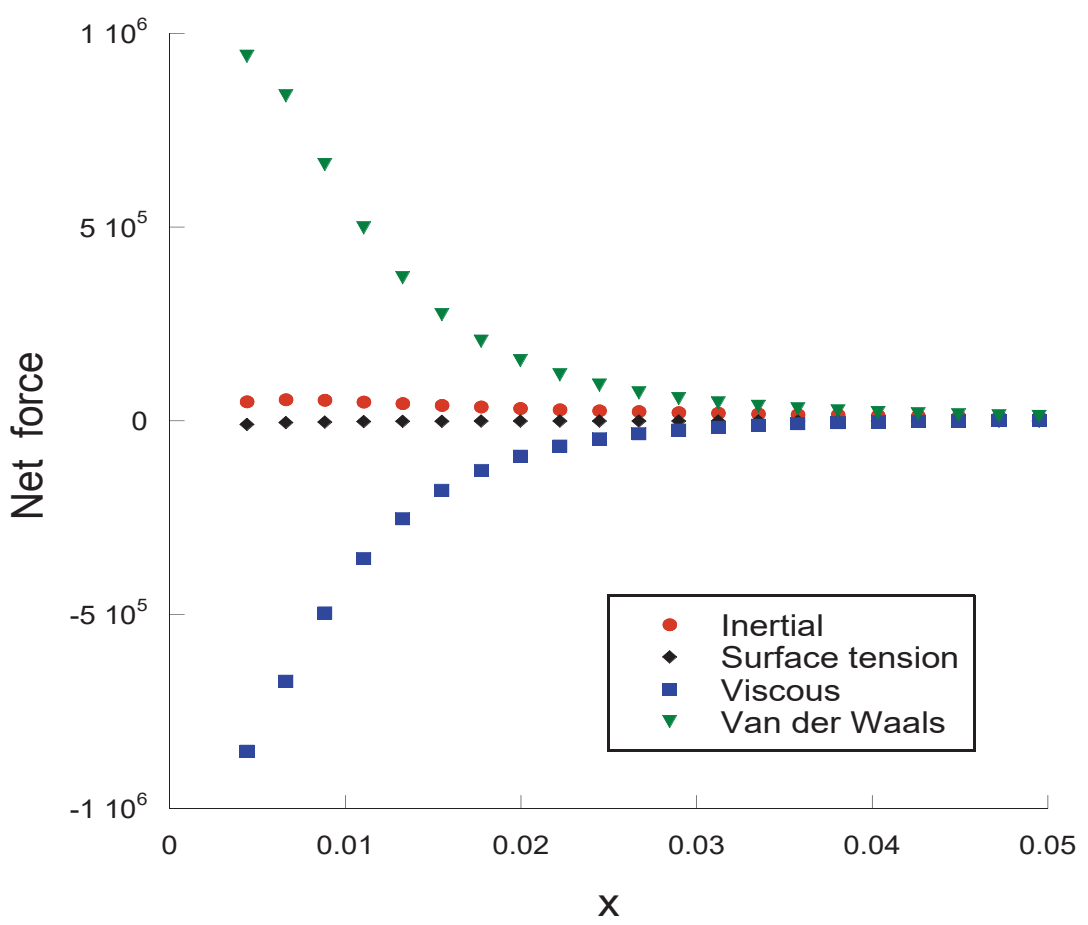

Figure 5.7: Net force profile of Newtonian fluid under a planar perturbation in the vicinity of rupture point when thickness reaches $5 \%$ of the undisturbed film thickness. 


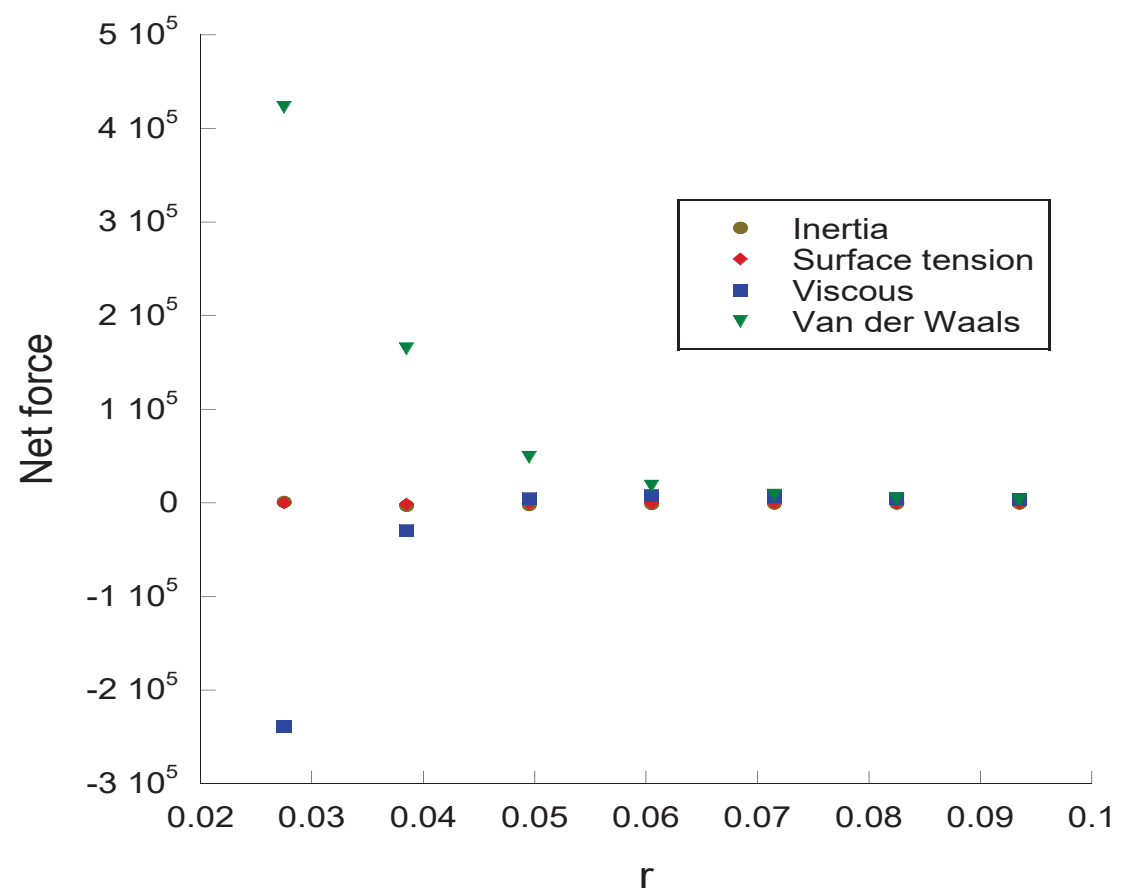

Figure 5.8: Net force profile of Newtonian fluid under an axisymmetric perturbation in the vicinity of rupture point when thickness reaches $15 \%$ of the undisturbed film thickness.

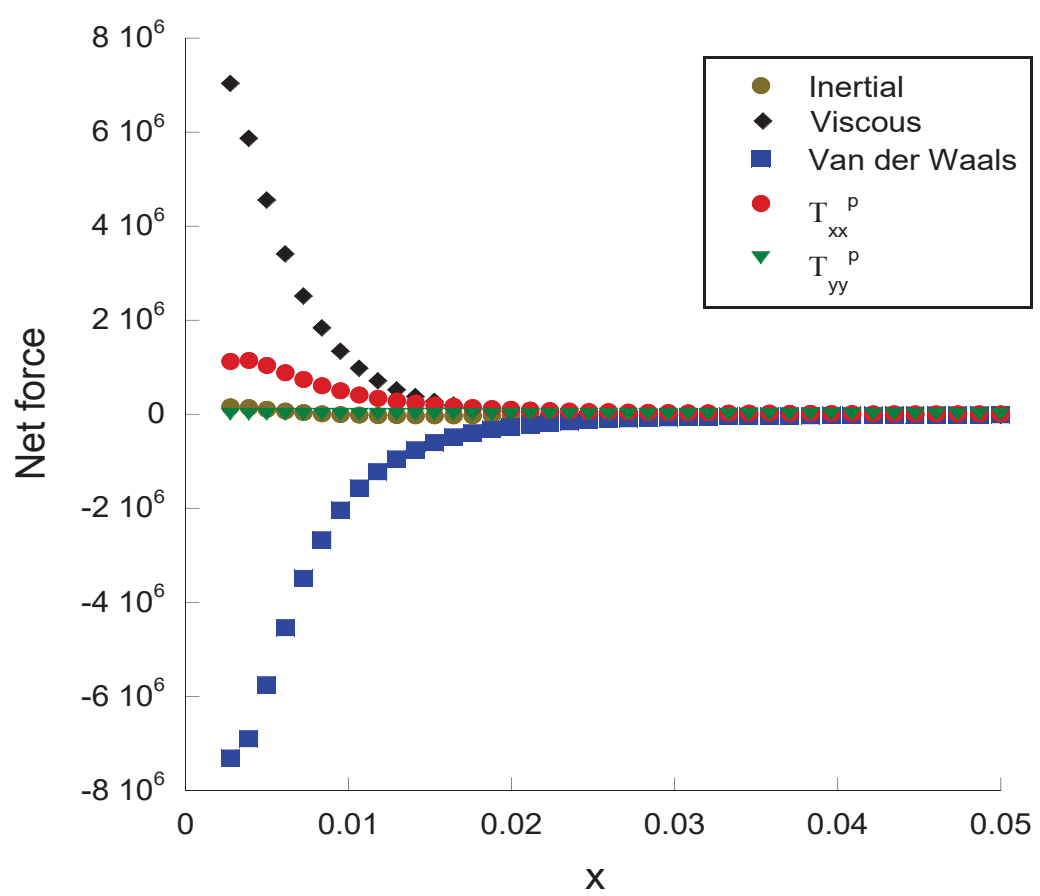

Figure 5.9: Net force profile Oldroyd fluid for a $D e=1$ and $\eta_{r}=10$ under a planar perturbation in the vicinity of rupture point when thickness reaches $5 \%$ of the undisturbed film thickness. 
Figure 5.10 shows the velocities profiles for Newtonian and viscoelastic sheets subjected to planar perturbations. It is possible to observe that the rupture dynamics is concentrated in the vicinity of the pinch-off region, while the far-field flow remains undisturbed. Moreover, the results show that, althought the velocity profiles have a similar behavior, the Newtonian liquid shows a more extended region influenced by the rupture dynamics as well as a higher value of the radial velocity.

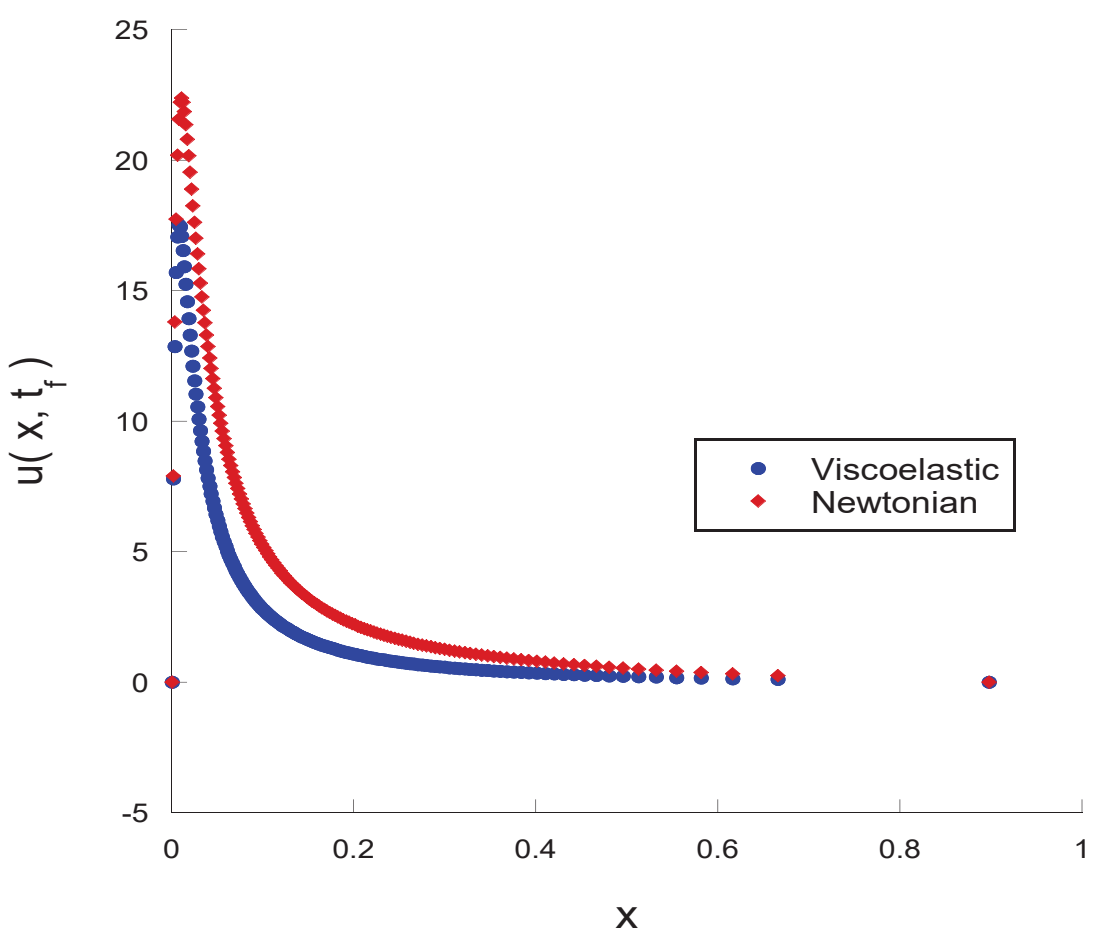

Figure 5.10: Velocity profile for Newtonian and Oldroyd-B fluids for planar perturbation type. Viscoelastic parameters were set to be $D e=1$ and $\eta_{r}=10$. And $t_{f}$ represents the moment when final thickness reaches $5 \%$ of the undisturbed film thickness.

\section{3}

\section{Similarity analysis}

The symmetry of certain systems allows properties to remain unchanged under certain transformations. Scale symmetry happens when a physical phenomenon is invariant with respect to changes in units of measurement or with respect to the scaling of quantities themselves. Self-similarity results when the symmetry of a physical problem leads to the reduction in the number of independent variables, thus, achieving considerable simplification of the problem [52]. In general, self-similar behavior occurs in the intermediate asymptotics of physical phenomena, when initial or boundary conditions are 
no longer relevant. The characteristic of the passage to the limit that leads to intermediate asymptotic of a given problem allows to classify similarity solutions into two types; first-type is found when the passage to the limit is regular, in that case, self-similar variables can be obtained by dimensional analysis or symmetry consideration. Second-type results when the passage to the limit is irregular that requires following the evolution of the solution until it passes into its self-similarity asymptotics [52]. Long-wavelength models for van der Waals driven rupture of a free thin viscous sheet give rise to families of first-type similarity solution which has been a widely explored subject in literature for Newtonian [51, 27] and non-Newtonian fluids [44].

Studies of self-similarity solution involving non-Newtonian fluids were presented by Thete et al [44]. Where, based on numerical solutions, they examined the self-similarity evolution of a power-law liquid film, evaluated the scaling exponent governing the time evolution of the film thickness and constructed similarity profiles for the interface shape. For power-law fluids, the governing equations remain similar to Newtonian fluid equations. However, Oldroyd-B fluids include an extra tensorial transport equation, which further increase the difficulty of any kind of analysis or analytical solution. Thus, this section is not concerned with determining a formal self-similarity solution for viscoelastic fluids, but, to analyze how the self-similarity solution is affected by viscoelastic properties.

Approaching to rupture time, one might expect that the length and time scales of motion in the vicinity of the rupture point are an order of magnitude smaller than those in the far-field. Hence, self-similar solutions, for a Newtonian fluid can be written in the form of [27]:

$$
h(x, t)=\tau^{\alpha} H(\xi) \quad u(x, t)=\tau^{\Gamma} U(\xi),
$$

where $\tau=t_{r}-t\left(t_{r}\right.$ is the rupture rime) and $\xi=x \tau^{-\beta}$.

Ida and Miksis [27] and Vaynblat et al [43] have shown that for Newtonian liquids, the force balance sets the exponents of the similarity solution to:

$$
\alpha=\frac{1}{3}, \quad \beta=\frac{1}{2} \quad \text { and } \quad \Gamma=-\frac{1}{2}
$$

The effect of shear-sensitive viscosity on the similarity solution was investigated by Thete et al [44] for power-law liquids. The study proposed that similarity exponents are modified due the non-Newtonian effects as following:

$$
\alpha=\frac{n}{3}, \quad \beta=1-\frac{n}{2} \quad \text { and } \quad \Gamma=-\frac{n}{2},
$$


where $n$ is the power-law exponent, $n<1$ for shear-thinning liquids. When $n=1$, the solution corresponds to a Newtonian fluid and recovers Vaynblat's results.

To show the effect of viscoelastic forces in the dynamics of the liquid sheet rupture, Fig. 5.11 presents the logarithm of the film thickness at $x=0$ as a function of the logarithm of $\tau=t_{r}-t$ at $S / \hat{A}=1 / \pi^{2}$ for both Newtonian and viscoelastic liquids ( $D e=1$ and $\eta_{r}=20$ ). Near the rupture time, e.g. at low values of $\tau$, the numerical predictions are well fitted by a straight line in Fig.5.11, indicating that the solution follows the form $h(x, t) \approx \tau^{\alpha}$. The slope of the Newtonian results is $\alpha=0.31$, which is close to the similarity exponent $\alpha=1 / 3$ presented in the literature $[27,43]$. For the viscoelastic solution, the slope is $\alpha=0.23$, indicating that the viscoelastic forces slow down the growth of the perturbation.

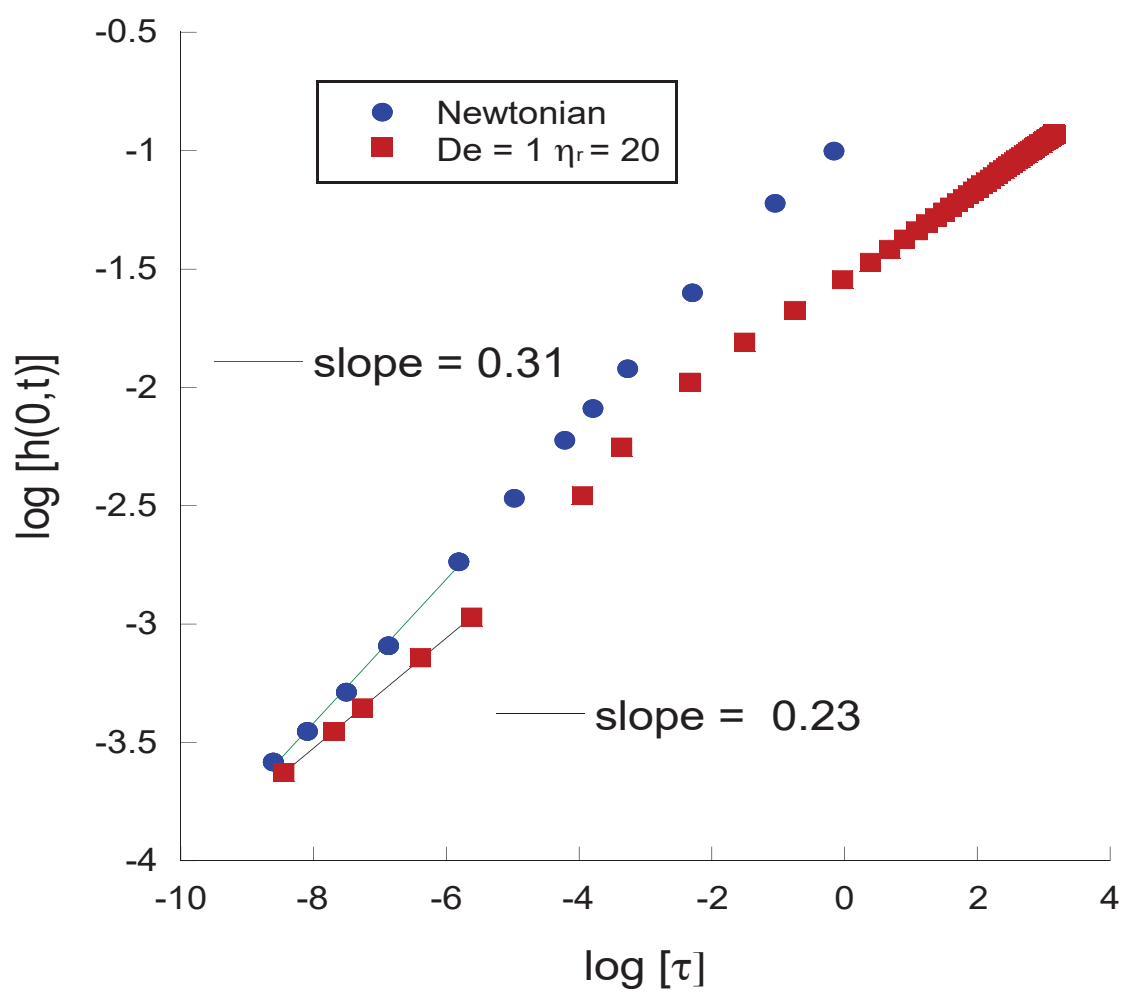

Figure 5.11: Dynamics of film thickness at $x=0$ close to the rupture time $t_{b}$. The sheet thickness follows the form $h \approx\left(t_{b}-t\right)^{\alpha}$.

\subsection{1}

\section{Generalized Newtonian model}

Generalized Newtonian model (GNM) may describe some aspects of viscoelastic behavior by allowing viscosity to change with rate-of-extension. The dimensionless parameters that characterize the viscoelastic behavior of 
GNM liquids are the Deborah number $D e$, which sets the cross-over extansional rate, and the infinity-extensional-viscosity ratio $\eta_{r}=\eta_{\infty} / \eta_{0}$.

For a planar flow, the dimensionless expression for viscosity is:

$$
\eta=\eta_{r}+\left(1-\eta_{r}\right)\left[1+\left(D e \frac{\partial u}{\partial x}\right)^{2}\right]^{\frac{n-1}{2}}
$$

where $n=0.5$. Setting $D e=1$, then $\eta=1$, and conservation equations recover the Newtonian behavior.

Figures 5.12 and 5.13 show that, differently from Oldroyd-B model, in GNM model non-Newtonian effects are more pronounced for high values of $\eta_{r}$ and $D e$, and in the latter stage of rupture. In this sense, the stability criterion and growth rate of perturbation in the early stage of the rupture process matches with the Newtonian model regardless of the non-Newtonian parameters.

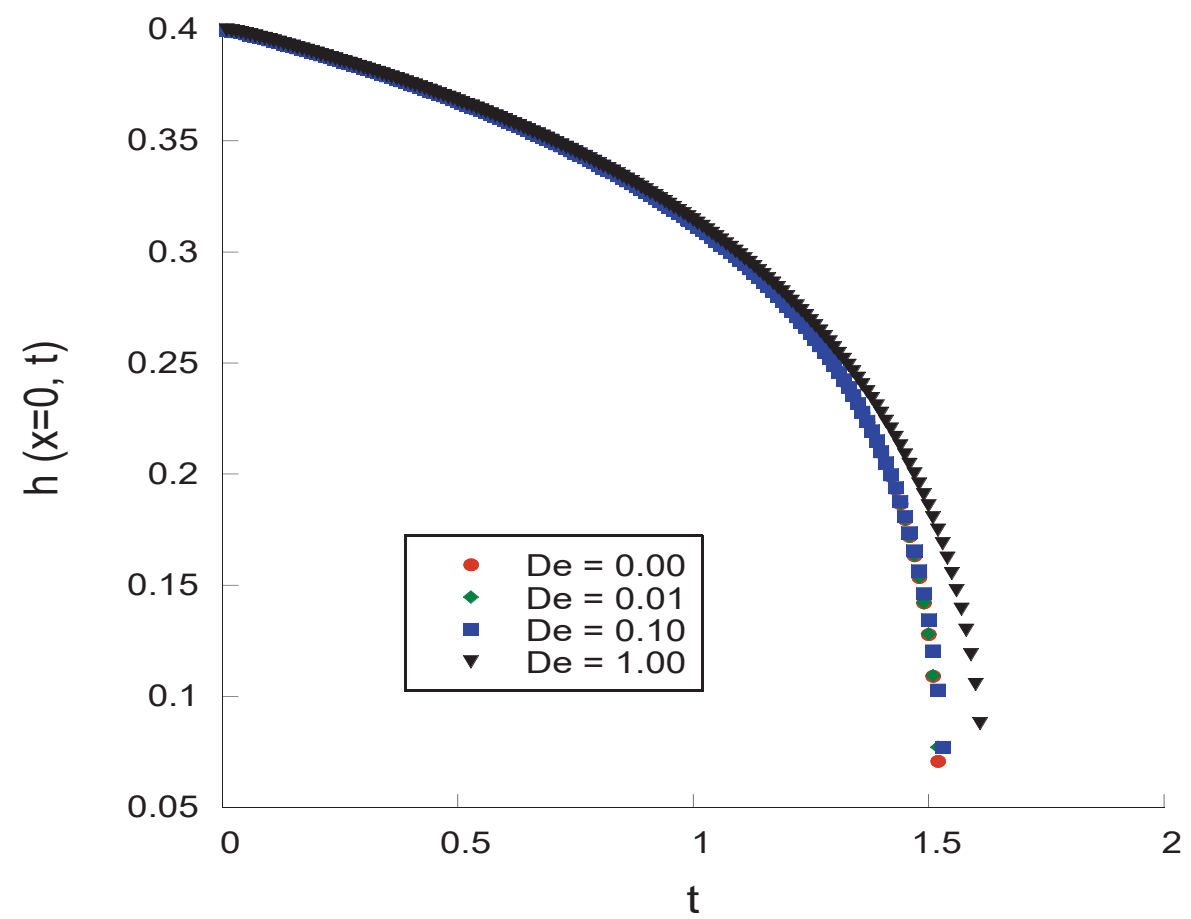

Figure 5.12: Temporal thickness evolution for Newtonian and GNM models for $\eta_{r}=2$. Profiles show that non-Newtonian effects are barely perceptible for low values of $\eta_{r}$. 


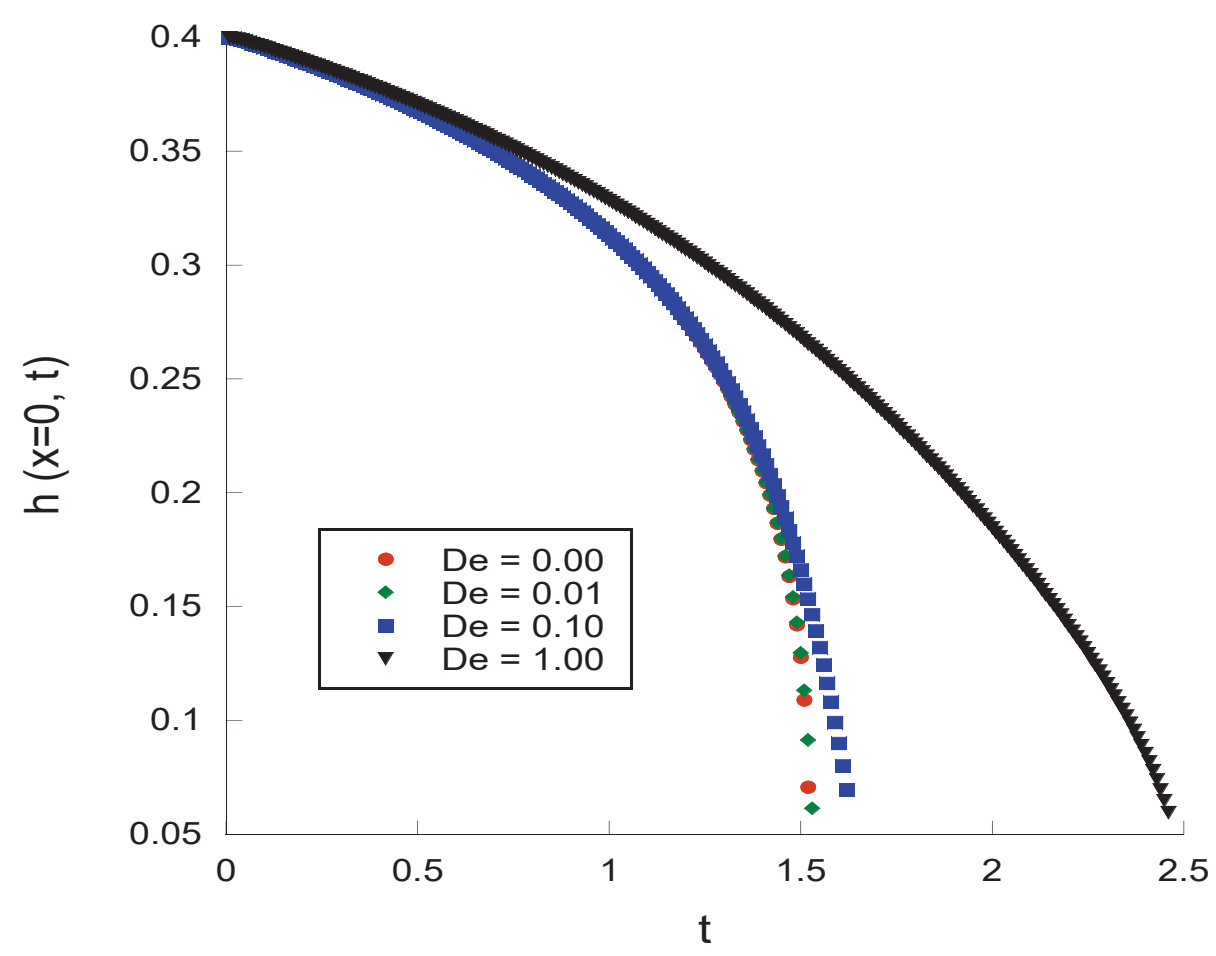

Figure 5.13: Temporal thickness evolution for Newtonian and GNM models for $\eta_{r}=20$. Profiles show that non-Newtonian effects are more pronounced in the latter stage of rupture. Substantially effects on the rupture time are only observed for a high Deborah number.

Figures 5.14 and 5.15 compare different net force profiles for GNM ( $D e=1$ and $\left.\eta_{r}=10\right)$ and Newtonian models under a planar perturbation. For a time at which the film thickness reaches $5 \%$ of the undistubed film thickness, one can observe that viscous and van der Waals forces are still dominant close to the rupture time. A curious effect can be observed here; in longitudinal extension, the region affected by the rupture dynamics is much larger for GNM fluid than for a Newtonian fluid. The same behavior can be observed in Fig. 5.16 for velocities profile. This behavior can be explained by the increase in viscosity close to rupture point, due to a large rate-of-extension in this region, as can be observed in Fig. 5.17. 


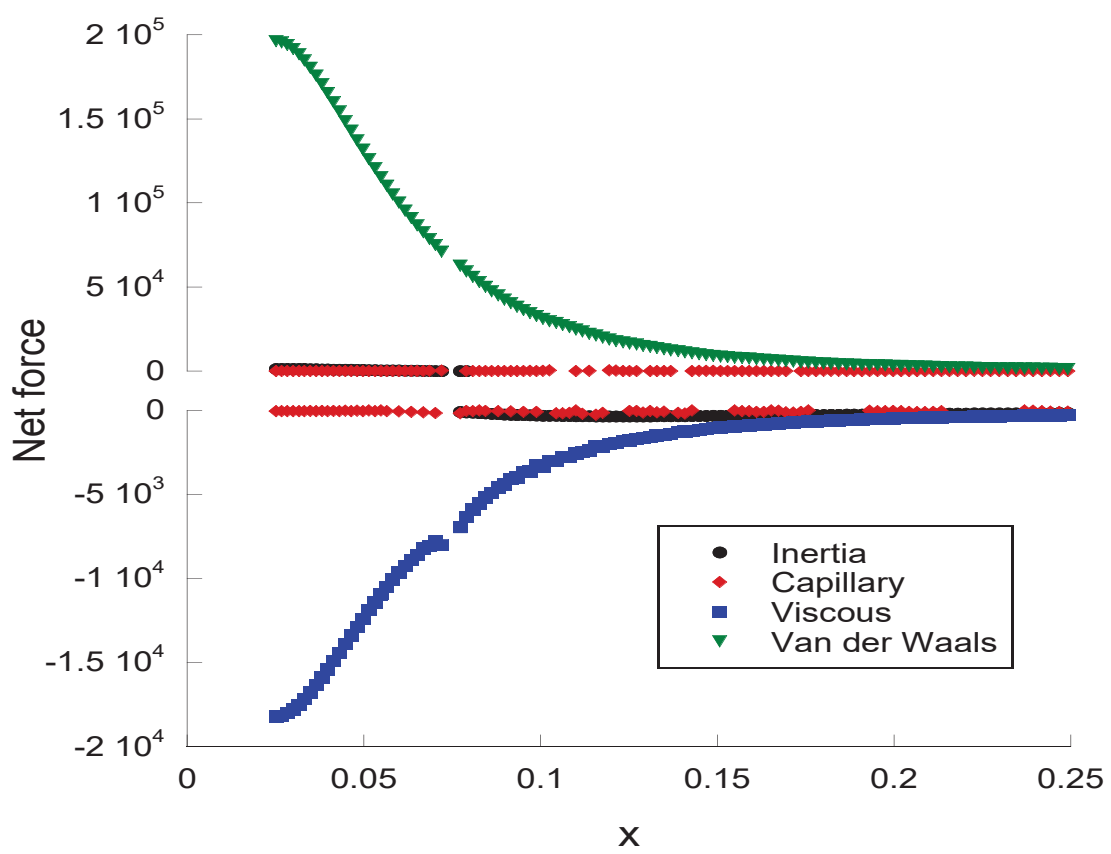

Figure 5.14: Net force profiles of GNM fluid for a $D e=1$ and $\eta_{r}=10$ under

a planar perturbation in the vicinity of rupture point when thickness reaches $5 \%$ of the undisturbed film thickness.

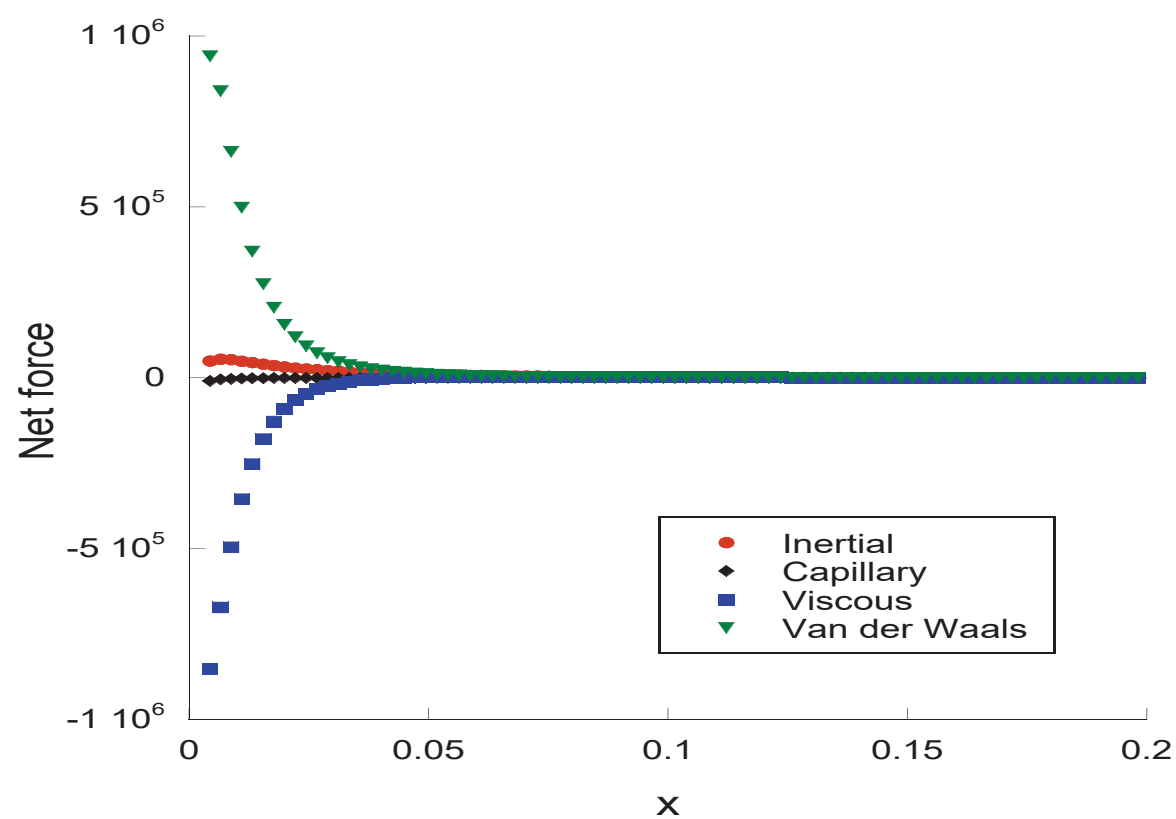

Figure 5.15: Net force profiles of Newtonian fluid under a planar perturbation in the vicinity of rupture point when thickness reaches $5 \%$ of the undisturbed film thickness. 


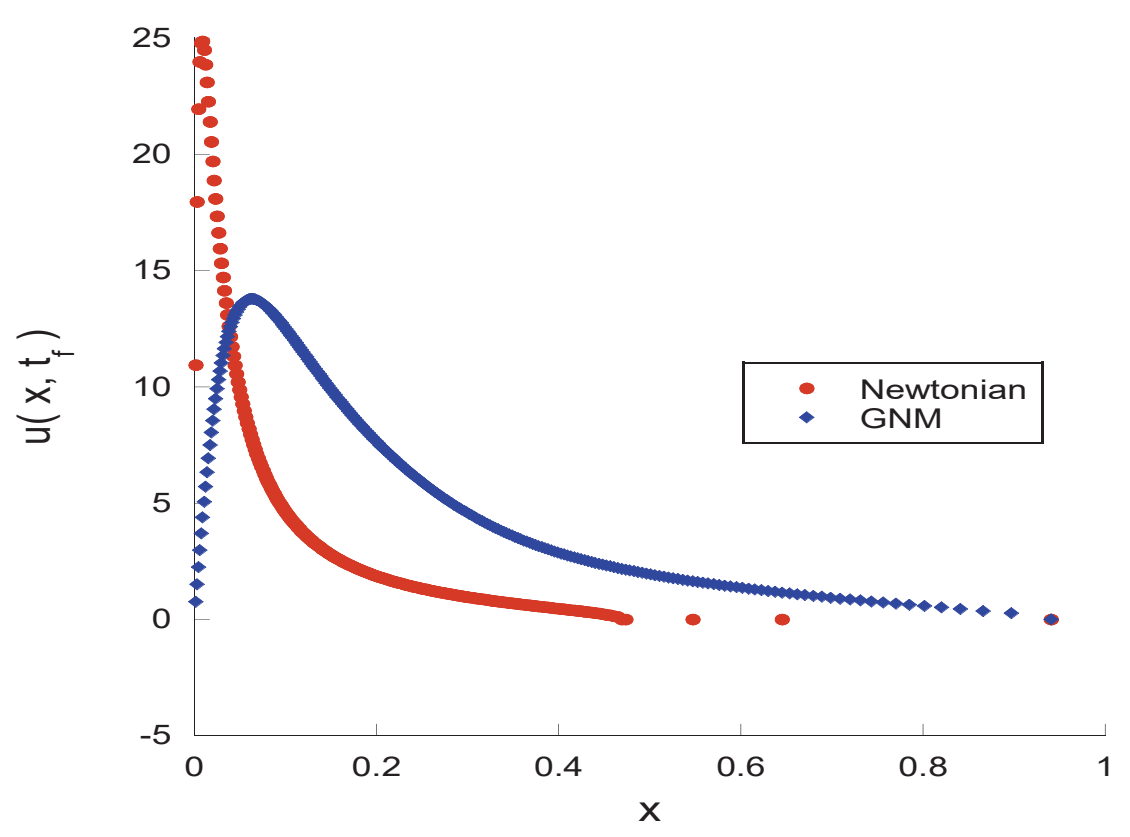

Figure 5.16: Velocity profile for Newtonian and GNM fluids for planar perturbation type. Viscoelastic parameters were set to be $D e=1$ and $\eta_{r}=10$. And $t_{f}$ represents the moment when final thickness reaches $5 \%$ of the undisturbed film thickness.

Figure 5.17 shows the viscosity ratio profile along the axial direction for $D e=1$ and $\eta_{r}=10$. It is possible to observe that as the rupture approaches, the viscosity increases in all the longitudinal extension.

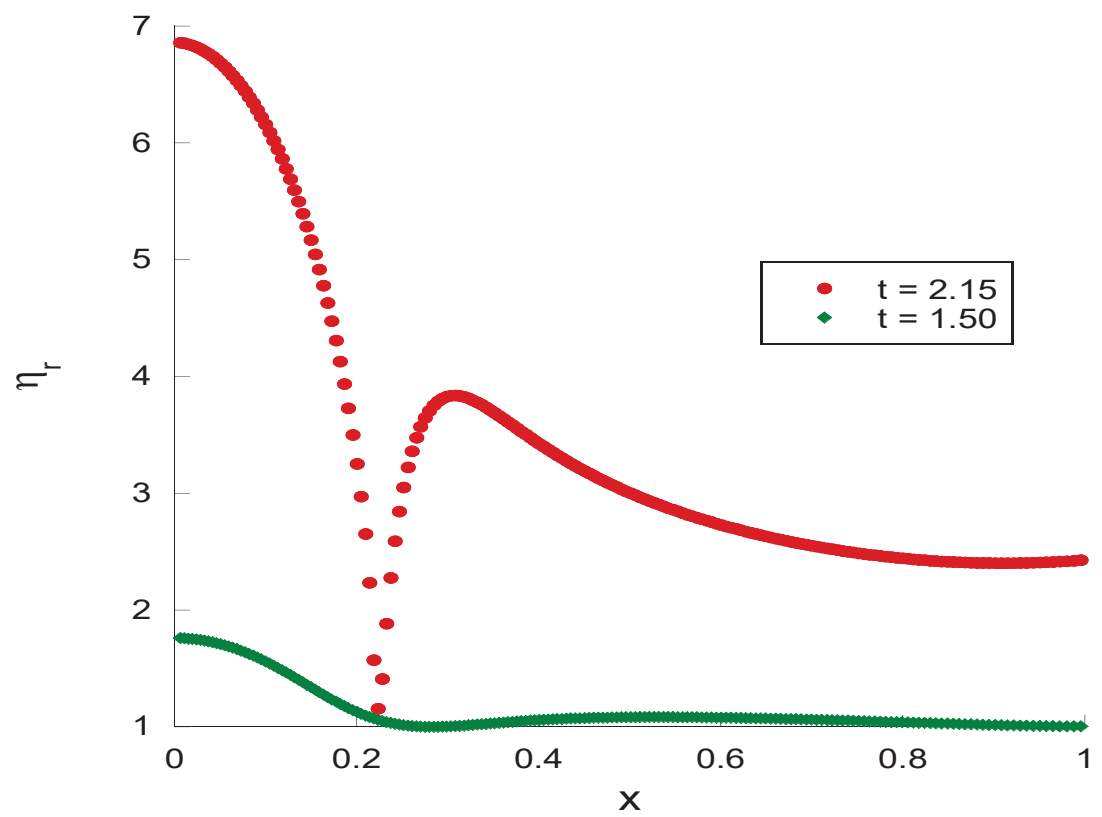

Figure 5.17: Viscosity ratio profile along the axil direction for $D e=1$ and $\eta_{r}=10$ in an intermediate time $t=1.52$ and in the final time $t=2.15$. 


\section{Part II}

\section{Sheet retraction}




\section{6 \\ Mathematical modeling}

This chapter presents the mathematical formulation used to describe the sheet rectraction, including momentum conservation equations, stress tensor model, boundary and initial conditions.

\section{1}

\section{Conservations equations}

Similarly to section 2, flow is considered two dimensional and isothermal. Fluid is incompressible and bounded by a passive gas phase. The consevation equations include continuity and Cauchy equation of motion, namely

$$
\begin{aligned}
& \nabla \cdot \mathbf{u}=0, \\
& \rho \frac{D \mathbf{u}}{D t}=\nabla \cdot \mathbf{T},
\end{aligned}
$$

where $\rho$ is the liquid density, $\mathbf{u}$ is the velocity field and $\mathbf{T}=p \mathbf{I}+\boldsymbol{\tau}$ is the stress tensor, divided into an isotropic and deviatoric component. In order to complement the work presented ealier in Part I, and study the retraction process of a ruptured non Newtonian sheet, the stress tensor is also defined by both models described in Section 2. The GNM model includes rate-of-extension dependent viscosity

$$
\eta(\dot{\epsilon})=\eta_{\infty}+\left(\eta_{0}-\eta_{\infty}\right)\left(1+(\lambda \dot{\epsilon})^{2}\right)^{\frac{n-1}{a}} .
$$

For the Oldroyd-B model the deviatoric stress tensor is divided into two terms: solvent stress $\tau^{s}$ and polymeric stress $\tau^{p}$. The solvent stress is described by the Newton's of viscosity:

$$
\tau^{p}=\eta_{s} \dot{\gamma}
$$

The polymeric stress is given by the upper-convected Maxwell model,

$$
\tau^{p}+\lambda \stackrel{\nabla}{\tau^{p}}=\eta_{p} \dot{\gamma}
$$


where $\stackrel{\nabla}{\tau^{p}}$ is the convected derivative:

$$
\stackrel{\nabla}{\boldsymbol{\tau}}=\frac{D}{D t} \boldsymbol{\tau}-(\boldsymbol{\tau} \cdot \boldsymbol{\nabla} \boldsymbol{u})^{T}-(\boldsymbol{\tau} \cdot \boldsymbol{\nabla} \boldsymbol{u})
$$

\section{2}

\section{Boundary condition}

Equations 6-1 and 6-2 are solved using Cartesian coordinate system for straight line retraction and cylindrical coordinate system for circular hole rectration, as sketched in Figs. 6.1 and 6.2. The local sheet thickness is defined by the function $h=h(x, t)$ for the planar and $h=h(r, t)$ for the axisymmetric problem.

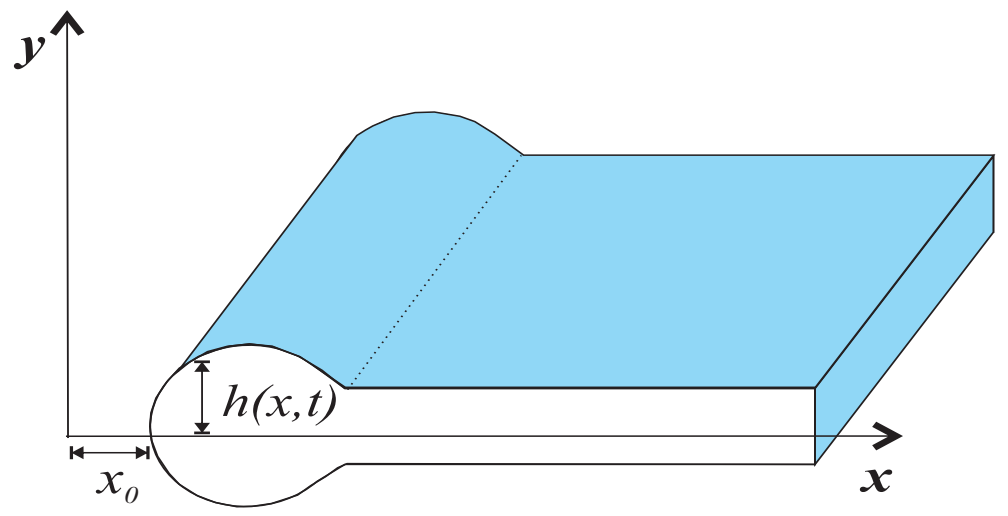

Figure 6.1: Sketch of flow domain for a straight line retraction in a planar liquid sheet.

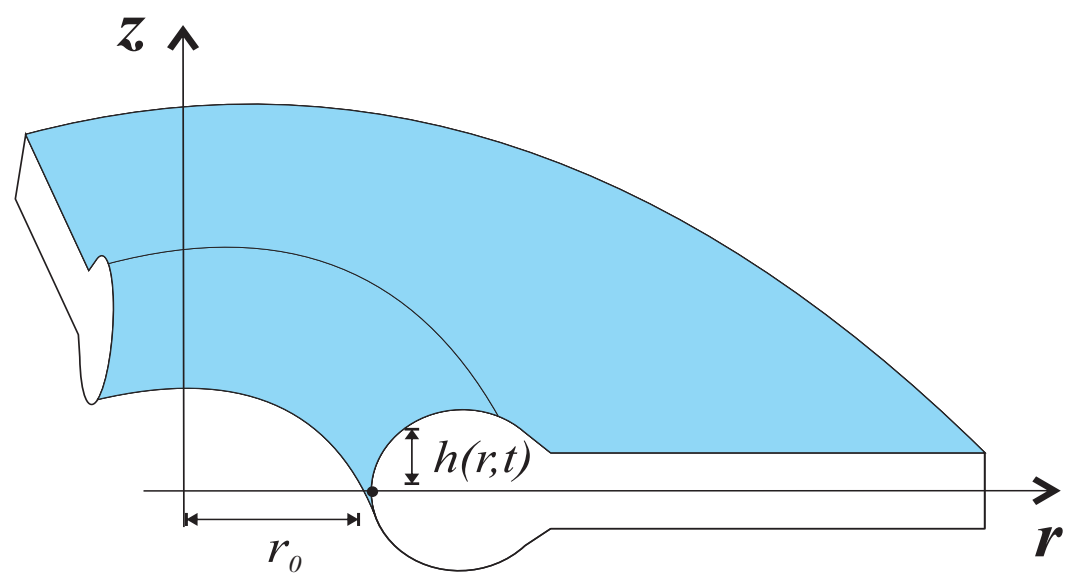

Figure 6.2: Sketch of flow domain for hole retraction in a planar liquid sheet. 
Boundary conditions along the liquid-gas interface are defined at $z=h$ and correspond to force balance along the normal and tangential direction, and kinematic condition as sketched in Fig 6.3.

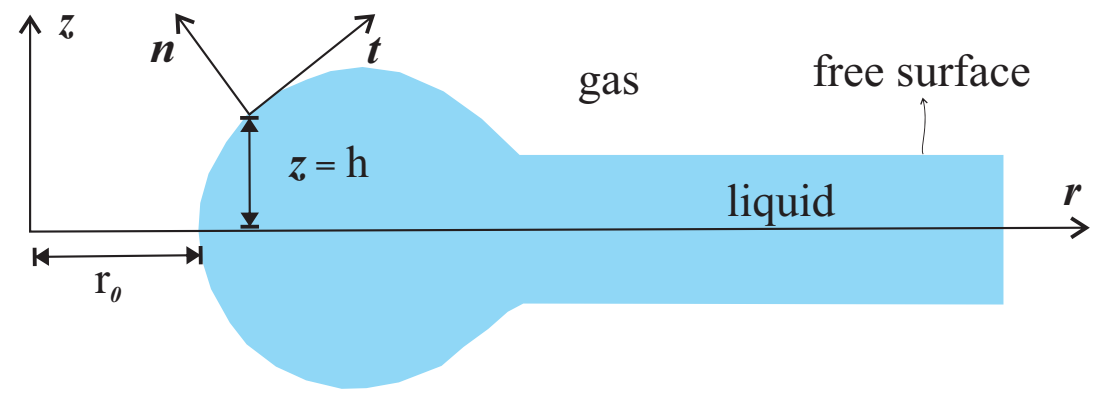

Figure 6.3: Sketch of orientation of normal unit vector $\mathbf{n}$ and tangential unit vector $\mathbf{t}$ for stress balance equation at interface of the fluid.

Considering that the gas viscosity is much smaller than the liquid viscosity, tangential stress at the interface is zero and normal stress is balanced with capillary force

$$
\begin{aligned}
& \text { n.T.n }=\sigma \nabla \cdot \mathbf{n} \\
& \text { n.T.t }=0 .
\end{aligned}
$$

as defined earlier in Chapter 2, the normal (n) and tangential (t) unit vectors are written as:

$$
\begin{aligned}
& \mathbf{t}=\left[\left(1+\left(\frac{\partial h}{\partial r}\right)^{2}\right)^{-1 / 2} \mathbf{e}_{r}, \quad \frac{\partial h}{\partial r}\left(1+\left(\frac{\partial h}{\partial r}\right)^{2}\right)^{-1 / 2} \mathbf{e}_{z}\right] \\
& \mathbf{n}=\left[-\frac{\partial h}{\partial r}\left(1+\left(\frac{\partial h}{\partial r}\right)^{2}\right)^{-1 / 2} \mathbf{e}_{r},\left(1+\left(\frac{\partial h}{\partial r}\right)^{2}\right)^{-1 / 2} \mathbf{e}_{z}\right]
\end{aligned}
$$

Kinematic condition imposes zero flow throught the liquid-gas interface, thus surface moves with the liquid velocity along at the boundary

$$
\frac{D h}{D t}=\mathbf{j} \cdot \mathbf{u}
$$

$\mathbf{j}$ is the unit vector in $z$ direction. 


\section{3}

\section{Initial condition}

Unsteady problems require imposed initial conditions to be solved. Here, the liquid film is considered stationary, and so the initial condition for the velocity field is set to be zero. The initial thickness profile that a fluid film assumes just after the pinch-off could be setted up based on the final solution obtained at Part I, however, in order to validate our numerical solution, initial profile was based on Savva and Bush [31] work; they proposed an initial profile that consists of a semicircular cap followed by a nearly straight line of constant thickness, as sketched in Fig. 6.4.

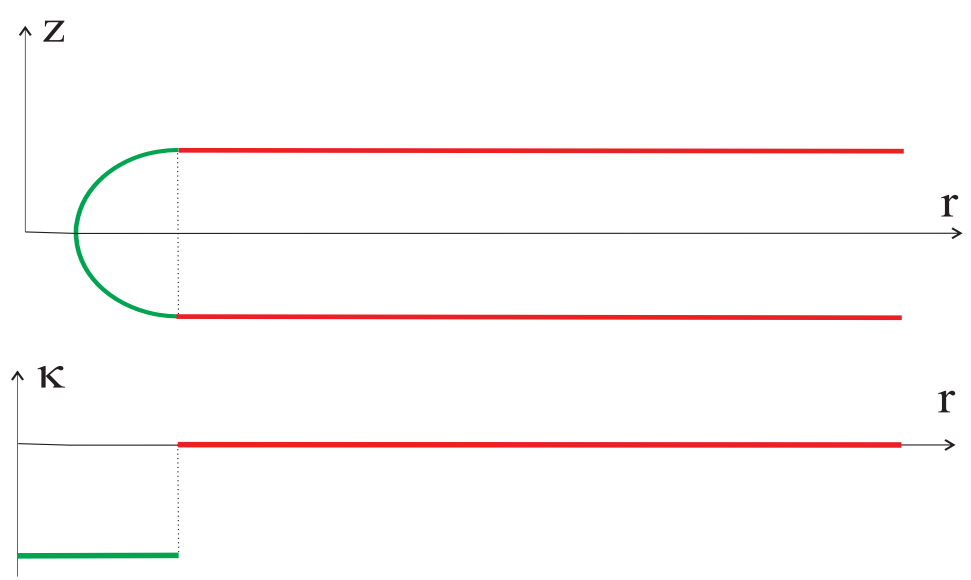

Figure 6.4: Sketch of the initial thickness profile, which is assumed to be semicircular cap followed by a straight line. The curvature has a initial negative value for the circular cap and then jumps to zero for the straight line.

Figure 6.4 also shows that the curvature has a negative constant value for the semicircular region, and then it jumps to zero when the straight line starts. Numerical implications of such discontinuity in the curvature, and the solution to remediate that are discussed in Section. 7.

\section{4}

\section{Lubrification approximation}

The large ratio of characteristic lengths along the directions parallel and perpendicular to the flow is also present in this problem. In this sense, the long wavelength approximation introduced in Section 2.3 is still valid here. Avoiding details that have already been explained in Section 2.3, the focus of this section is to derive the set of lubrication equation for straight line retraction, in $§ 6.4 .1$ and hole retraction in $\$ 6.4 .2$. 


\subsection{1}

\section{Straight line retraction}

Starting from Eqs. 6-1 and 6-2 in its two-dimensional Cartesian form:

$$
\begin{aligned}
& \frac{\partial U}{\partial x}+\frac{\partial V}{\partial y}=0 \\
& \rho\left(\frac{\partial U}{\partial t}+U \frac{\partial U}{\partial x}+V \frac{\partial U}{\partial y}\right)=-\frac{\partial P}{\partial x}+\frac{\partial T_{x x}}{\partial x}+\frac{\partial T_{x y}}{\partial y} \\
& \rho\left(\frac{\partial V}{\partial t}+U \frac{\partial V}{\partial x}+V \frac{\partial V}{\partial y}\right)=-\frac{\partial P}{\partial y}+\frac{\partial T_{x y}}{\partial x}+\frac{\partial T_{y y}}{\partial y}
\end{aligned}
$$

interface force balance conditions (Eqs. 6-7 and 6-8), and kinematic condition at the interface of the film (Eq. 6-10), also in two-dimensional Cartesian form

$$
\begin{aligned}
& {\left[\left(\frac{\partial h}{\partial x}\right)^{2}\left(T_{x x}-P\right)+\frac{\partial h}{\partial x} T_{y x}+T_{y y}-P\right]\left[1+\left(\frac{\partial h}{\partial x}\right)^{2}\right]^{-1}=\sigma \kappa} \\
& {\left[-\frac{\partial h}{\partial x} T_{x x}+\left[1-\left(\frac{\partial h}{\partial x}\right)^{2}\right] T_{y x}+\frac{\partial h}{\partial x} T_{y y}\right]\left[1+\left(\frac{\partial h}{\partial x}\right)^{2}\right]^{-1}=0} \\
& \frac{\partial h}{\partial t}+\left.U\right|_{y=h} \frac{\partial h}{\partial x}=\left.V\right|_{y=h},
\end{aligned}
$$

and assuming symmetry about $y=0$, one can perform the expansion presented in 2-19 around $y=0$ and introduce the expansions in the set of equations 6-11 to 6-13, 6-14 and 6-15. Afterwards, simillarly to section 2.3, one can assume the long wavelength approximation, and derive the lubrication system for a straight line film retraction of an Oldroyd-B fluid

$$
\begin{aligned}
& \frac{\partial h}{\partial t}+u \frac{\partial h}{\partial x}+h \frac{\partial u}{\partial x}=0 \\
& \frac{\partial u}{\partial t}+u \frac{\partial u}{\partial x}-\frac{\sigma}{\rho} \frac{\partial \kappa}{\partial x}-\frac{4 \eta_{s}}{\rho h} \frac{\partial h}{\partial x} \frac{\partial u}{\partial x}-\frac{4 \eta_{s}}{\rho} \frac{\partial^{2} u}{\partial x^{2}}-\frac{1}{\rho} \frac{\partial \tau_{x x}^{p}}{\partial x}, \\
& +\frac{1}{\rho} \frac{\partial \tau_{y y}^{p}}{\partial x}-\frac{\tau_{x x}^{p}}{\rho h} \frac{\partial h}{\partial x}+\frac{\tau_{y y}^{p}}{\rho h} \frac{\partial h}{\partial x}=0, \\
& \tau_{x x}^{p}+\lambda\left(\frac{\partial \tau_{x x}^{p}}{\partial t}+u \frac{\partial \tau_{x x}^{p}}{\partial x}-2 \tau_{x x}^{p} \frac{\partial u}{\partial x}\right)=2 \eta_{p} \frac{\partial u}{\partial x} \\
& \tau_{y y}^{p}+\lambda\left(\frac{\partial \tau_{y y}^{p}}{\partial t}+u \frac{\partial \tau_{y y}^{p}}{\partial x}+2 \tau_{y y}^{p} \frac{\partial u}{\partial x}\right)=-2 \eta_{p} \frac{\partial u}{\partial x}
\end{aligned}
$$


where $\kappa$ is the interface curvature given by

$$
\kappa(x, t)=\frac{1}{2}\left(\frac{\partial^{2} h}{\partial x^{2}}\right)\left[1+\frac{1}{4}\left(\frac{\partial h}{\partial x}\right)^{2}\right]^{-3 / 2} .
$$

Numerical simulation showed that the characteristic retraction time is prescribed by the length scale of the problem. For low viscosity regime, the motion is primarily concentrated near the edge. Conversely, for high viscosity regime, a larger portion of the film is accelerated by the unbalanced surface tension force close the tip. Thus, in order to obtain non-dimensional equations, Savva and Bush [31] proposed to use two different set of dimensionless parameters, depending on the relative importance of viscosity to surface tension, which is quantified through the Ohnersorge number $O h=\eta_{s} / \sqrt{2 H \rho \sigma}$. For a high viscosity or high $O h$ regime, the dimensionaless variables are defined as

$$
\begin{aligned}
t=\tau_{v i s c} t^{*}, \quad x=O h H x^{*}, & h=H h^{*}, \quad u=U_{c} u^{*}, \\
\tau_{x x}=\frac{\eta_{s} U_{c} O h}{H} \tau_{x x}^{*}, & \tau_{y y}=\frac{\eta_{s} U_{c}}{O h H} \tau_{y y}^{*},
\end{aligned}
$$

where $\tau_{\text {visc }}=O h H / U_{c}$ is the characteristic time scale for high $O h$, and $U_{c}=\sqrt{2 \sigma /(\rho H)}$ is the Taylor-Cullick velocity. For low viscosity regime, the dimensionaless variables are defined as:

$$
\begin{aligned}
t=\tau_{\text {inv }} t^{*}, \quad x=H x^{*}, & h=H h^{*}, \quad u=U_{c} u^{*}, \\
\tau_{x x}=\frac{\eta_{s} U_{c}}{H} \tau_{x x}^{*}, & \tau_{y y}=\frac{\eta_{s} U_{c}}{H} \tau_{y y}^{*},
\end{aligned}
$$

where $\tau_{\text {inv }}=H / U_{c}$ is the chacracteristic time scale for low $O h$.

The governing equations (6-17 to 6-20) can be rewritten in terms of the dimensionaless variables defined in 6-22, leading to the set of dimensionaless 
lubrication equations for high $\mathrm{Oh}$

$$
\begin{aligned}
\frac{\partial h}{\partial t}+u \frac{\partial h}{\partial x}+h \frac{\partial u}{\partial x}=0 & \\
\frac{\partial u}{\partial t}+u \frac{\partial u}{\partial x}-\frac{1}{2 O h^{2}} \frac{\partial \kappa}{\partial x}-\frac{4}{h} \frac{\partial}{\partial x}\left(h \frac{\partial u}{\partial x}\right) & -\frac{O h^{2}}{h} \frac{\partial}{\partial x}\left(\tau_{x x}^{p} h\right) \\
& +\frac{1}{h} \frac{\partial}{\partial x}\left(\tau_{y y}^{p} h\right)=0, \\
\tau_{x x}^{p}+D e\left(\frac{\partial \tau_{x x}^{p}}{\partial t}+u \frac{\partial \tau_{x x}^{p}}{\partial x}-2 \tau_{x x}^{p} \frac{\partial u}{\partial x}\right) & =-2 \frac{\eta_{r}}{O h^{2}} \frac{\partial u}{\partial x} \\
\tau_{y y}^{p}+D e\left(\frac{\partial \tau_{y y}^{p}}{\partial t}+u \frac{\partial \tau_{y y}^{p}}{\partial x}+2 \tau_{y y}^{p} \frac{\partial u}{\partial x}\right) & =2 \eta_{r} \frac{\partial u}{\partial x} .
\end{aligned}
$$

The dimensionaless interface curvature is given by:

$$
\kappa(x, t)=\frac{1}{2}\left(\frac{\partial^{2} h}{\partial x^{2}}\right)\left[1+\frac{1}{4} \frac{1}{O h^{2}}\left(\frac{\partial h}{\partial x}\right)^{2}\right]^{-3 / 2} .
$$

For low $O h$ regime, the dimensionaless set of equations becomes

$$
\begin{aligned}
& \frac{\partial h}{\partial t}+u \frac{\partial h}{\partial x}+h \frac{\partial u}{\partial x}=0, \\
& \frac{\partial u}{\partial t}+u \frac{\partial u}{\partial x}-\frac{1}{2} \frac{\partial \kappa}{\partial x}-\frac{4 O h}{h} \frac{\partial}{\partial x}\left(h \frac{\partial u}{\partial x}\right)-\frac{O h}{h} \frac{\partial}{\partial x}\left(\tau_{x x}^{p} h\right), \\
&+\frac{O h}{h} \frac{\partial}{\partial x}\left(\tau_{y y}^{p} h\right)=0,
\end{aligned}
$$

$$
\begin{aligned}
\tau_{x x}^{p}+D e\left(\frac{\partial \tau_{x x}^{p}}{\partial t}+u \frac{\partial \tau_{x x}^{p}}{\partial x}-2 \tau_{x x}^{p} \frac{\partial u}{\partial x}\right) & =2 \eta_{r} \frac{\partial u}{\partial x} \\
\tau_{y y}^{p}+D e\left(\frac{\partial \tau_{y y}^{p}}{\partial t}+u \frac{\partial \tau_{y y}^{p}}{\partial x}+2 \tau_{y y}^{p} \frac{\partial u}{\partial x}\right) & =-2 \eta_{r} \frac{\partial u}{\partial x} .
\end{aligned}
$$

The dimensionaless interface curvatures is now defined as:

$$
\kappa(x, t)=\frac{1}{2}\left(\frac{\partial^{2} h}{\partial x^{2}}\right)\left[1+\frac{1}{4}\left(\frac{\partial h}{\partial x}\right)^{2}\right]^{-3 / 2} .
$$




\subsection{2}

\section{Hole retraction}

For hole circular retraction flow, Eqs. 6-1 and 6-2 are written in the two-dimensional form for cylindrical coordinates:

$$
\begin{aligned}
& \frac{\partial}{\partial r}(U r)+\frac{\partial}{\partial z}(V r)=0 \\
& \rho\left(\frac{\partial U}{\partial t}+U \frac{\partial U}{\partial r}+V \frac{\partial U}{\partial z}\right)=-\frac{\partial P}{\partial r}+\frac{1}{r} \frac{\partial}{\partial r}\left(r T_{r r}\right)+\frac{\partial}{\partial z} T_{z r}-\frac{T_{\theta \theta}}{r}(6-3) \\
& \rho\left(\frac{\partial V}{\partial t}+U \frac{\partial V}{\partial r}+V \frac{\partial V}{\partial z}\right)=-\frac{\partial P}{\partial z}+\frac{1}{r} \frac{\partial}{\partial r}\left(r T_{z r}\right)+\frac{\partial}{\partial z} T_{z z}
\end{aligned}
$$

Force balance along the free surface and kinematic condition at the film interface are written in cylindrical coordinates as:

$$
\begin{aligned}
& {\left[\left(\frac{\partial h}{\partial r}\right)^{2}\left(T_{r r}-P\right)+\frac{\partial h}{\partial r} T_{z r}+T_{z z}-P\right]\left[1+\left(\frac{\partial h}{\partial r}\right)^{2}\right]^{-1}=\sigma \kappa(} \\
& {\left[\frac{\partial h}{\partial r} T_{r r}+\left[1-\left(\frac{\partial h}{\partial r}\right)^{2}\right] T_{r z}-\frac{\partial h}{\partial r} T_{z z}\right]\left[1+\left(\frac{\partial h}{\partial r}\right)^{2}\right]^{-1}=0} \\
& \frac{\partial h}{\partial t}+\left.U\right|_{z=h} \frac{\partial h}{\partial r}=V_{z=h}
\end{aligned}
$$

Following the same approach of subsection 6.4.1, given that the expansion of $T_{r r}$ and $T_{\theta \theta}$ are equal to the expansion for $T_{x x}$ and the expansion of $T_{r z}$ is equal to $T_{x y}$, one can obtain the lubrication system for a circular hole retraction of an Oldroyd-B liquid film:

$$
\begin{gathered}
\frac{\partial h}{\partial t}+\frac{1}{r} \frac{\partial}{\partial r}(h r u)=0 \\
\frac{\partial u}{\partial t}+u \frac{\partial u}{\partial r}-\frac{\sigma}{\rho} \frac{\partial \kappa}{\partial r}-\frac{4 \eta_{s}}{\rho h}\left[\frac{\partial}{\partial r}\left(\frac{h}{r} \frac{\partial}{\partial r}(u r)\right)-\frac{u}{2 r} \frac{\partial h}{\partial r}\right] \\
\quad+\frac{1}{\rho r} \frac{\partial}{\partial r}\left(h \tau_{z z}^{p}\right)-\frac{1}{\rho h r} \frac{\partial}{\partial r}\left(h r \tau_{r r}^{p}\right)+\frac{\tau_{\theta \theta}^{p}}{\rho r}=0, \\
\tau_{r r}^{p}+\lambda\left(\frac{\partial \tau_{r r}^{p}}{\partial t}+u \frac{\partial \tau_{r r}^{p}}{\partial r}-2 \tau_{r r}^{p} \frac{\partial u}{\partial r}\right)=2 \eta_{p} \frac{\partial u}{\partial r} \\
\tau_{z z}^{p}+\lambda\left(\frac{\partial \tau_{z z}^{p}}{\partial t}+u \frac{\partial \tau_{z z}^{p}}{\partial r}+\frac{\tau_{z z}^{p}}{r} \frac{\partial}{\partial r}(r u)\right)=-\frac{2 \eta_{p}}{r} \frac{\partial}{\partial r}(r u), \\
\tau_{\theta \theta}^{p}+\lambda\left(\frac{\partial \tau_{\theta \theta}^{p}}{\partial t}+u \frac{\partial \tau_{\theta \theta}^{p}}{\partial r}-2 \tau_{\theta \theta}^{p} \frac{u}{r}\right)=2 \eta_{p} \frac{u}{r} .
\end{gathered}
$$


The interface curvature is defined as:

$$
\kappa=\frac{1}{2} \frac{\partial^{2} h}{\partial r^{2}}\left[1+\frac{1}{4}\left(\frac{\partial h}{\partial r}\right)^{2}\right]^{-3 / 2}-\frac{1}{2} \frac{1}{r} \frac{\partial h}{\partial r}\left[1+\frac{1}{4}\left(\frac{\partial h}{\partial r}\right)^{2}\right]^{-1 / 2} .
$$

Dimensionaless variable are defined as the straight line retraction problem. For high $O h$ regime are defined as:

$$
\begin{aligned}
& t=\tau_{v i s c} t^{*}, \quad r=O h H r^{*}, \quad h=H h^{*}, \quad u=U_{c} u^{*}, \\
& \tau_{r r}=\frac{\eta_{s} U_{c} O h}{H} \tau_{r r}^{*}, \quad \tau_{y y}=\frac{\eta_{s} U_{c}}{O h H} \tau_{y y}^{*}, \quad \tau_{\theta \theta}=\frac{\eta_{s} U_{c}}{H} \tau_{\theta \theta}^{*},
\end{aligned}
$$

and for low $O h$ regime, defined as

$$
\begin{aligned}
& t=\tau_{i n v} t^{*}, \quad r=H r^{*}, \quad h=H h^{*}, \quad u=U_{c} u^{*}, \\
& \tau_{r r}=\frac{\eta_{s} U_{c}}{H} \tau_{r r}^{*}, \quad \tau_{y y}=\frac{\eta_{s} U_{c}}{H} \tau_{y y}^{*}, \quad \tau_{\theta \theta}=\frac{\eta_{s} U_{c}}{H} \tau_{\theta \theta}^{*}, .
\end{aligned}
$$

In this sense, the dimensionaless form of Eqs. 6-40 to 6-44 for high Oh regime is:

$$
\begin{gathered}
\frac{\partial h}{\partial t}+\frac{1}{r} \frac{\partial}{\partial r}(h r u)=0 \\
\frac{\partial u}{\partial t}+u \frac{\partial u}{\partial r}-\frac{1}{2 O h^{2}} \frac{\partial \kappa}{\partial r}-\frac{4}{h}\left[\frac{\partial}{\partial r}\left(\frac{h}{r} \frac{\partial}{\partial r}(u r)\right)-\frac{u}{2 r} \frac{\partial h}{\partial r}\right] \\
+\frac{1}{h} \frac{\partial}{\partial r}\left(h \tau_{z z}^{p}\right)-\frac{O h^{2}}{h r} \frac{\partial}{\partial r}\left(h r \tau_{r r}^{p}\right)+O h \frac{\tau_{\theta \theta}^{p}}{r}=0 \\
\tau_{r r}^{p}+D e\left(\frac{\partial \tau_{r r}^{p}}{\partial t}+u \frac{\partial \tau_{r r}^{p}}{\partial r}-2 \tau_{r r}^{p} \frac{\partial u}{\partial r}\right)=2 \frac{\eta_{r}}{O h^{2}} \frac{\partial u}{\partial r} \\
\tau_{z z}^{p}+D e\left(\frac{\partial \tau_{z z}^{p}}{\partial t}+u \frac{\partial \tau_{z z}^{p}}{\partial r}+\frac{\tau_{z z}^{p}}{r} \frac{\partial}{\partial r}(r u)\right)=-\frac{2 \eta_{r}}{r} \frac{\partial}{\partial r}(r u) \\
\tau_{\theta \theta}^{p}+D e\left(\frac{\partial \tau_{\theta \theta}^{p}}{\partial t}+u \frac{\partial \tau_{\theta \theta}^{p}}{\partial r}-2 \tau_{\theta \theta}^{p} \frac{u}{r}\right)=2 \frac{\eta_{r}}{O h} \frac{u}{r}
\end{gathered}
$$


Conversely, the for low $O h$ regime, it is written as:

$$
\begin{aligned}
& \frac{\partial h}{\partial t}+ \frac{1}{r} \frac{\partial}{\partial r}(h r u)=0, \\
& \frac{\partial u}{\partial t}+u \frac{\partial u}{\partial r}-\frac{1}{2} \frac{\partial \kappa}{\partial r}-\frac{4 O h}{h}\left[\frac{\partial}{\partial r}\left(\frac{h}{r} \frac{\partial}{\partial r}(u r)\right)-\frac{u}{2 r} \frac{\partial h}{\partial r}\right] \\
&+\frac{O h}{r} \frac{\partial}{\partial r}\left(h \tau_{z z}^{p}\right)-\frac{O h}{h r} \frac{\partial}{\partial r}\left(h r \tau_{r r}^{p}\right)+O h \frac{\tau_{\theta \theta}^{p}}{r}=0, \\
& \tau_{r r}^{p}+D e\left(\frac{\partial \tau_{r r}^{p}}{\partial t}+u \frac{\partial \tau_{r r}^{p}}{\partial r}-2 \tau_{r r}^{p} \frac{\partial u}{\partial r}\right)=-2 \eta_{r} \frac{\partial u}{\partial r}, \\
& \tau_{z z}^{p}+D e\left(\frac{\partial \tau_{z z}^{p}}{\partial t}+u \frac{\partial \tau_{z z}^{p}}{\partial r}+\frac{\tau_{z z}^{p}}{r} \frac{\partial}{\partial r}(r u)\right)=-\frac{2 \eta_{r}}{r} \frac{\partial}{\partial r}(r u), \\
& \tau_{\theta \theta}^{p}+D e\left(\frac{\partial \tau_{\theta \theta}^{p}}{\partial t}+u \frac{\partial \tau_{\theta \theta}^{p}}{\partial r}-2 \tau_{\theta \theta}^{p} \frac{u}{r}\right)=2 \eta_{r} \frac{u}{r} .
\end{aligned}
$$

It is possible to observe that the set of lubrication equations that describes a film retraction is similar to the one used to describe the film rupture flow, with the absence of the van der Waals term. However, the retraction flow is free boundary problem; the positions of the tip of the rim, and consequently the flow domain, changes with time. This characteristic leads to a more complicated solution method, which is described in Section 7. 


\section{Solution method}

This chapter brings the numerical methodology used to solve the full set of coupled non-linear equations proposed to describe sheet retraction process. Similar to the rupture process, a second-order finite difference method was used to discretize the spatial derivatives, and an implicit Crank-Nicholson method to discretize the time derivative terms. However, the film retraction brings extra difficulties; the tip or edge velocity, which is one of boundary conditions is also part of problem solution, also the thickness derivative goes to infinity in the tip, and the initial condition in the form that was set in section 6.3 has a jump in curvature, creating a point of sigularity.

There is no single way to deal with the difficulties that arise during the numerical solution of sheet retraction. For this work, the chosen approach is based on the Savva and Bush's work [31] and presented as follows: Section 7.1 presents the methodology used to solve the tip velocity. Section 7.3 describes mapping domain transformation performed to avoid the blowing up of the thickness derivative at the tip, and Section 7.4 brings the strategy to avoid the discontinuity in the curvature for the initial thickness profile.

\section{1 \\ Velocity tip condition}

The long wavelength approximation used to derive Eqs 6-24 to 6-27 and 6-40 to 6-44 fails as equations approach to the edge of the liquid sheet, since $\partial h / \partial x \rightarrow \infty$ in this region. Eggers and Dupont [42] faced the same problem in studying jet breakup, they overcame this difficulty by neglecting the viscosity effect near to the tip, which for a jet flow was not critical. However for the film retraction problem, viscosity plays an important role by hindering the expansion rate of a straight line or a hole, and neglecting its effect leads to a totally inaccurate problem solution. Knowing it, Savva and Bush [31] proposed a more appropriate approach to solve the tip velocity: instead of use the long wavelength approximation, they used the condition $\partial h / \partial x \rightarrow \infty$ to simplified boundary conditions at the tip. The approach is detailed below, for a straight line 7.1.1 and a hole 7.1.2. 


\subsection{1}

\section{Straight line tip velocity}

For the tip velocity of a straight line retraction, the same procedure adopted in Section 2.3 is followed; Taylor expansions given in 2-19 are used in Eqs. 6-1 and 6-2 to derive the set of expanded conservation equations

$$
\begin{aligned}
& v_{1}=-\frac{\partial u}{\partial x} \\
& \rho\left(\frac{\partial u}{\partial t}+u \frac{\partial u}{\partial x}\right)=-\frac{\partial p}{\partial x}+\frac{\partial \tau_{x x}}{\partial x}+\tau_{y x} .
\end{aligned}
$$

and in Eqs. 6-7 and 6-8 to get the expanded boundary conditions

$$
\begin{aligned}
& {\left[\left(\frac{\partial h}{\partial x}\right)^{2}\left(\tau_{x x}-p\right)+\frac{\partial h}{\partial r} \tau_{y x} h+\tau_{y y}-p\right]\left[1+\left(\frac{\partial h}{\partial r}\right)^{2}\right]^{-1}=\sigma \kappa} \\
& {\left[-\frac{\partial h}{\partial x} \tau_{x x}+\left[1-\left(\frac{\partial h}{\partial x}\right)^{2}\right] \tau_{y x} h+\frac{\partial h}{\partial x} \tau_{y y}\right]\left[1+\left(\frac{\partial h}{\partial x}\right)^{2}\right]^{-1}=0}
\end{aligned}
$$

Knowing that $\partial h / \partial x \rightarrow \infty$ in the vicinity of the edge of the film, it is reasonable to make the following approximation

$$
1+\left(\frac{\partial h}{\partial r}\right)^{2} \approx\left(\frac{\partial h}{\partial r}\right)^{2}
$$

The approximation 7-5 can be used into Eqs. 7-3 and 7-4 and get a simplified form for tip boundary conditions

$$
\begin{aligned}
& -p+\tau_{x x}+2\left(\frac{\partial h}{\partial x}\right)^{-1} \tau_{y x} h+\left(\frac{\partial h}{\partial x}\right)^{-2} \tau_{y y}=\sigma \kappa \\
& -\tau_{x x}-\left(\frac{\partial h}{\partial x}\right) \tau_{y x} h+\tau_{y y}=0 .
\end{aligned}
$$

Given that $(\partial h / \partial x)^{-1} \rightarrow 0$, one can get an expression for mechanical pressure at the tip in function of stress tensor components from Eq.7-6 , where $\tau_{x x}=\tau_{x x}^{p}+2 \eta_{s}(\partial u / \partial x)$

$$
-p=-\tau_{x x}^{p}-2 \eta_{s} \frac{\partial u}{\partial x}+\sigma \kappa
$$

Substituing 7-8 into expanded Cauchy equation 7-2, on can get the expression for the retraction velocity of the tip $u_{0}$ of a straight line in Oldroyd- 
B fluid

$$
\frac{\partial u_{0}}{\partial t}+u_{0} \frac{\partial u_{0}}{\partial x}=\frac{\sigma}{\rho} \frac{\partial \kappa}{\partial x}-\frac{\eta_{s}}{\rho} \frac{\partial^{2} u_{0}}{\partial x^{2}}+\frac{\tau_{y x, 0}}{\rho}
$$

where $\tau_{x y, 0}$ is given by Eq. 7-7, being $\tau_{x x}=\tau_{x x}^{p}+2 \eta_{s}(\partial u / \partial x)$ and $\tau_{y y}=$ $\tau_{y y}^{p}+2 \eta_{s}(\partial u / \partial x)$

$$
\tau_{y x_{0}}=-\left(h \frac{\partial h}{\partial x}\right)^{-1}\left(\tau_{x x}^{p}-\tau_{y y}^{p}+4 \eta_{s} \frac{\partial u}{\partial x}\right)
$$

Here, unlike Savva and Bush [31], the term $\tau_{x y}$ (in their case $u_{2}$ ) was not found by extrapolation, but by applying the approximation in the expanded tangential boundary condition.

The non-dimensional form for a high $O h$ regime can found by using the non-dimensional parameters presented at 6-22

$$
\frac{\partial u_{0}}{\partial t}+u_{0} \frac{\partial u_{0}}{\partial x}-\frac{1}{2 O h^{2}} \frac{\partial \kappa}{\partial x}+\frac{\partial^{2} u_{0}}{\partial x^{2}}-\tau_{y x, 0}=0
$$

Conversely, the non-dimensional form of the tip velocity for low $O h$ is given by

$$
\frac{\partial u_{0}}{\partial t}+u_{0} \frac{\partial u_{0}}{\partial x}-\frac{1}{2} \frac{\partial \kappa}{\partial x}+O h \frac{\partial^{2} u_{0}}{\partial x^{2}}-O h \tau_{y x, 0}=0
$$

\subsection{2}

\section{Hole tip velocity}

The tip velocity of a hole retraction follows the same procedure presented in above section, but for equations written in cylindrical coordinates. Here, intermediate steps are skipped to avoid unnecessary repetitiveness. In this sense, using approximations $(\partial h / \partial r) \rightarrow \infty$ and $(\partial h / \partial r)^{-1} \rightarrow 0$, the equation of the tip velocity of the hole retraction in an Oldroyd-B fluid is given by

$\frac{\partial u_{0}}{\partial t}+u_{0} \frac{\partial u_{0}}{\partial r}-\frac{\sigma}{\rho} \frac{\partial \kappa}{\partial r}-\frac{\tau_{r r, 0}^{p}}{\rho r}+\frac{\tau_{r z, 0}}{\rho}+\frac{\tau_{\theta \theta, 0}}{\rho r}+\frac{\eta_{s}}{\rho}\left(\frac{\partial^{2} u_{0}}{\partial r^{2}}-\frac{1}{r} \frac{\partial u_{0}}{\partial r}+\frac{u_{0}}{r^{2}}\right)=0$

and $\tau_{r z, 0}$ corresponds to

$$
\tau_{r z, 0}=-\frac{2}{h}\left(\frac{\partial h}{\partial r}\right)^{-1}\left[\tau_{r r}^{p}-\tau_{z z}^{p}\right]
$$


The non-dimensional form of Eq.7-11 for high Oh regime is given by

$\frac{\partial u_{0}}{\partial t}+u_{0} \frac{\partial u_{0}}{\partial r}-\frac{1}{2 O h^{2}} \frac{\partial \kappa}{\partial r}-O h^{2} \frac{\tau_{r r, 0}^{p}}{r}+\tau_{r z, 0}+O h \frac{\tau_{\theta \theta, 0}}{r}+\left(\frac{\partial^{2} u_{0}}{r^{2}}-\frac{1}{r} \frac{\partial u_{0}}{\partial r}+\frac{u_{0}}{r^{2}}\right)=0$

and for low $O h$ the tip velocity equation becomes

$\frac{\partial u_{0}}{\partial t}+u_{0} \frac{\partial u_{0}}{\partial r}-\frac{1}{2} \frac{\partial \kappa}{\partial r}-O h \frac{\tau_{r r, 0}^{p}}{r}+O h \tau_{r z, 0}+O h \frac{\tau_{\theta \theta, 0}}{r}+O h\left(\frac{\partial^{2} u_{0}}{r^{2}}-\frac{1}{r} \frac{\partial u_{0}}{\partial r}+\frac{u_{0}}{r^{2}}\right)=0$

\section{2}

\section{Curvature singularity problem}

As discussed earlier, at the tip of the fluid film $\partial h / \partial r \rightarrow \infty$ creating numerical difficulties to evaluate the curvature at this point. Eggers and Duppont [42] overcame this problem by using an even quadratic polynomial to fit the jet profile in this region. A more elegant solution was proposed by Savva and Bush [31]; instead of creating a fitting, they rearranged equations using $f=h^{2}$. Hence, the equations are transformed accordingly, Eqs. 6-24 to 6-27 for the straight line retraction become

$$
\begin{aligned}
& \frac{\partial f}{\partial t}+u \frac{\partial f}{\partial x}+2 f \frac{\partial u}{\partial x}=0 \\
& \frac{\partial u}{\partial t}+u \frac{\partial u}{\partial x}-\frac{1}{2 O h^{2}} \frac{\partial \kappa}{\partial x}-\frac{2}{f} \frac{\partial f}{\partial x} \frac{\partial u}{\partial x}-4 \frac{\partial^{2} u}{\partial x^{2}}-\frac{O h^{2} \tau_{x x}^{p}}{2 f} \frac{\partial f}{\partial x}-O h^{2} \frac{\partial \tau_{x x}^{p}}{\partial x} \\
& +\frac{\tau_{y y}^{p}}{2 f} \frac{\partial f}{\partial x}+\frac{\partial \tau_{y y}^{p}}{\partial x}=0 \\
& \tau_{x x}^{p}+D e\left(\frac{\partial \tau_{x x}^{p}}{\partial t}+u \frac{\partial \tau_{x x}^{p}}{\partial x}-2 \tau_{x x}^{p} \frac{\partial u}{\partial x}\right)=-2 \frac{\eta_{r}}{O h^{2}} \frac{\partial u}{\partial x} \\
& \tau_{y y}^{p}+D e\left(\frac{\partial \tau_{y y}^{p}}{\partial t}+u \frac{\partial \tau_{y y}^{p}}{\partial x}+2 \tau_{y y}^{p} \frac{\partial u}{\partial x}\right)=2 \eta_{r} \frac{\partial u}{\partial x} .
\end{aligned}
$$

and the transformed curvature for straight line is given by

$$
\kappa=8 \frac{2 f \frac{\partial^{2} f}{\partial r^{2}}-\left(\frac{\partial f}{\partial r}\right)^{2}}{\left(16 f+\left(\frac{\partial f}{\partial r}\right)^{2}\right)^{3 / 2}}
$$


For hole retraction the transformation leads Eqs. 6-47 to 6-51 to

$$
\begin{aligned}
& \frac{\partial f}{\partial t}+u \frac{\partial f}{\partial r}+2 f \frac{\partial u}{\partial r}+2 \frac{f u}{r}=0 \\
& \frac{\partial u}{\partial t}+u \frac{\partial u}{\partial r}-\frac{1}{2 O h^{2}} \frac{\partial \kappa}{\partial r}-4 \frac{\partial^{2} u}{\partial r^{2}}-\frac{4}{r} \frac{\partial u}{\partial r}+4 \frac{u}{r^{2}}-\frac{2}{f} \frac{\partial f}{\partial r} \frac{\partial u}{\partial r}-\frac{1}{f} \frac{\partial f}{\partial r} \frac{u}{r} \\
& +\frac{\partial \tau_{z z}^{p}}{\partial r}+\frac{\tau_{z z}^{p}}{2 f} \frac{\partial f}{\partial r}-O h^{2} \frac{\partial \tau_{r r}^{p}}{\partial r}-\frac{O h^{2} \tau_{r r}^{p}}{r}-\frac{O h^{2}}{2} \frac{\tau_{r r}}{f} \frac{\partial f}{\partial r}+O h \frac{\tau_{\theta \theta}}{r}=(7-23) \\
& \tau_{r r}^{p}+D e\left(\frac{\partial \tau_{r r}^{p}}{\partial t}+u \frac{\partial \tau_{r r}^{p}}{\partial r}-2 \tau_{r r}^{p} \frac{\partial u}{\partial r}\right)=-2 \frac{\eta_{r}}{O h^{2}} \frac{\partial u}{\partial r} \\
& \tau_{z z}^{p}+D e\left(\frac{\partial \tau_{z z}^{p}}{\partial t}+u \frac{\partial \tau_{z z}^{p}}{\partial r}+\frac{\tau_{z z}^{p}}{r} \frac{\partial}{\partial r}(r u)\right)=-\frac{2 \eta_{r}}{r} \frac{\partial}{\partial r}(r u) \\
& \tau_{\theta \theta}^{p}+D e\left(\frac{\partial \tau_{\theta \theta}^{p}}{\partial t}+u \frac{\partial \tau_{\theta \theta}^{p}}{\partial r}-2 \tau_{\theta \theta}^{p} \frac{u}{r}\right)=2 \frac{\eta_{r}}{O h} \frac{u}{r} .
\end{aligned}
$$

and curvature is written as

$$
\kappa=8 \frac{2 f \frac{\partial^{2} f}{\partial r^{2}}-\left(\frac{\partial f}{\partial r}\right)^{2}}{\left(16 f+\left(\frac{\partial f}{\partial r}\right)^{2}\right)^{3 / 2}}-\frac{\frac{\partial f}{\partial r}}{r\left(16 f+\left(\frac{\partial f}{\partial r}\right)^{2}\right)^{1 / 2}}
$$

\section{3}

\section{Mapping domain transformation}

As the problem is free boundary, the solution domain changes with time due to the movement of the edge of the liquid sheet, requiring a re-meshing of the domain at each time step to accommodate that change. This re-meshing makes the numerical solution further complicated, thus to avoid it Savva and Bush [31] proposed the use of a transformation from physical $(r, t)$ to a computational domain $\left(r^{\prime}, t^{\prime}\right)$. The transformation is given by the following relation

$$
\begin{aligned}
& r=\left(1-\frac{x_{0}(t)}{L}\right) x^{\prime}+x_{0}(t) \\
& t=t^{\prime}
\end{aligned}
$$

for straight line retraction, where $0 \leq x^{\prime} \leq L$, being $\mathrm{L}$ the longitudinal extent of the liquid sheet. Under this mapping, the tip of the film is always located at $x^{\prime}=0$ and the free-boundary problem is transformed into a fixed-boundary 
problem. The derivatives are transformed accordingly

$$
\begin{aligned}
& \frac{\partial *}{\partial t}=\frac{\partial}{\partial t^{\prime}}-\left[\frac{1-\frac{x^{\prime}}{L}}{1-\frac{x_{0}(t)}{L}}\right] u_{0} \frac{\partial *}{\partial x^{\prime}} \\
& \frac{\partial *}{\partial x}=\frac{1}{1-\frac{x_{0}(t)}{L}} \frac{\partial}{\partial x^{\prime}}
\end{aligned}
$$

where the tip speed is given by the derivative of the tip position with time

$$
u_{0}(t)=\frac{\partial x_{0}(t)}{\partial t}
$$

Defining

$$
c=\frac{1}{1-\frac{x_{0}(t)}{L}}
$$

and dropping primes, Eqs. 7-17 to 7-20 can be written in computational domain as following

$$
\begin{gathered}
\frac{\partial f}{\partial t}+c\left[u-\left(1-\frac{x}{L}\right) u_{0}\right] \frac{\partial f}{\partial x}+2 c f \frac{\partial u}{\partial x}=0 \\
\frac{\partial u}{\partial t}+c\left[u-\left(1-\frac{x}{L}\right) u_{0}\right] \frac{\partial u}{\partial x}-\frac{c}{2 O h^{2}} \frac{\partial \kappa}{\partial x}-\frac{2 c^{2}}{f} \frac{\partial f}{\partial x} \frac{\partial u}{\partial x}-4 c^{2} \frac{\partial^{2} u}{\partial x^{2}} \\
-\frac{c O h^{2}}{2} \frac{\tau_{x x}^{p}}{f} \frac{\partial f}{\partial x}-c O h^{2} \frac{\partial \tau_{x x}^{p}}{\partial x}+\frac{c \tau_{y y}^{p}}{2 f} \frac{\partial f}{\partial x}+c \frac{\partial \tau_{y y}^{p}}{\partial x}=0 \\
\tau_{x x}^{p}+D e\left[\frac{\partial \tau_{x x}^{p}}{\partial t}+c\left(u-\left[1-\frac{x}{L}\right] u_{0}\right) \frac{\partial \tau_{x x}^{p}}{\partial x}-2 c \frac{\partial u}{\partial x} \tau_{x x}^{p}\right] \\
=-2 c \frac{\eta_{r}}{\partial h^{2}} \frac{\partial u}{\partial x} \\
\tau_{y y}^{p}+D e\left[\frac{\partial \tau_{y y}^{p}}{\partial t}+c\left(u-\left[1-\frac{x}{L}\right] u_{0}\right) \frac{\partial \tau_{y y}^{p}}{\partial x}+2 c \frac{\partial u}{\partial x} \tau_{y y}^{p}\right] \\
=2 c \eta_{r} \frac{\partial u}{\partial x}
\end{gathered}
$$

being the curvature transformed into

$$
\kappa=8 c^{2} \frac{2 f \frac{\partial^{2} f}{\partial x^{2}}-\left(\frac{\partial f}{\partial x}\right)^{2}}{\left(16 f+c^{2}\left(\frac{\partial f}{\partial x}\right)^{2}\right)^{3 / 2}}
$$


For hole retraction, $x$ is replaced by $r$, so the computacional domain is $0 \leq r^{\prime} \leq L, L$ becomes the radial extend of the liquid sheet, and tip is located at $r^{\prime}=0$. Thus, the tip speed is given by

$$
u_{0}(t)=\frac{\partial r_{0}(t)}{\partial t}
$$

And $c$ is written as

$$
c=\frac{1}{1-\frac{r_{0}(t)}{L}}
$$

Therefore, the transformation yields the system

$$
\begin{aligned}
& \frac{\partial f}{\partial t}+c\left[u-\left(1-\frac{r}{L}\right) u_{0}\right] \frac{\partial f}{\partial r}+2 c f \frac{\partial u}{\partial r}+2 c \frac{f u}{r+r_{0} c}=0 \\
& \frac{\partial u}{\partial t}+c\left[u-\left(1-\frac{r}{L}\right) u_{0}\right] \frac{\partial u}{\partial r}-\frac{c}{2 O h^{2}} \frac{\partial \kappa}{\partial r}-4 c^{2} \frac{\partial^{2} u}{\partial r^{2}}-\frac{4 c^{2}}{r+r_{0} c} \frac{\partial u}{\partial r} \\
& +4 c^{2} \frac{u}{\left(r+r_{0} c\right)^{2}}-\frac{2 c^{2}}{f} \frac{\partial f}{\partial r} \frac{\partial u}{\partial r}-\frac{c^{2}}{f} \frac{\partial f}{\partial r} \frac{u}{r+r_{0} c}+c \frac{\partial \tau_{z z}^{p}}{\partial r}+c \frac{\tau_{z z}^{p}}{2 f} \frac{\partial f}{\partial r} \\
& -c O h^{2} \frac{\partial \tau_{r r}^{p}}{\partial r}-c \frac{O h^{2} \tau_{r r}^{p}}{r+r_{0} c}-c \frac{O h^{2}}{2} \frac{\tau_{r r}}{f} \frac{\partial f}{\partial r}+c O h \frac{\tau_{\theta \theta}}{r+r_{0} c}=0 \\
& \tau_{r r}^{p}+D e\left(\frac{\partial \tau_{r r}^{p}}{\partial t}+c\left[u\left(1-\frac{r}{L}\right) u_{0}\right] \frac{\partial \tau_{r r}^{p}}{\partial r}-2 c \tau_{r r}^{p} \frac{\partial u}{\partial r}\right)=-2 c \frac{\eta_{r}}{O h^{2}} \frac{\partial u}{\partial r} \\
& \tau_{z z}^{p}+D e\left(\frac{\partial \tau_{z z}^{p}}{\partial t}+c\left[u\left(1-\frac{r}{L}\right) u_{0}\right] \frac{\partial \tau_{z z}^{p}}{\partial r}+c \frac{\tau_{z z}^{p} u}{r+r_{0} c}+c \tau_{z z}^{p} \frac{\partial u}{\partial r}\right) \\
& \tau_{\theta \theta}^{p}+D e\left(\frac{\partial \tau_{\theta \theta}^{p}}{\partial t}+c\left[u\left(1-\frac{r}{L}\right) u_{0}\right] \frac{\partial \tau_{\theta \theta}^{p}}{\partial r}-2 c \tau_{\theta \theta}^{p} \frac{u}{r+r_{0} c}\right)=2 c \frac{\eta_{r}}{O h} \frac{u}{r+r_{0} c}
\end{aligned}
$$

with curvature given by

$$
\kappa=c^{2}\left(8 \frac{2 f \frac{\partial^{2} f}{\partial r^{2}}-\left(\frac{\partial f}{\partial r}\right)^{2}}{\left(16 f+c^{2}\left(\frac{\partial f}{\partial r}\right)^{2}\right)^{3 / 2}}-\frac{\frac{\partial f}{\partial r}}{\left(r+r_{0} c\right)\left(16 f+c^{2}\left(\frac{\partial f}{\partial r}\right)^{2}\right)^{1 / 2}}\right)
$$

Equations 7-27, 7-29 to 7-32, as well as 7-34, 7-36 to 7-40 constitute hybrid Lagrangian-Eulerian systems; the computational domain for $x \neq 0$ or $r \neq 0$ is prescribed by an Eulerian formulation, and tip is treated as a Lagrangian point. 


\section{4}

\section{Initial thickness profile}

In section 6.3 the initial thickness profile is defined as semicircular cap couple with a nearly linear straight strip. This profile introduces a discontinuity in the curvature, which requires a much more refined mesh to computing the numerical solution of the problem, and consequently increases the computational time. To avoid it, Savva and Bush [31] adapted the initial condition to smoother profile by introducing a parameter $\alpha$ that controls the curvature,

$$
f(r, 0, \alpha)=1-\left(\frac{1}{2}-\alpha-r+\frac{1}{2} \sqrt{(1+2 \alpha)^{2}+4 r(2 \alpha+r-1)}\right)^{2} .
$$

The typically values for parameter used by Savva and Bush [31] were $\alpha=1 / 20$ to $\alpha=1 / 100$. In Fig.7.1 it is possible to observe how the profile changes as $\alpha$ is increased.

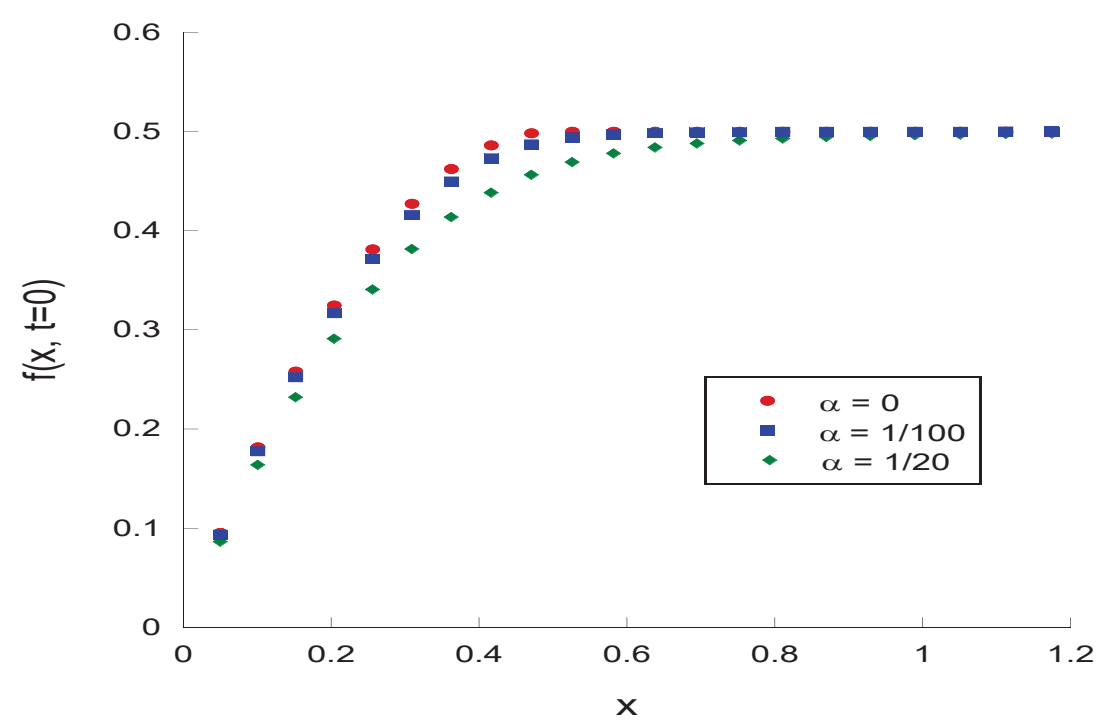

Figure 7.1: Initial thickness profile gets smoother as the value of $\alpha$ is augmented.

\section{5}

\section{Finite difference method}

Similarly to Section 4.1, a numerical approximation of the transport equation solutions was obtained by using the Finite Difference Method. Also, as can be observed in Fig.7.2, a staggered grid over the interval $0 \leq r \leq 1$ was used to avoid spurious oscillations in the curvature in the long-time limit; values 
of $h_{i}, \tau_{r r, i}, \tau_{z z, i}$ and $\tau_{\theta \theta, i}$ are prescribed at $r_{i}$ and values of $u_{i}$ are prescribes at $r_{i+1 / 2}$.

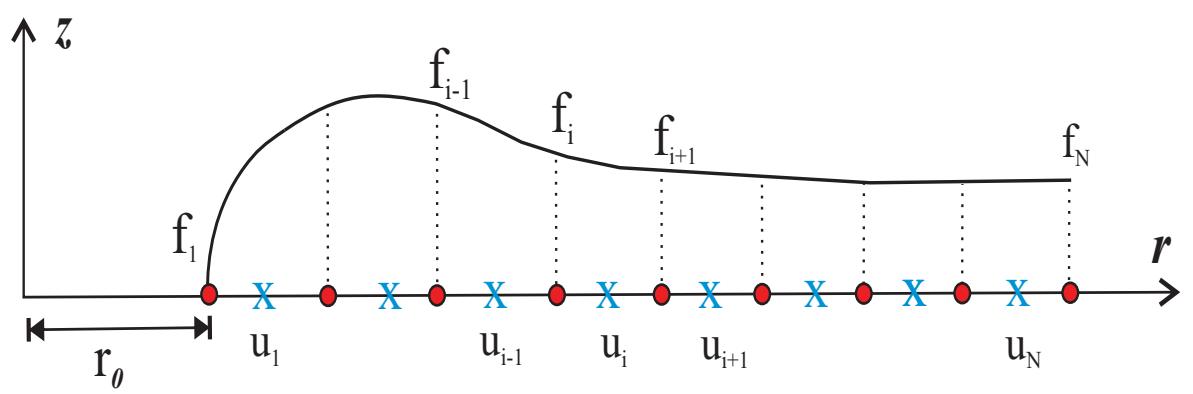

Figure 7.2: Stagerred grid used to solve retraction problem. Values of $f_{i}$ are prescribed at mesh points $r_{i}$, and values of $u_{i}$ are prescribed at $r_{i+1 / 2}=$ $\left(r_{i}+r_{i+1}\right) / 2$

Derivatives were performed by using second-order central-difference scheme, as explained in details at Section 4.1. And a non-uniform grid was also implemented to concentrate mesh near the tip of the film by using the function

$$
r_{i}=1-\left[\frac{N-i}{N-1}\right]^{a}
$$

For the numerical solution the initial value of the hole was set to be $r_{0}=50$ in all cases.

\section{6}

\section{Numerical validation}

In order to validate our numerical procedure, the solution of film retraction in a Newtonian fluid was compared with results presented by Savva and Bush [31]. Figures 7.3 and 7.4 show that the results of straight line and hole retraction for an $\mathrm{Oh}=10$ presents a good agreement with Savva and Bush results. A small numerical oscillation can be observed at the beginning of the retraction process, such oscillation can be associated with the high acceleration in the early stages of the process. 


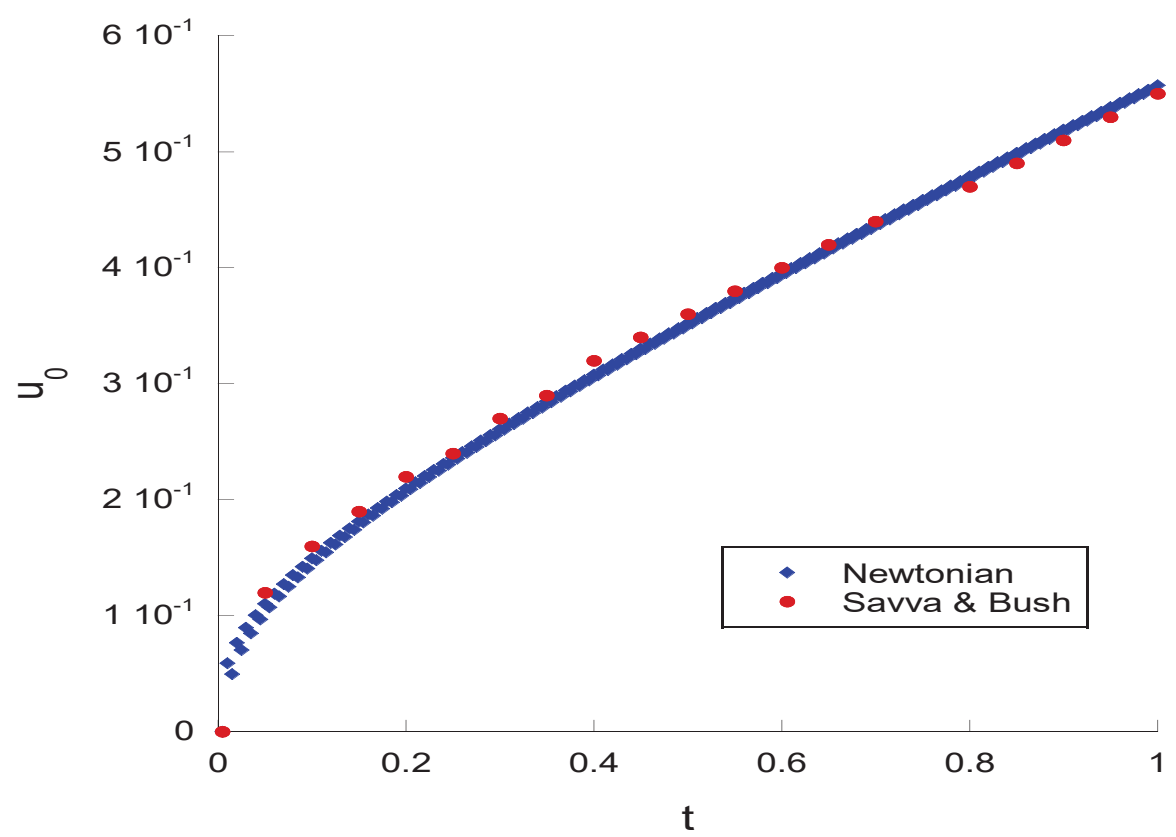

Figure 7.3: Validation of the numerical procedure for straight line retraction in Newtonian fluid with $O h=10$ by comparing with Savva and Bush results $[31]$

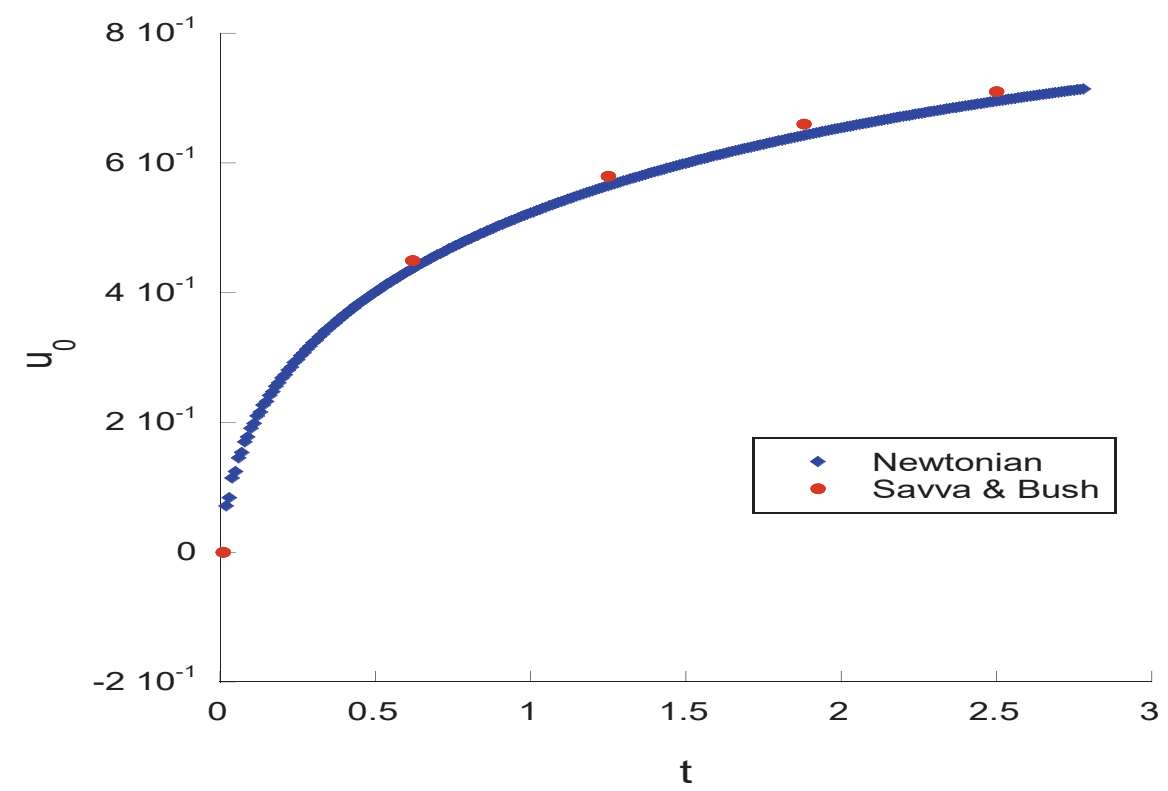

Figure 7.4: Validation of the numerical procedure for hole retraction in Newtonian fluid with $O h=10$ by comparing with Savva and Bush results [31] 


\section{8 \\ Results and discussions}

This capter presents the results of straight line and circular hole film retraction for Newtonian and non-Newtonian liquid sheets. Simillarly to rupture process, GNM and Oldroyd-B models are used to describe the non-Newtonian fluids. The analysis are performed for stationary initial condition for two viscosity regime: low $O h$ and high $O h$.

\section{1}

\section{High Oh regime}

Sheet retraction in high $O h$ regime was first investigated by Debrégeas et al. [65]. They performed an experimental study of the bursting process in films formed by a liquid with long chain polymer. Interesting features were observed in their studies: the absence of rim formation which was atributed to elastic stress and an exponential rate of retraction. Latter, numerical studies conducted by Savva and Bush [31] showed that high viscous stress induces the momentum diffusion away from the rim during the retraction process, making the film thickness to be more or less uniform. Thus viscosity by itself can explain the observation made by Debrégeas et al. [65].

Savva and Bush's studies left an open question about the role of the elastic stress in the retraction process of a high-viscous fluid. Therefore, here we look into this question by numerically investigating the effects of nonNewtonian properties on the retraction process of a liquid sheet in the high $O h$ regime. Results show the effects of non-Newtonian properties on the velocity retraction and on thickness shape. Analyses are performed for straight line and circular hole retraction in the early stage of the process.

\subsection{1}

\section{Oldroyd-B model}

This section presents the numerical results for film retraction of an Oldroyd-B fluid in high viscosity regime. The influence of non-Newtonian properties in the retraction dynamics is analyzed through the parameters $D e$ number and $\eta_{r}$. The Ohensorge number is kept fixed at $O h=10$ for all cases. 
Figures 8.1 and 8.2 show the temporal evolution of the tip velocity for straight line and circular hole retraction, respectively, taking into account different polymer-to-solvent viscosity ratio at $D e=1$. It is possible to observe that, in both cases, increasing $\eta_{r}$ the retraction process is decelerated, even in the early stage of the process. Hence, in high viscosity regime, elastic stresses act to hinder the retraction velocity in both geometry.

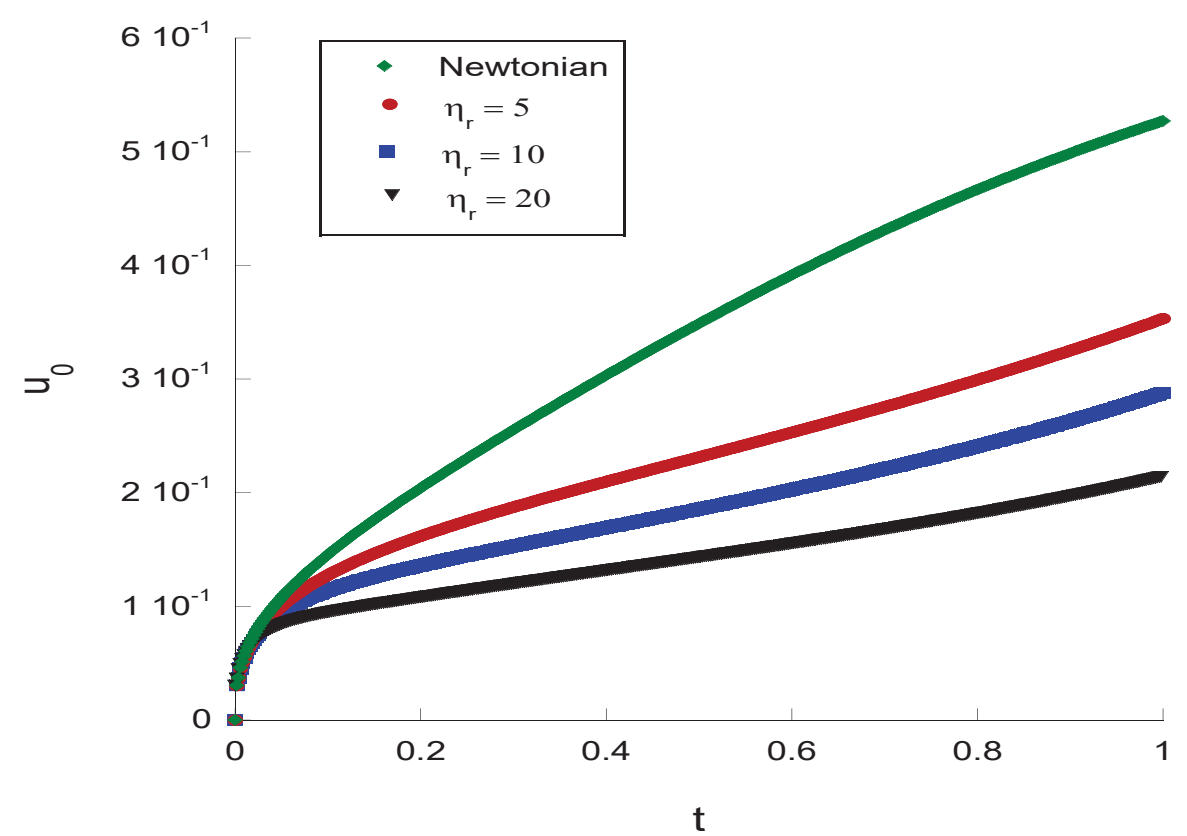

Figure 8.1: Tip velocity of straight line for Oldroyd-B model with $D e=1$ at $O h=10$ regime. 


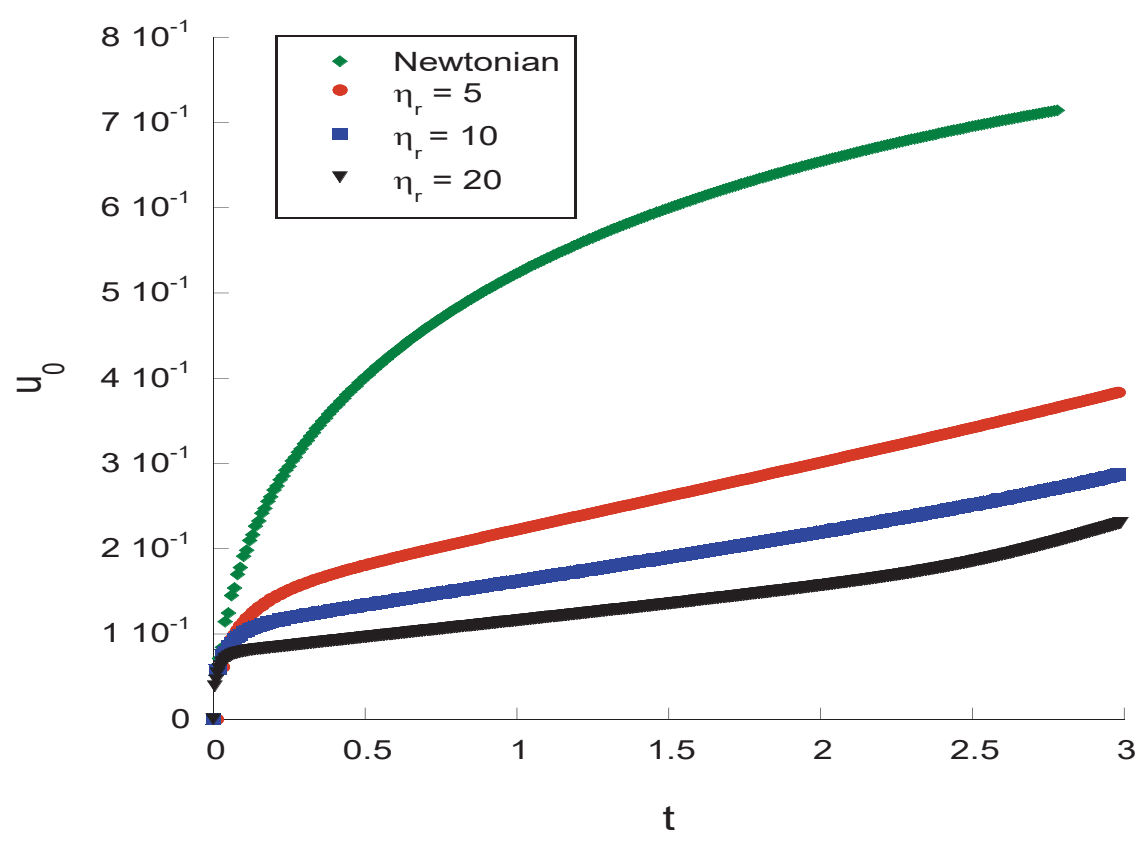

Figure 8.2: Tip velocity of a circular hole for Oldroyd-B model with $D e=1$ at $O h=10$ regime.

Debrégeas et al. [65] and Savva and Bush [31] have already discussed the absence of a rim formation in high $O h$ regime. However, an interesting feature can be observed in the retraction process of an Oldroyd-B fluid; the elastic stresses act in such away that film becomes flatter, as shown in Fig. 8.3.

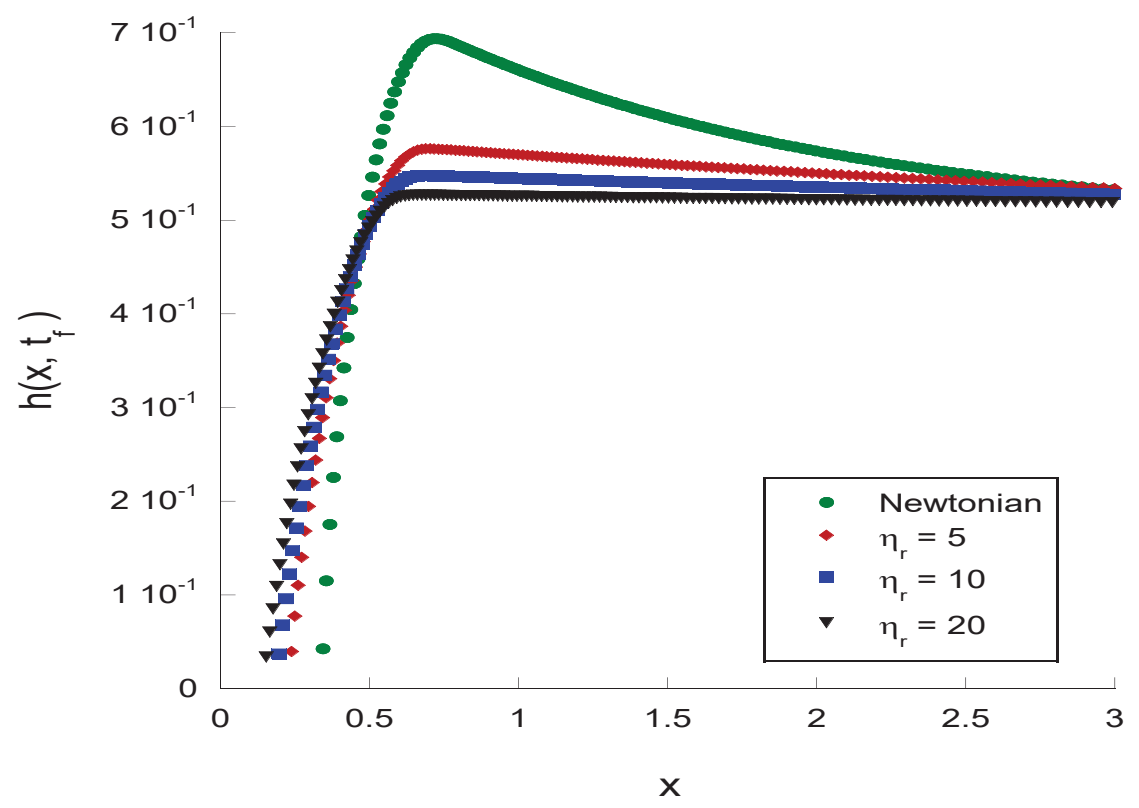

Figure 8.3: Thickness profile for Oldroyd-B model with $D e=1$ at $t=1$ in $O h=10$ regime. 
Net force analysis can provide interesting physical information about the retraction dynamics. Figure 8.4 presents all the difference forces along the film at $t=1$. They all decay to zero away fomr the tip. The axial viscoelastic force $\mathrm{T}_{x x}^{p}$, combined with viscous force act to hinder the straight line retraction driven by capillary force in a Oldroyd-B fluid.

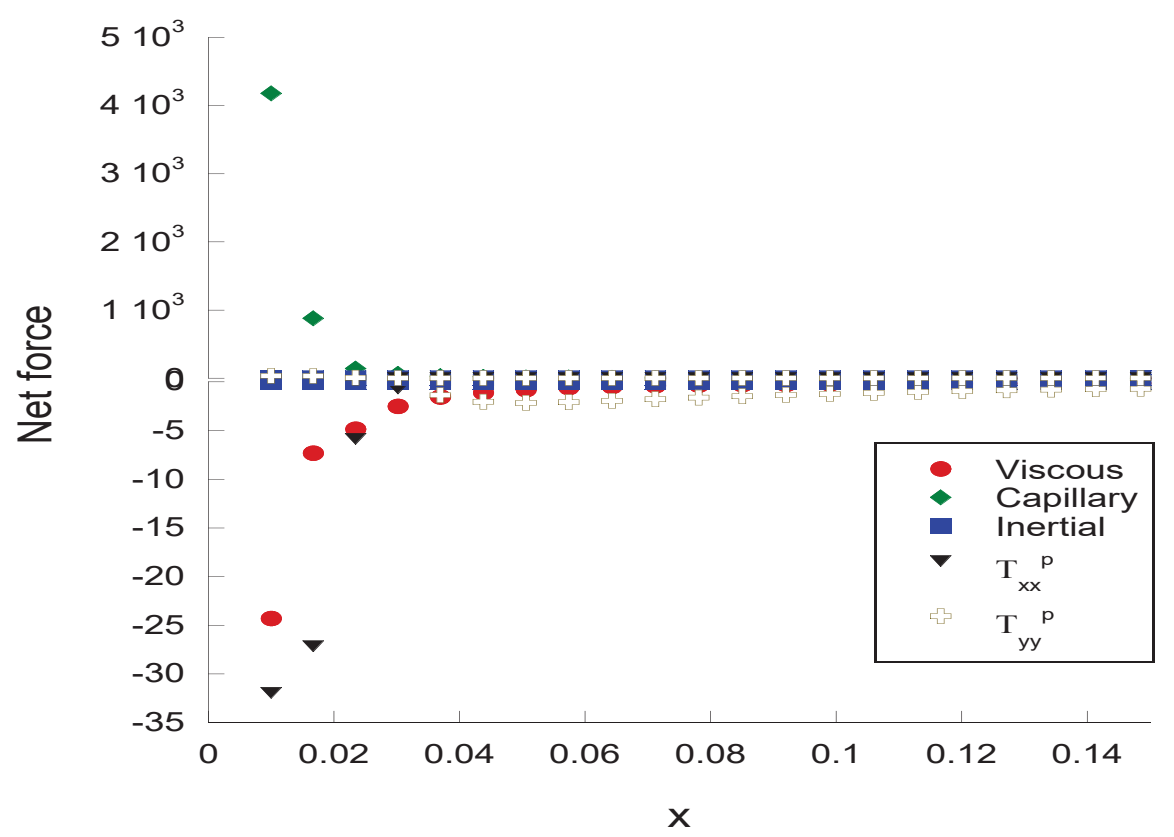

Figure 8.4: Net force for Oldroyd-B model with $D e=1$ and $\eta_{r}=10$ at $t=1$.

\subsection{2}

\section{Generalized Newtonian model}

As discussed earlier, GNM includes non-Newtonian effects only by allowing viscosity to change with rate-of-extension. Non-Newtonian parameters that control the flow behavior are also given by $D e$ and $\eta_{r}$, however, with different physical importance. Here, $D e$ in the non-dimensional parameter that represents the crossover from a region of zero-extensional-rate viscosity $\eta_{0}$ to a region of high-extensional-rate viscosity $\eta_{\infty}$. The ratio between those characteristic viscosities is given by $\eta_{r}=\eta_{\infty} / \eta_{0}$. By increasing $\eta_{r}$, the model allows a larger change in the viscosity of the liquid, and by increasing $D e$, this change occurs at a lower extensional rate.

Figure 8.5 presents an analysis of the influence of $\eta_{r}$ on the retraction velocity. It is possible to observe that the non-Newtonian properties of a GNM fluid have small effect on the retraction velocity, even for high $\eta_{r}$ and high $D e$ number. 


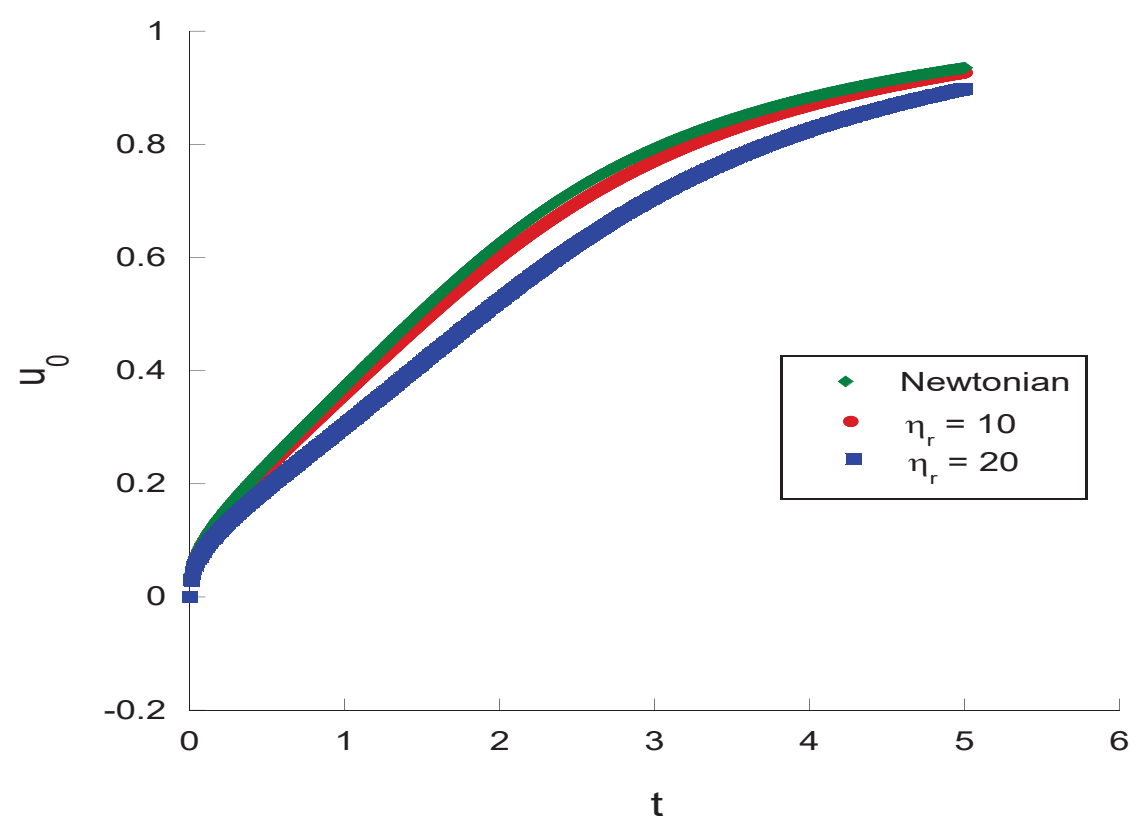

Figure 8.5: Tip velocity for GNM model with $D e=1$ at $O h=10$ regime.

The small influence of the GNM on the film retraction can be explained by the nature of non-Newtonian properties of the model; it is required a high rate-of-extension to induce a effective change in the fluid viscosity. However, as can be observed in Fig. 8.6, in the high $O h$ regime, the dynamics do not impose a high enough rate-of-extension, and consequently, $\eta_{r}$, which is set to be 10, does not reach $20 \%$ of that value, as shown in Fig.8.7.

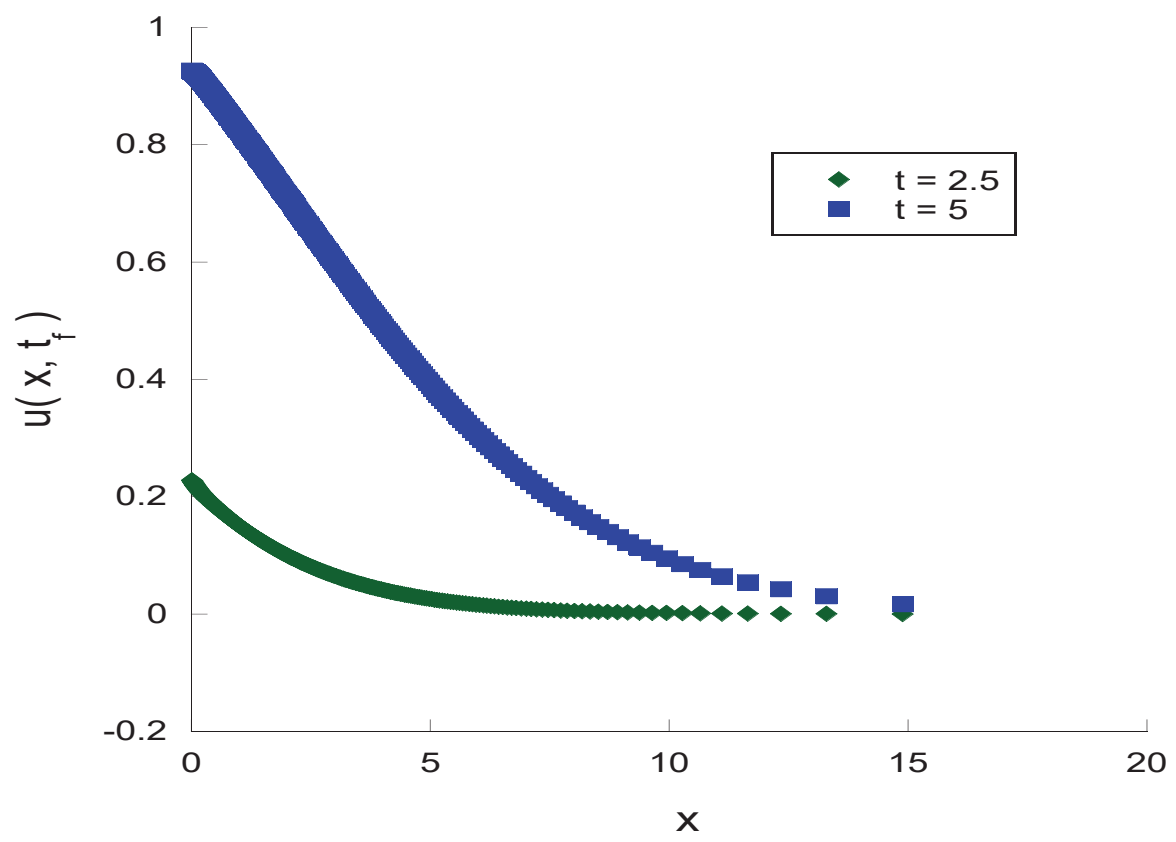

Figure 8.6: Longitudinal velocities profile for GNM model with $D e=1$ and $\eta_{r}=10$ at $O h=10$ regime. 


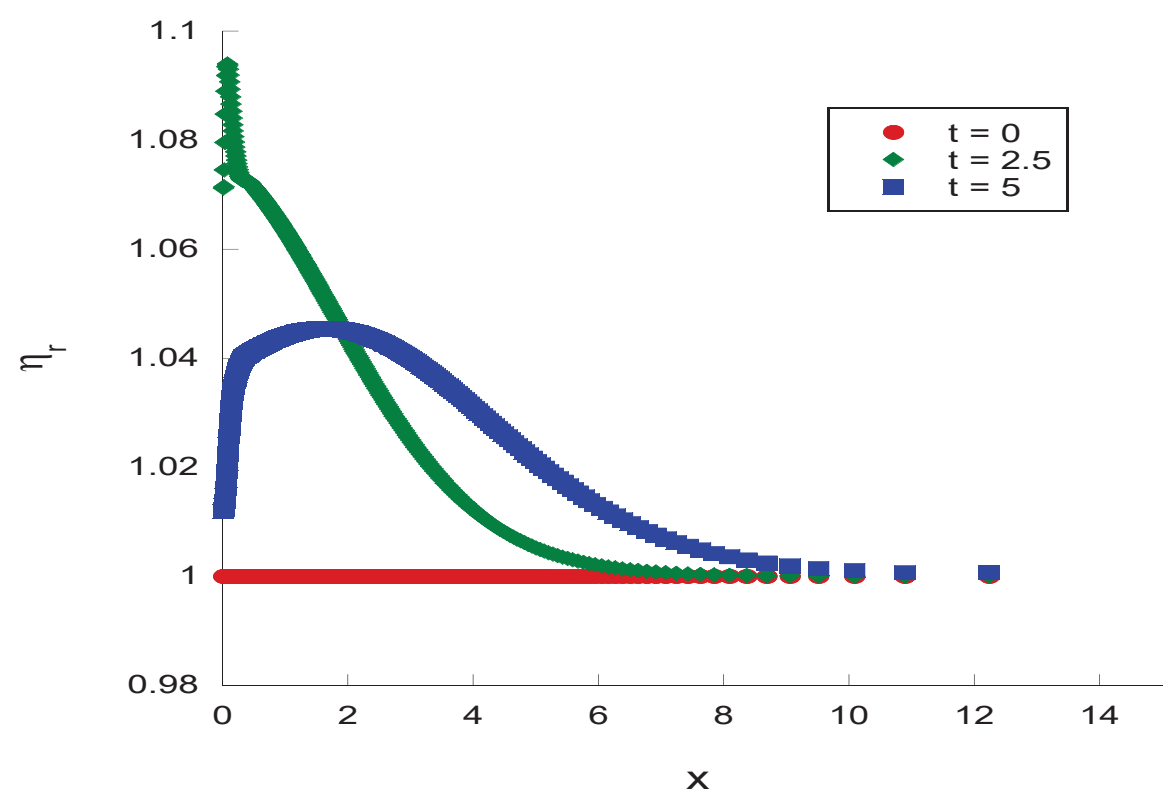

Figure 8.7: Viscosity profile for GNM model with $D e=1$ and $\eta_{r}=10$ at Oh $=10$ regime.

\section{2}

\section{Low Oh regime}

Low to moderate $\mathrm{Oh}$ regimes are present in most configurations of pratical importance such as those involving water-like fluid. The dissipation effect of viscosity is weaker and the motion becomes concentrated primarly near the edge of the film. A direct effect of the low viscous dissipation is the acceleration of the retraction process, as discussed earlier by Savva and Bush [31]. Another important aspect observed by Savva and Bush [31] was the formation of a rim during the retraction process, which was atributted to the inertia of the fluid ahead of the advancing edge. Also, Savva and Bush [31] observed that, for $O h<1$, capillary waves are generated ahead of the growing rim.

The analysis performed in Section 8.1 for high Oh regime of an OldroydB fluid showed that viscous and elastic forces work together to hinder the retraction process. It was observed that increasing the viscoelastic effects, the retraction velocity is substantialy reduced, even at the early stage of the process. In this section, the effect of non-Newtonian properties on the retraction process of a fluid with low viscous stress is analysed. 


\subsection{1}

\section{Oldroyd-B model}

Figures 8.8, 8.9 and 8.10 show that viscoelastic properties have an important role in hindering the circular hole velocity retraction for a fluid in the low viscosity regime. The influence of elastic properties seems to be more accentuated in a moderate $O h$ regime $(O h=1)$. It was observed a reduction of more than $50 \%$ of the edge velocity at $t=3$ for and Oldroyd-B fluid with $D e=1$ and $\eta_{r}=5(O h=1)$.

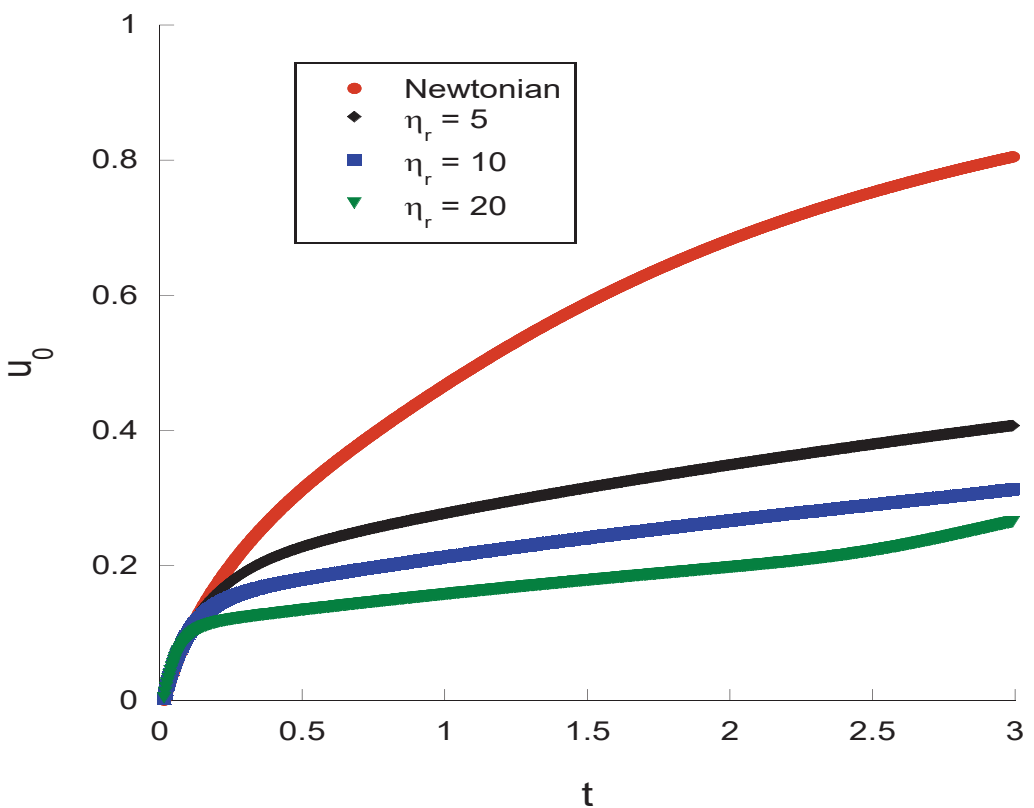

Figure 8.8: Tip velocity of a hole for Oldroyd-B model with $D e=1$ at $O h=1$ regime. 


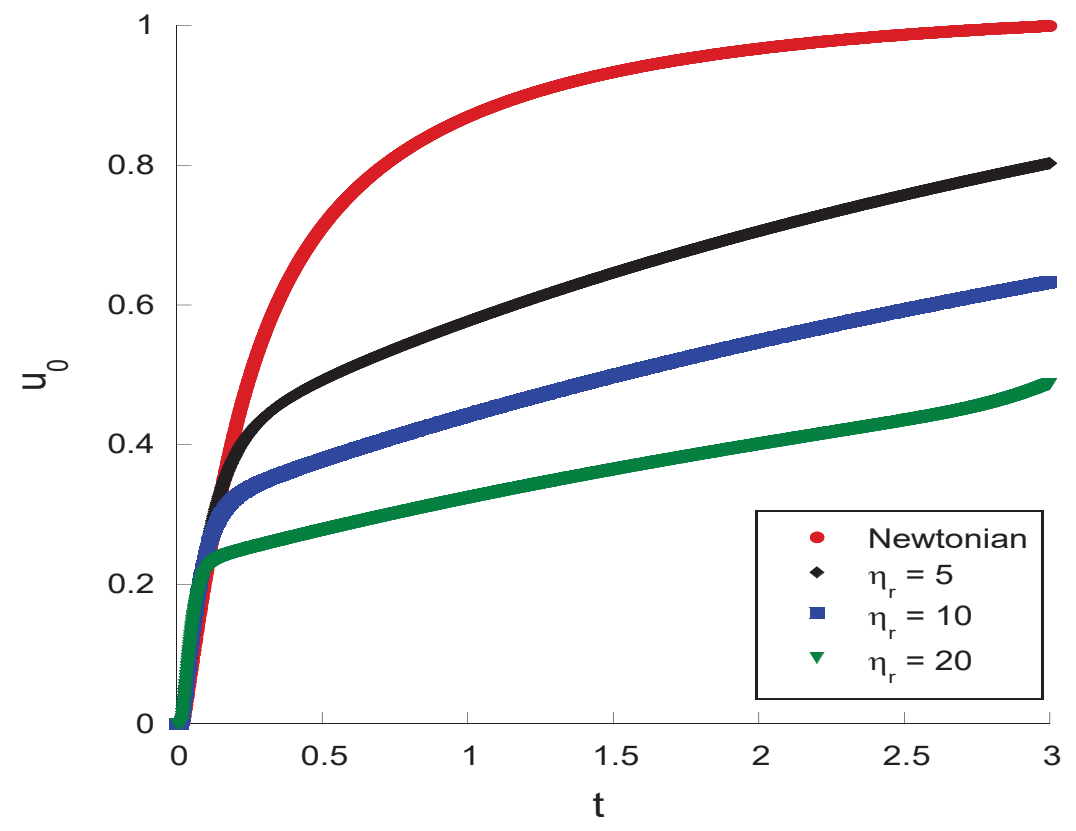

Figure 8.9: Tip velocity of a hole for Oldroyd-B model with $D e=1$ at $O h=0.5$ regime.

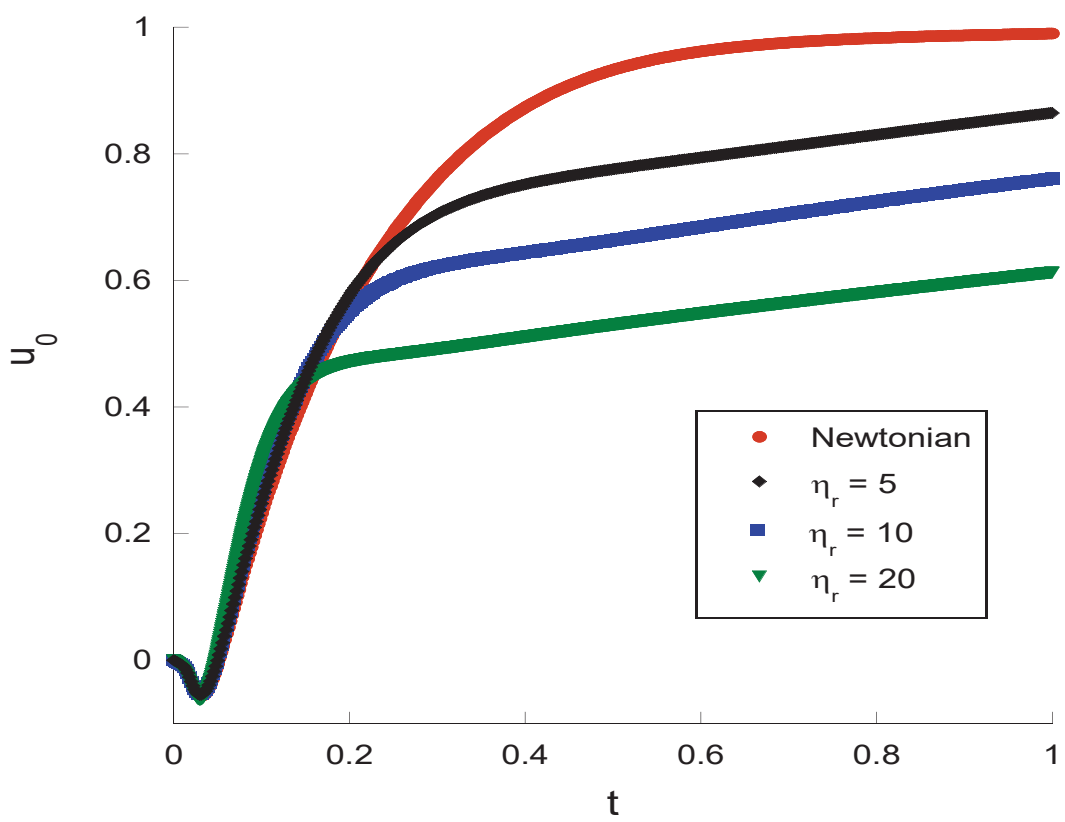

Figure 8.10: Tip velocity of a hole for Oldroyd-B model with $D e=1$ at $O h=0.1$ regime.

Another important result is related to the shape of the film. In a similar way to that observed in high viscosity films, Fig. 8.11 and 8.12 show that viscoelastic properties can prevent the formation of liquid rim in a low viscous fluid. For $O h=0.1$, Fig. 8.13, the absence of rim formation only was detected 
for a high elastic regime, $D e=1$ and $\eta_{r}=20$. However, it was observed the absence of capillary waves, at least in the early stages of retraction process, suggesting that viscoelastic effects can prevent or delay such kind of instabilities.

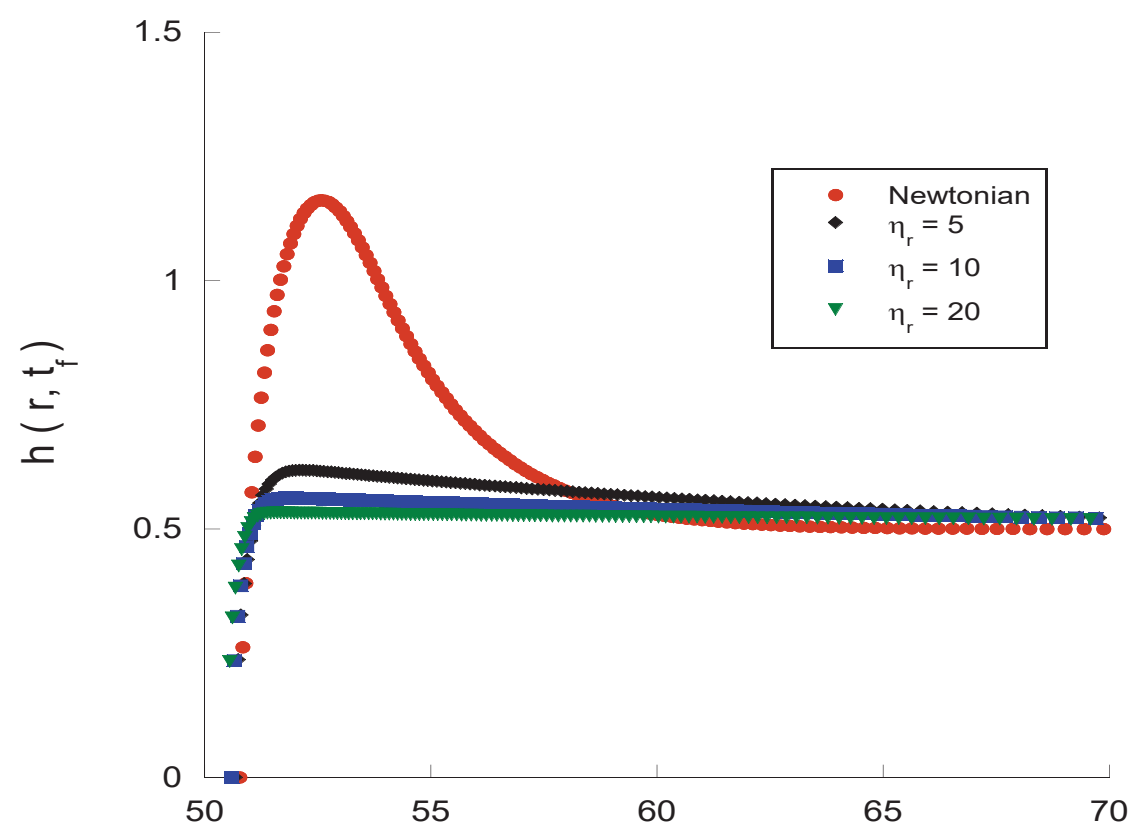

Figure 8.11: Thickness profile for Oldroyd-B model with $D e=1$ at $t=3$ in $O h=1$ regime.

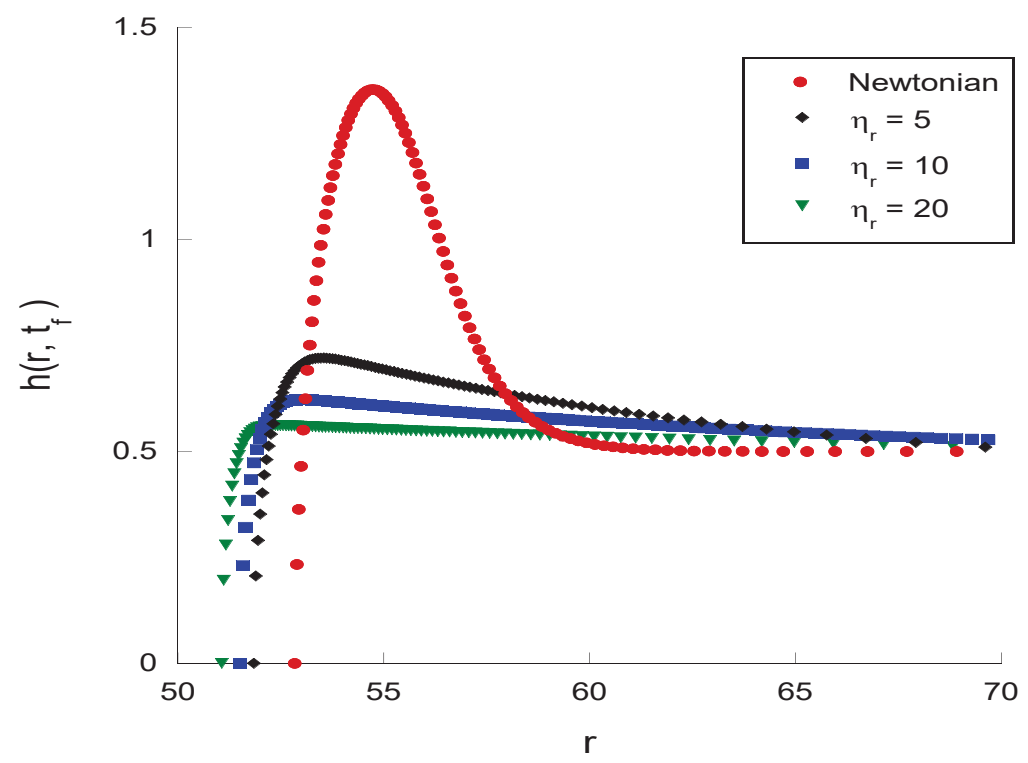

Figure 8.12: Thickness profile for Oldroyd-B model with $D e=1$ at $t=3$ in $O h=0.5$ regime. 


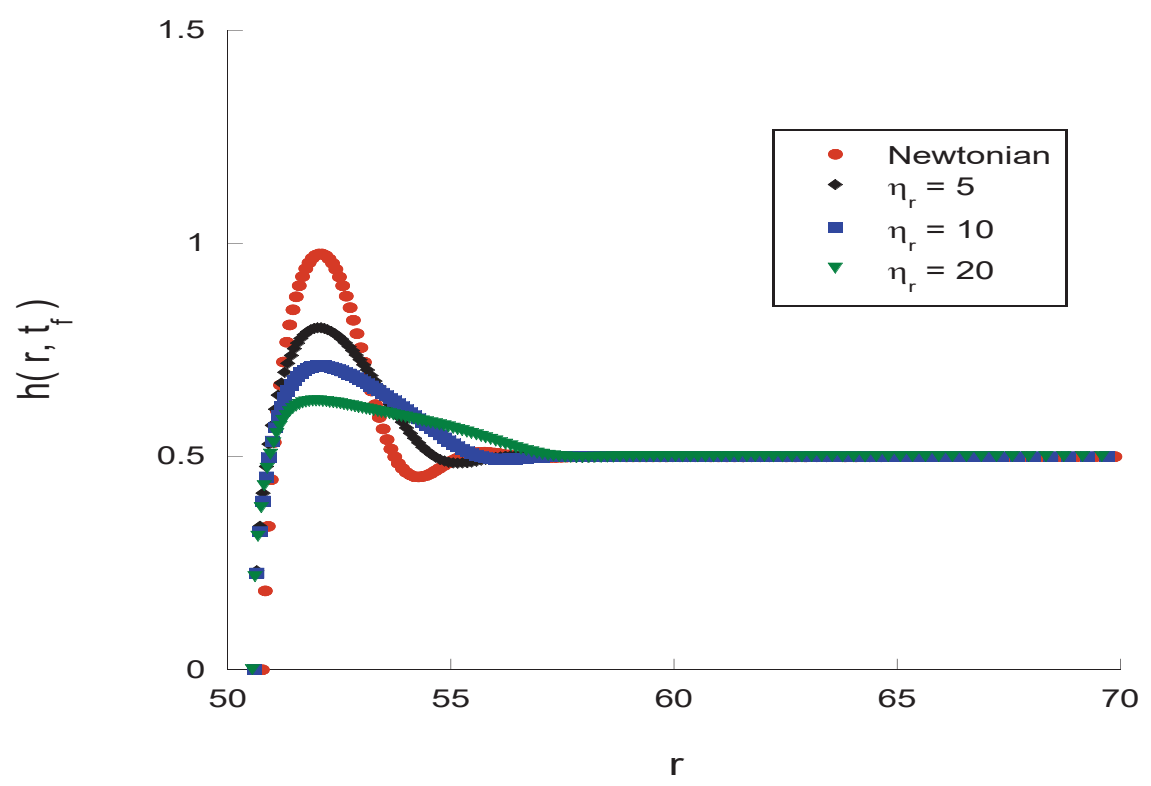

Figure 8.13: Thickness profile for Oldroyd-B model with $D e=1$ at $t=1$ in $O h=0.1$ regime.

The behavior of the film thickness profiles observed in Figs.8.11, 8.12 and 8.13 can be better understood by examining the axial velocity profile, presented in Figs. 8.14 and 8.15. The viscoelastic properties show an effect similar to the one observed at high viscosity regime, there is diffusion of momentum through the bulk of the film, which prevents the rim formation. Thus, by including viscoelastic properties the motion of a low viscosity fluid is no longer localized in the vicinity of the edge and begins to spread out through the radial direction. 


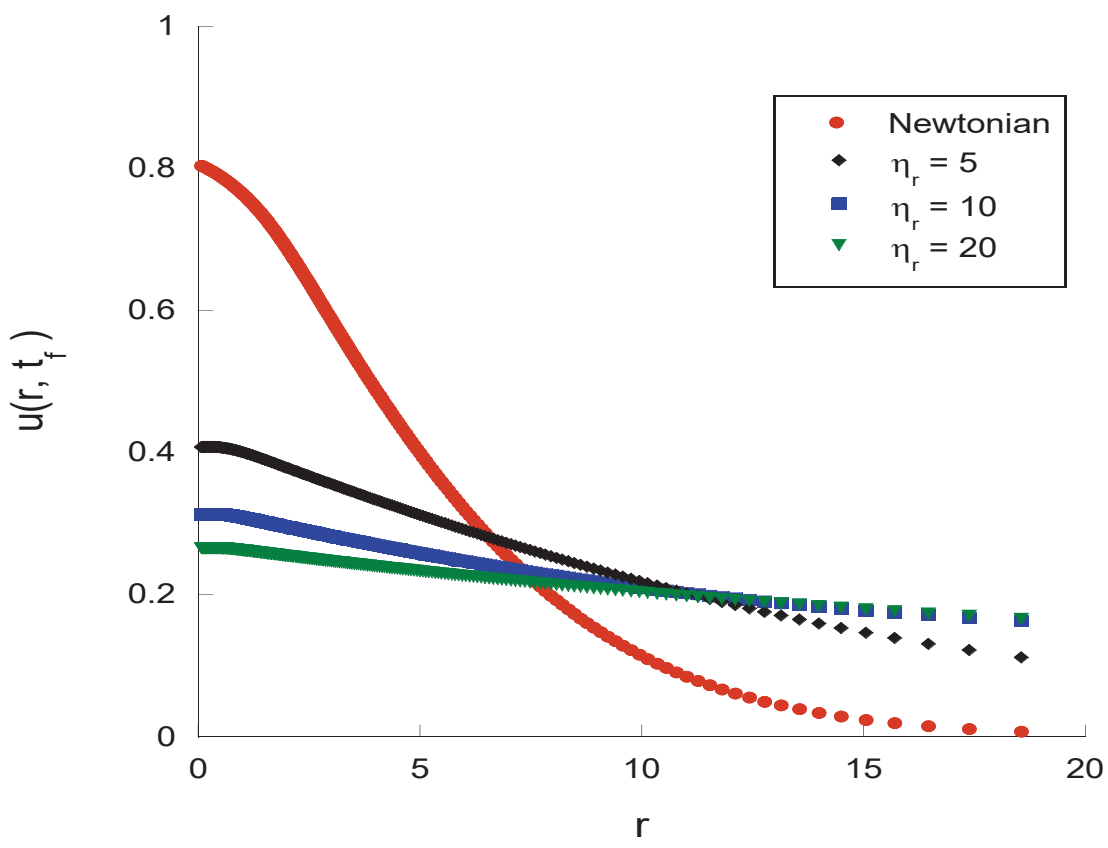

Figure 8.14: Thickness profile for Oldroyd-B model with $D e=1$ at $t=3$ in $O h=1$ regime.

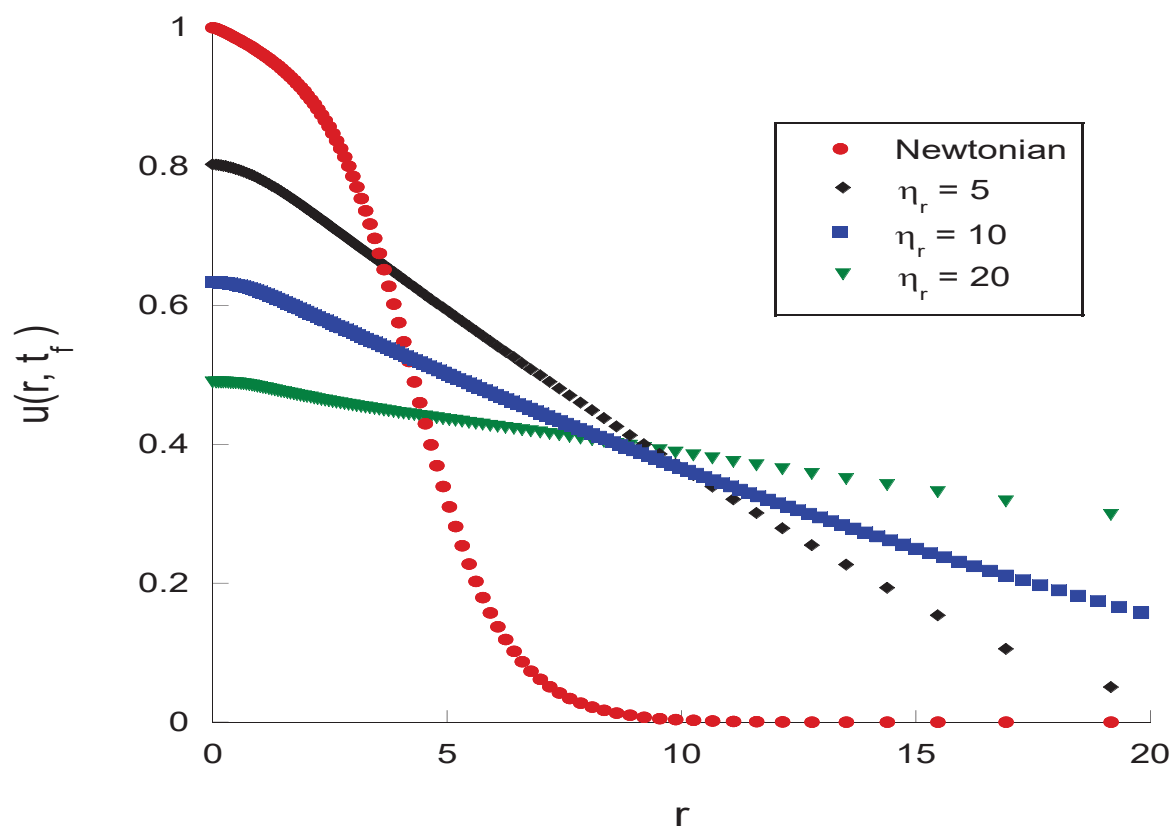

Figure 8.15: Thickness profile for Oldroyd-B model with $D e=1$ at $t=3$ in $O h=0.5$ regime. 


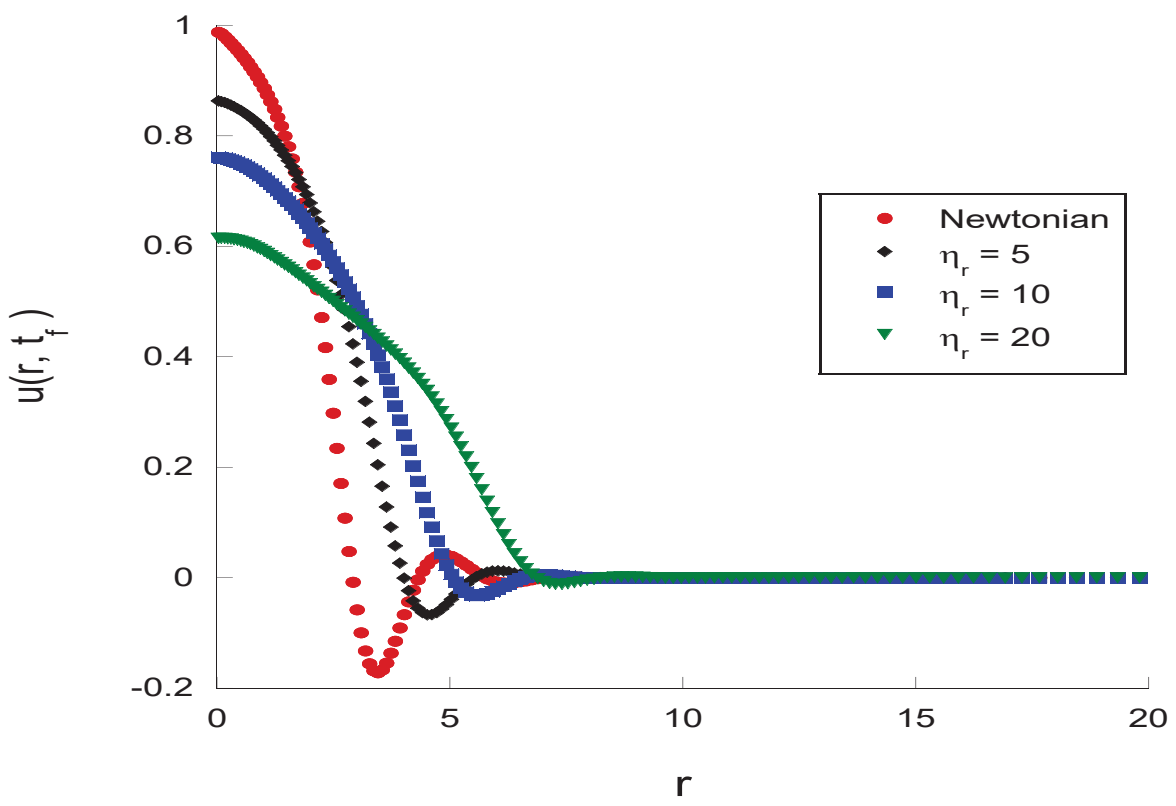

Figure 8.16: Thickness profile for Oldroyd-B model with $D e=1$ at $t=3$ in $O h=0.1$ regime. 


\section{9}

\section{Conclusions}

In this work, we presented an investigation of the entire breakup process of a viscoelastic thin liquid sheet. The process was divided into two separate steps: rupture and retraction. Viscoelastic effects were described by two models: the Oldroyd-B and generalized Newtonian model, that considers the viscosity a functions of the extensional rate.

The effect of viscoelastic forces on the rupture process of a liquid sheet was studied by solving the governing equations that describe the time evolution of planar and axisymmetric perturbations.

First, the stability criterion proposed by Erneux and Davis [25] for planar perturbation of a Newtonian liquid was extended to axisymmetric perturbations and Oldroyd-B liquids. The linear stability results suggest that an axisymmetric perturbation is more stable than a planar disturbance, due to the extra curvature that helps stabilize the liquid curtain. Predictions also show that the viscoelastic behavior has no effect on the critical condition (value of $S / \hat{A}$ ) below which the sheet is unstable.

The numerical solution of the evolution equations revealed that, when the van der Waals forces dominate and the sheet is unstable, the growth rate of axisymmetric disturbances is higher than that of planar perturbations, leading to shorter breakup time. The effect of the rheological behavior of the liquid was analyzed by varying the value of the Deborah number $D e$ and polymer-tosolvent viscosity ratio $\eta_{r}$. The results show that, despite not having any effect on the critical value of $S / \hat{A}$, the viscoelastic forces have a tremendous impact on the dynamics of the sheet rupture, slowing down the growth of the imposed perturbation and delaying the curtain pinch-off. These results may explain the experimental observation that curtain coating process can be stabilized by adding small amounts of high molecular weight polymers to the coating liquid.

For the retraction process, the non-Newtonian effects were studied through numerical solution of the evolution equations for two different viscosity regime; high $O h$ and low $O h$. In high $O h$ regime, earlier studies [65, 31] in Newtonian fluids showed that viscous force delays the retraction process due to the acceleration of a larger portion of the fluid. Another feature observed in this regime was the absence of the rim formation. Results of an Oldroyd- 
B fluid revealed that elastic effects further increase the features observed in high viscosity fluids. By including viscoelastic forces, the retraction process is decelerated and the thickness shape becomes flatter. Conversely, the analysis performed for a GNM fluid showed that the dynamic effects of the retraction process were not enough to activate non-Newtonian properties of that model, thus the non-Newtonian effects were barely perceptible.

In low Oh regime, numerical results of Oldroyd-B model showed important features associated with viscoelastic forces: for moderately low values of $O h$, i.e $O h=1$ and $O h=0.5$, elastic forces that arise na help to hinder the hole retraction process, avoiding the formation of the rim, in an effect similar to the observed in high viscosity fluid. An analysis of the axial velocity profile showed that elastic forces cause a momentum diffusion through the bulk of the liquid film. Additionally, results for $O h=0.1$, a viscosity regime marked by the presence of capillary waves in Newtonian films, shows the absence of ot it, revealing that elastic stresses avoid such instability that arise during the retraction process.

\section{1}

\section{Future works}

There are some open questions regarding the study of the dynamics of thin liquid sheets, especially because a large amount of fluids used in the industry is formed by particles and additives that can change its properties and affect the breakup dynamics. In this sense, an inclusion of a diffusion flux model to study the migration mechanism of suspended particles, as well as Marangoni stress associated to non-constant surface tension can be interesting for coating industry and could be explored for Newtonian an non-Newtonian liquid sheets.

From the studies presented in this work, a self-similarty analysis could be performed in order to propose a similarity solution for the rupture process of a viscoelastic fluid. In addition, the rupture dynamics of a double or a multiple layers of fluids, involving non-Newtonian and Newtonian fluid combination is still under-explored, though its large use by the industry, especially in coating applications.

Still, it is important to emphasize that there is a small number of experimental studies in the literature concerning breakup process of a nonNewtonian liquid sheet. In this sense, PIV methods and high-speed cameras could be used to evaluate the rupture and retraction process dynamics of viscoelastic sheets, ensuring the validity of numerical observation presented in this work. 


\section{Bibliography}

[1] VILLERMAUX, E.. Fragmentation. Annu. Rev. Fluid Mech., 39:419-446, 2007.

[2] WEINSTEIN, S. J.; RUSCHAK, K. J.. Coating flows. Annu. Rev. Fluid Mech., 36:29-53, 2004.

[8] RAYLEIGH, L.. On the instability of jets. Proceedings of the London mathematical society, 1(1):4-13, 1878.

[9] TAYLOR, G.; HOWARTH, L.. The dynamics of thin sheets of fluid. i. water bells. In: PROCEEDINGS OF THE ROYAL SOCIETY OF LONDON A: MATHEMATICAL, PHYSICAL AND ENGINEERING SCIENCES, volumen 253, p. 289-295. The Royal Society, 1959.

[10] TAYLOR, G.. The dynamics of thin sheets of fluid. ii. waves on fluid sheets. In: PROCEEDINGS OF THE ROYAL SOCIETY OF LONDON A: MATHEMATICAL, PHYSICAL AND ENGINEERING SCIENCES, volumen 253, p. 296-312. The Royal Society, 1959.

[11] TAYLOR, G.. The dynamics of thin sheets of fluid. iii. disintegration of fluid sheets. In: PROCEEDINGS OF THE ROYAL SOCIETY OF LONDON A: MATHEMATICAL, PHYSICAL AND ENGINEERING SCIENCES, volumen 253, p. 313-321. The Royal Society, 1959.

[12] BROWN, D. R.. A study of the behaviour of a thin sheet of moving liquid. Journal of Fluid Mechanics, 10(2):297-305, 1961.

[13] LIN, S.; W. LIAN, Z. ; J. CREIGHTON, B.. Absolute and convective instability of a liquid sheet. 220:673 - 689, 111990.

[14] FINNICUM, D. S.; WEINSTEIN, S. J. ; RUSCHAK, K. J.. The effect of applied pressure on the shape of a two-dimensional liquid curtain falling under the influence of gravity. Journal of Fluid Mechanics, 255:647-665, 1993.

[15] ROChE, J.; Le GRAND, N.; BRUnet, P.; LeBON, L. ; LIMAT, L.. Pertubations on a liquid curtain near break-up: Wakes and free edges. Physics of fluids, 18(8):082101, 2006. 
[16] DE LUCA, L.. Experimental investigation of the global instability of plane sheet flows. Journal of Fluid Mechanics, 399:355-376, 1999.

[17] KARIM, A. M.; SUSZYNSKI, W. J.; FRANCIS, L. F. ; CARVALHO, M. S.. Effect of viscosity on liquid curtain stability. AIChE Journal, DOI: 10.1002/aic.16015, 2017.

[18] BECERRA, M.; CARVALHO, M. S.. Stability of viscoelastic liquid curtain. Chemical Engineering and Processing: Process Intensification, 50(5):445-449, 2011.

[19] YANG, L.-J.; DU, M.-L.; FU, Q.-F.; TONG, M.-X. ; WANG, C.. Temporal instability of a power-law planar liquid sheet. Journal of Propulsion and Power, 31(1):286-293, 2015.

[20] LIFSHITZ, E.. The theory of molecular attractive forces between solids. Sov. Phys. JETP, p. 73-83, 1956.

[21] DZYALOSHINSKII, I.; PITAEVSKII, L.. Vanderwaals forces in an inhomogeneous dielectric. Sov. Phys. JETP, 9(6):1282-1287, 1959.

[22] DZYALOSHINSKII, I.; LIFSHITZ, E. ; PITAEVSKII, L.. Van der waals forces in liquid films. Perspectives in Theoretical Physics: The Collected Papers of $E \backslash M \backslash$ Lifshitz, p. 425, 2012.

[23] VRIJ, A.. Possible mechanism for the spontaneous rupture of thin, free liquid films. Discussions of the Faraday Society, 42:23-33, 1966.

[24] PRÉVOST, M.; GALLEZ, D.. Nonlinear rupture of thin free liquid films. The Journal of chemical physics, 84(7):4043-4048, 1986.

[25] ERNEUX, T.; DAVIS, S. H.. Nonlinear rupture of free films. Physics of Fluids A: Fluid Dynamics, (5):1117-1122, 1993.

[26] BATCHELOR, G. K.. An introduction to fluid dynamics. Cambridge university press, 2000.

[27] IDA, M.; MIKSIS, M.. Thin film rupture. Applied Mathematics Letters, 9(3):35-40, 1996.

[28] FERZIGER, J. H.; PERIC, M.. Computational methods for fluid dynamics. Springer Science \& Business Media, 2012.

[31] SAVVA, N.; BUSH, J. W.. Viscous sheet retraction. Journal of Fluid Mechanics, 626:211-240, 2009. 
[39] BIRD, R. B.; ARMSTRONG, R. C.; HASSAGER, O. ; CURTISS, C. F.. Dynamics of polymeric liquids, volumen 1. Wiley New York, 1977.

[41] SÜNDERHAUF, G.; RASZILLIER, H. ; DURST, F.. The retraction of the edge of a planar liquid sheet. Physics of Fluids, 14(1):198-208, 2002.

[42] EGGERS, J.; DUPONT, T. F.. Drop formation in a one-dimensional approximation of the navier-stokes equation. Journal of fluid mechanics, 262:205-221, 1994.

[43] VAYNBLAT, D.; LISTER, J. R. ; WITELSKI, T. P.. Rupture of thin viscous films by van der waals forces: Evolution and self-similarity. Physics of Fluids, 13(5):1130-1140, 2001.

[44] THETE, S. S.; ANTHONY, C.; BASARAN, O. A. ; DOSHI, P.. Self-similar rupture of thin free films of power-law fluids. Physical Review E, 92(2):023014, 2015.

[46] LARSON, R. G.. Constitutive Equations for Polymer Melts and Solutions: Butterworths Series in Chemical Engineering. Butterworth-Heinemann, 2013.

[47] MALI, M.; GOYAL, N. ; TRIVEDI, H.. Geometric methods in the theory of ordinary differential equations: A review.

[48] MACOSKO, C. W.. Rheology: principles, measurements, and applications. Wiley-vch, 1994.

[49] MORRISON, F. A.. Understanding rheology. Topics in Chemical Engineering, 2001.

[51] VAYNBLAT, D.; LISTER, J. R. ; WITELSKI, T. P.. Symmetry and self-similarity in rupture and pinchoff: a geometric bifurcation. European Journal of Applied Mathematics, 12(3):209-232, 2001.

[52] GRATTON, J.. Similarity and self similarity in fluid dynamics. Fundamentals of Cosmic Physics, 15:1-106, 1991.

[54] DE WIT, A.; GALLEZ, D. ; CHRISTOV, C.. Nonlinear evolution equations for thin liquid films with insoluble surfactants. Physics of fluids, 6(10):3256-3266, 1994.

[55] SHARMA, A.; KISHORE, C.; SALANIWAL, S. ; RUCKENSTEIN, E.. Nonlinear stability and rupture of ultrathin free films. Physics of Fluids, 7(8):1832-1840, 1995. 
[56] SHUGAI, G.; YAKUBENKO, P.. Spatio-temporal instability in free ultra-thin films. European Journal of Mechanics-B/Fluids, 17(3):371-384, 1998.

[57] MATAR, O.; SPELT, P.. Dynamics of thin free films with reaction-driven density and viscosity variations. Physics of Fluids, 17(12):122102, 2005.

[58] THETE, S. S.; ANTHONY, C.; DOSHI, P.; HARRIS, M. T. ; BASARAN, $O$. A.. Self-similarity and scaling transitions during rupture of thin free films of newtonian fluids. Physics of Fluids, 28(9):092101, 2016.

[60] DUPRÉ, A.; DUPRÉ, P.. Théorie mécanique de la chaleur. GauthierVillars, 1869.

[62] CULICK, F.. Comments on a ruptured soap film. Journal of applied physics, 31(6):1128-1129, 1960.

[63] MCENTEE, W. R.; MYSELS, K. J.. Bursting of soap films. i. an experimental study. The Journal of Physical Chemistry, 73(9):30183028, 1969.

[65] DEBRÉGEAS, G.; MARTIN, P. ; BROCHARD-WYART, F.. Viscous bursting of suspended films. Physical review letters, 75(21):3886, 1995.

[66] BRENNER, M. P.; GUEYFFIER, D.. On the bursting of viscous films. Physics of Fluids, 11(3):737-739, 1999.

[67] SONG, M.; TRYGGVASON, G.. The formation of thick borders on an initially stationary fluid sheet. Physics of Fluids, 11(9):2487-2493, 1999.

[68] VILLONE, M. M.; D'AVINO, G.; DI MAIO, E.; HULSEN, M. A. ; MAFFETTONE, P. L.. Modeling and simulation of viscoelastic film retraction. Journal of Non-Newtonian Fluid Mechanics, 249:26-35, 2017.

[69] MOROZOV, A.; SPAGNOLIE, S. E.. Introduction to complex fluids. In: COMPLEX FLUIDS IN BIOLOGICAL SYSTEMS, p. 3-52. Springer, 2015.

[70] GORDILlO, L.; AGBAGLAH, G.; DUCHEMIN, L. ; JOSSERAND, C.. Asymptotic behavior of a retracting two-dimensional fluid sheet. Physics of Fluids, 23(12):122101, 2011. 
A

\section{Published paper}

BAZZI, M. S.; CARVALHO M. S, Effect of viscoelastic forces on liquid sheet rupture process, submitted to Journal of Non Newtonian Fluid Mechanics, 2018. 